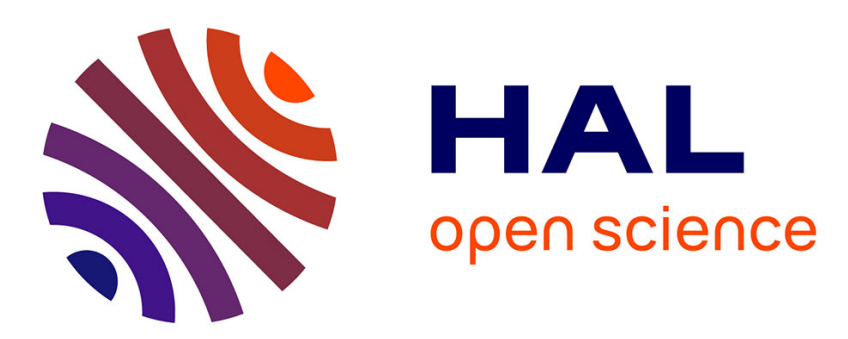

\title{
(SI) Band Gap Tuning in Nanodiamonds: First Principle Computational Studies
}

Peter R Schreiner, Andrey A Fokin

\section{To cite this version:}

Peter R Schreiner, Andrey A Fokin. (SI) Band Gap Tuning in Nanodiamonds: First Principle Computational Studies. Molecular Physics, 2009, 107 (08-12), pp.823-830. 10.1080/00268970802649625. hal-00513243

\section{HAL Id: hal-00513243 \\ https://hal.science/hal-00513243}

Submitted on 1 Sep 2010

HAL is a multi-disciplinary open access archive for the deposit and dissemination of scientific research documents, whether they are published or not. The documents may come from teaching and research institutions in France or abroad, or from public or private research centers.
L'archive ouverte pluridisciplinaire HAL, est destinée au dépôt et à la diffusion de documents scientifiques de niveau recherche, publiés ou non, émanant des établissements d'enseignement et de recherche français ou étrangers, des laboratoires publics ou privés. 


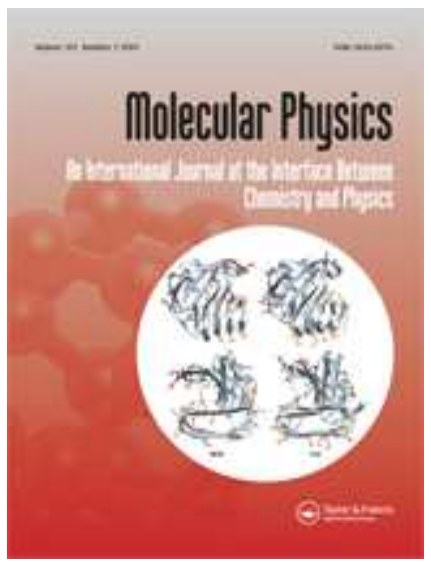

\section{(SI) Band Gap Tuning in Nanodiamonds: First Principle Computational Studies}

\begin{tabular}{|r|l|}
\hline Journal: & Molecular Physics \\
\hline Manuscript ID: & TMPH-2008-0322.R1 \\
\hline Manuscript Type: & Special Issue Paper - Fritz Schaefer \\
\hline Date Submitted by the \\
Author: & 23-Nov-2008 \\
\hline Complete List of Authors: & $\begin{array}{l}\text { Schreiner, Peter; Justus-Liebig-University, Institute of Organic } \\
\text { Chemistry } \\
\text { Fokin, Andrey; Kiev Polytechnic Institute, Department of Organic } \\
\text { Chemistry }\end{array}$ \\
\hline Keywords: & nanodiamonds, density functional theory, band gap, doping \\
\hline
\end{tabular}

\section{$\checkmark$ ScholaroNE" \\ Manuscript Central}




\title{
Band Gap Tuning in Nanodiamonds: First Principle Computational Studies $^{\$}$
}

\author{
Andrey A. Fokin ${ }^{\mathrm{a}, \mathrm{b}^{*}}$ and Peter R. Schreiner ${ }^{\mathrm{b} *}$ \\ ${ }^{a}$ Department of Organic Chemistry, Kiev Polytechnic Institute, pr. Pobedy 37, 03056 Kiev, Ukraine and ${ }^{b}$ Institute of Organic \\ Chemistry, Justus-Liebig University, Heinrich-Buff-Ring 58, D-35392 Giessen, Germany
}

(Received...)

\begin{abstract}
We present a density functional theory study on changes in band gap effects of nanodiamonds (hydrogen terminated diamond-like molecules, diamondoids) depending on size, shape, and the incorporation of heteroatom functionalities. Strong quantum confinement effects were identified at particle sizes from 0.5 to at least $2 \mathrm{~nm}$, when the band gaps of these nanodiamonds are reduced to $6.7 \mathrm{eV}$. Octahedral and tetrahedral nanodiamonds show the same trends in band gap narrowing, and it is the dimension rather than the shape/morphology of the nanodiamonds that alters the band gaps. Band gap tuning through external (by $\mathrm{C}-\mathrm{H}$ bond substitution) or internal (by replacing $\mathrm{CH}$ or $\mathrm{CH}_{2}$ moieties) doping is non-additive for the same dopant. Push-pull doping, with electron donating and electron withdrawing groups is most effective and reduces the band gaps of diamondoids to that of bulk diamond. Further reductions down to $1-2 \mathrm{eV}$ are conceivable with charged external substituents. The combination of increasing the size of the nanodiamond and push-pull doping are likely to make these materials highly valuable for semiconductor applications.
\end{abstract}

Keywords: Diamondoids; DFT methods; electronic gaps; nanodiamonds

\section{Introduction}

Nanodiamonds are nm-sized single-molecule diamond-like hydrocarbons (so-called diamondoids) that nicely close the gap between chemistry, physics, and the material sciences (for the first representatives of this series of molecules see Scheme 1). ${ }^{[1,2]}$ They represent the long underrated world of $\mathrm{sp}^{3}$ carbon materials that have complementary properties to the nowadays ubiquitous fullerenes and carbon nanotubes. ${ }^{[3]}$ The physics community uses the term "nanodiamond" in singular form for the constituents of a variety of systems ranging from interstellar dusts and meteorites, carbonaceous residues of detonations to diamond-like films. $^{[4]}$ The combination of strength, hardness, semiconductivity (upon doping), electron field emission, ${ }^{[5]}$ and unique optical properties make diamond-like materials particularly promising for discovering unusual properties at the borderline between chemistry and physics and for designing novel materials.

For a long time the chemistry of diamondoids was associated only with adamantane $\mathrm{C}_{10} \mathrm{H}_{16}$

\footnotetext{
\$Functionalized Nanodiamonds, part 16. For part 15, see: C. Sinkel, S. Agarwal, N. A. Fokina, P. R. Schreiner Macromol. Chem. Phys. 2008, 209, submitted

*Corresponding authors: aaf@xtf.ntu-kpi.kiev.ua and prs@org.chemie.uni-giessen.de
} 
(1),${ }^{[6]}$ whose derivatives have found an incredible number of highly practical applications; ${ }^{[7]}$ 1, diamantane $(2),{ }^{[8]}$ triamantane $(3),{ }^{[9]}$ and [121]tetramantane $(\mathbf{4})^{[10]}$ have also been synthesized chemically. Despite some recent experimental progress, ${ }^{[11-13]}$ much of the chemistry of higher diamondoids (those with isomeric structure, i.e., starting with tetramantane, 4) remains largely unexplored as sizeable quantities of these hydrocarbons became readily available from natural sources only recently. ${ }^{[14]}$ Diamondoids were found in substantial amounts in petroleum cuts ${ }^{[15]}$ and natural gas condensates. ${ }^{[16]}$ The big advantage of diamondoids is that they are, in contrast to detonation and CVD nanodiamond, "knowable," i.e., they are physically and chemically well characterized and are all structurally homogeneous. ${ }^{[1]}$ Chemical purity and homogeneity is a conditione sine qua non for using carbon materials for applications and as a test bed for $\mathrm{sp}^{3}$ nanomaterials.

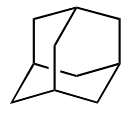

1, Adamantane

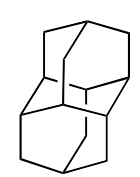

2, Diamantane

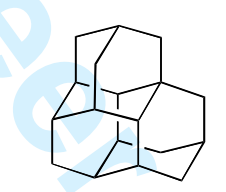

3, Triamantane

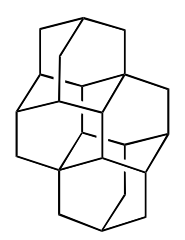

4a, [121]Tetramantane,

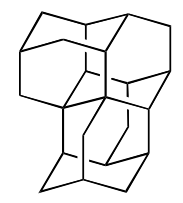

$\pm 4 \mathbf{b}$, [123]Tetramantane

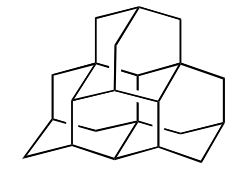

4c, [1(2)3]Tetramantane

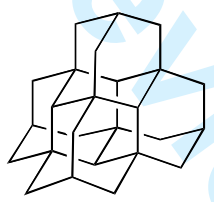

5, [1(2,3)4]Pentamantane

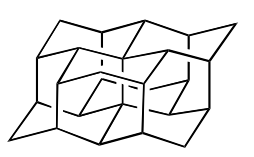

6. Cyclohexamantane

Scheme 1. First members in the series of diamondoid hydrocarbons (nanodiamonds) with numbering of carbon atoms.

A particular promising scientific road is the ability to selectively replace diamondoid $\mathrm{C}-\mathrm{H}$ bonds with a large variety of substituents. ${ }^{[11,12,17]}$ Moreover, it is also likely to be possible to substitute methylene groups $\left(-\mathrm{CH}_{2}-\right)$ with heteroatom functionalities $(\mathrm{O}, \mathrm{S}, \mathrm{NH}$, etc, $)$, which constitutes a rational synthetic approach to selectively doping diamond materials. As doped diamond shows unique properties such as pronounced semiconductivity, fluorescence shifts, deep coloration, ${ }^{[18]}$ it would be highly desirable to prepare materials with such properties in a predictable and reproducible fashion. At the same time, computational assessments ${ }^{[19]}$ of the properties to be expected will provide valuable guidelines as to which dopants and which diamondoid types are most promising for particular (mostly seminconductor) applications. 
Herein we examine computationally three different approaches for band gap tuning of diamondoids, as the band gap of such materials (including undoped bulk diamond) is just too high to be useful for semiconductor applications. The first approach is based on the enlargement and changing of the shapes/morphologies of the particles themselves to probe quantum confinement effects. ${ }^{[20,21]}$ Second, we examine the effect of "external doping" based on $\mathrm{C}-\mathrm{H}$ bond substitutions with various functional groups. Finally, we present an analysis of "interstitial" or internal doping by incorporating heteroatom functionalities of one or more $\mathrm{CH}$ or $\mathrm{CH}_{2}$ fragments.

\section{Computational Methods}

All computations were performed with the GAUSSIAN03 program suite ${ }^{[22]}$ employing analytical first and second energy derivatives. Harmonic vibrational frequencies were computed to characterize stationary points. We used the $\mathrm{B}^{2} \mathrm{LYP}^{[23]}$ functional for comparison with earlier results and the $\mathrm{B} 3 \mathrm{PW} 91^{[24]}$ functional because it is more trustworthy for large molecules than many other popular DFT methods. ${ }^{[25]}$ We utilized 6-31G(d) and 6-31G(d,p) basis sets for all computations. ${ }^{[26]}$

\section{Results and Discussion}

Size/shape dependence of the band gap. Previous attempts to examine size effects of diamond nanoclusters on the band gap are rather controversial as the results are sensitive to the theoretical method employed. GGA and TDLDA computations ${ }^{[27]}$ give band gaps for large diamond clusters that are lower than for bulk diamond $(5.5 \mathrm{eV})$ and quantum confinement effects were considered negligible for diamond particles larger than $1 \mathrm{~nm}$. Diffusion Monte-Carlo computations ${ }^{[28]}$ predict larger band gaps and confirmed the size dependence for quantum confinement. However, these results are not supported by tightbinding simulations ${ }^{[29]}$ for which the effects disappear for particles larger than $2.5 \mathrm{~nm}$. Our conclusions is that some computational approaches simply are not able to reproduce quantitatively the orbital energies in nanodiamonds and are therefore unsuitable to study these materials. This conclusion is confirmed by the enormous discrepancies in reported band gap values ranging from $0.74 \mathrm{eV}$ to $9.3 \mathrm{eV}$ for adamantane (for a detailed discussion see ref. ${ }^{[30]}$ ). In general, periodic plane-wave DFT approaches underestimate the band gaps in nanodiamond materials substantially. For instance, recent plane-wave pseudopotential computations reveal a $7.9 \mathrm{eV}$ band gap in [121]tetramantane, ${ }^{[31]}$ whereas our computed B3PW91/6-31G(d,p) value is $8.6 \mathrm{eV}$ (we will argue below that this is likely to be much closer 
to experiment). A new study on the direct band gaps of the cyrstals of lower diamondoids utilizing plane waves and periodic boundary conditions also notes that such approaches (here with GGA and LDA combinations for the correlation and exchange potentials) underestimate the true band gaps of these materials. ${ }^{[32]}$ However, these periodic methods qualitatively confirmed $^{[28]}$ the very important experimental observation that the unoccupied states in carbon nanoparticles, in contrast to their silicon or germanium counterparts, do not show quantum confinement effects. ${ }^{[20]}$

The first nonperiodic, i. e., molecular DFT (B3LYP) computations on small ${ }^{[33]}$ as well as large $\mathrm{e}^{[34]}$ diamond nanoclusters including vibrational frequency computations covered nanocarbon particles with up to 286 carbon atoms. Despite the use of moderately sized basis sets [6-21G for $\mathrm{C}$ and 6-31G(d,p) for $\mathrm{H}]^{[34]}$ reasonable results were obtained: The band gap of bulk diamond was not reached even for the $3 \mathrm{~nm}$ particle $\mathrm{C}_{969} \mathrm{H}_{324}$ that has a band gap of 6.6 eV. However, only octahedral diamondoids where studied leaving the question of the influence of the shape (morphology) of the particle on the band gap unanswered.

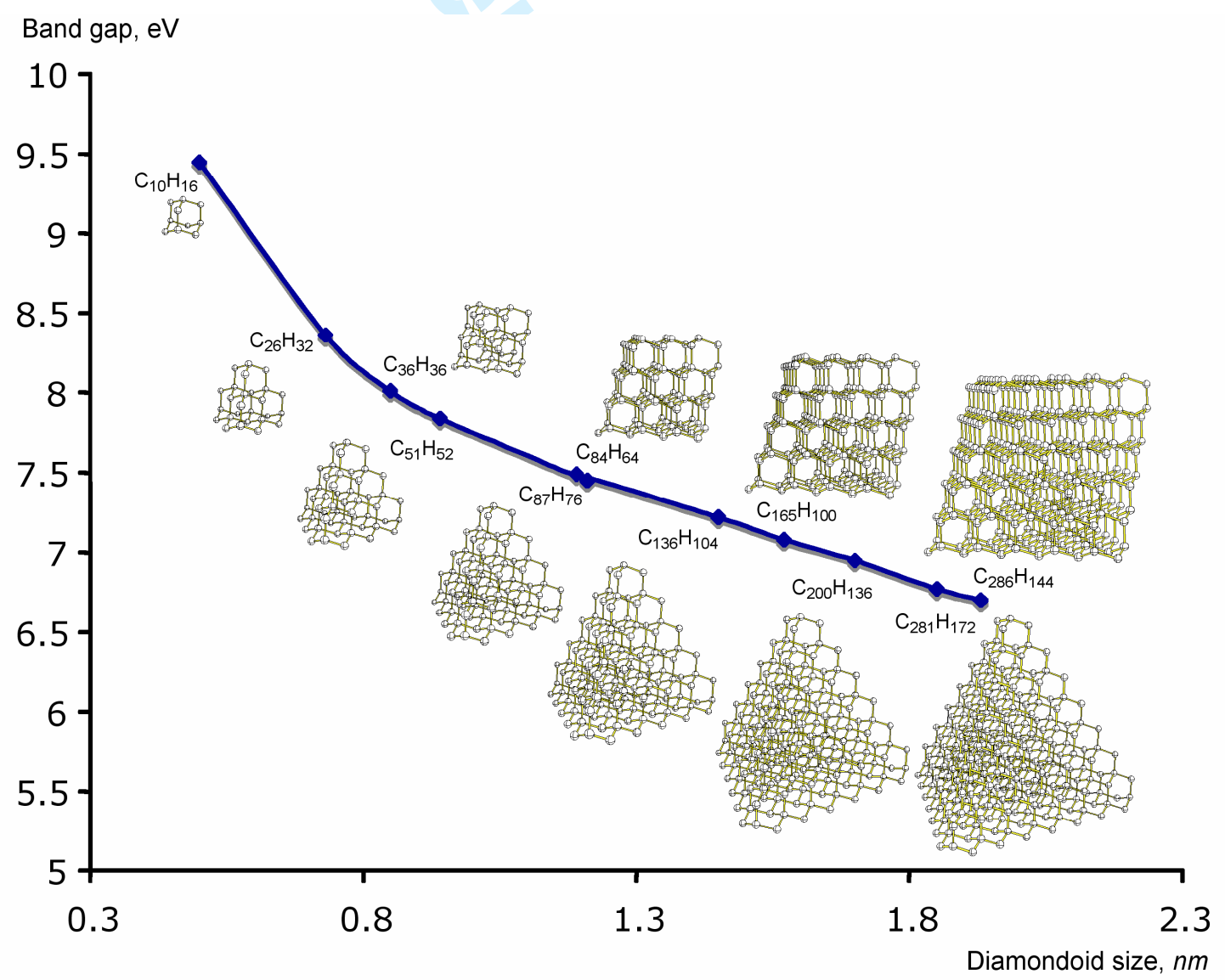

Figure 1. The dependence of the band gap of hydrogen-terminated nanodiamonds on particle size [B3LYP/6-31G(d), the surface hydrogens are omitted for clarity].

Earlier we predicted ${ }^{[11]}$ that the ionization potentials of diamondoids only slightly depend on the shape of the molecules but instead are mostly determined by the dimensions of the 
particles. Recently these B3LYP/6-31G(d) computations were confirmed experimentally by total-ion-yield spectroscopy of diamondoids. ${ }^{[35]}$ We now fully optimized at the B3LYP/631(d) level a number of tetrahedral hydrogen-terminated diamondoids from up to $\mathrm{C}_{281} \mathrm{H}_{172}$ and plotted these together with the data for the previously computed ${ }^{[34]}$ octahedral nanodiamonds (Figure 1). The results clearly demonstrate that the band gap rapidly decreases with increasing size, but even at ca. $2 \mathrm{~nm}$ it only reaches $6.7 \mathrm{eV}$; this is still much larger than that of bulk diamond $(5.5 \mathrm{eV})$. Even though the computed value for bulk diamond is not available at this level, strong quantum confinement effects still are expected for diamondoids above $2 \mathrm{~nm}$ in size. At the same time the trends in band gap changes are independent of the particle shapes - both tetrahedral and octahedral particles fit onto the same curve nicely (Figure 1).

Substitutional (external) doping. Many attempts to prepare functionalized nanodiamond led to inseparable inhomogeneous mixtures. ${ }^{[36]}$ These difficulties make precise tuning of the electronic properties of functionalized detonation or CVD nanodiamond rather difficult. As outlined above, functionalized diamondoids (nanodiamonds) represent chemically and physically homogeneous materials with well-defined electronic properties. For instance, our recent experimental studies have shown that diamondoid thiols ${ }^{[37]}$ form highly structured selfassembled monolayers on metal surfaces. ${ }^{[38]}$ We also found experimentally that [121]tetramantane-6-thiol (4) on gold and silver surfaces behaves as a high quantum yield monochromatic electron emitter. ${ }^{[37]}$ Thus, diamondoids offer unique opportunities to study the relationships between the substitution pattern and the properties of nanodiamond particles, ${ }^{[35,39]}$ and, in contrast to previous attempts with traditional bulk nanodiamond, allow the fine tuning of these properties of diamond at the nanolevel.

We computed the band gaps for a number of preparatively available mono- and disubstituted diamantanes as a model for "external" doping by substitution (Table 1). The difference between the B3PW91 and B3LYP results is negligible in the present case. Thus, the band gap of unsubstituted diamantane $\mathrm{C}_{14} \mathrm{H}_{20}$ is $9.0 \mathrm{eV}$ (entry 7) at B3PW91/6-31G(d,p), which is only slightly larger than the previously computed B3LYP/6-31G(d) value of 8.8 $\mathrm{eV}^{[11]}$ The B3PW91 adiabatic ionization potential $(8.4 \mathrm{eV})$ of diamantane is the same as that at B3LYP $(8.4 \mathrm{eV}) ;^{[40]}$ the vertical ionization potential computed at B3PW91 $(8.9 \mathrm{eV})$ is identical to the available experimental value. ${ }^{[41]}$

Substitution with alkyl, perfluoroalkyl groups as well as with fluorine increases the band gap in diamantane derivatives (entries 1-6) mostly due to stabilization of the occupied states 
(the HOMOs are downshifted by up to $0.03 \mathrm{au}$ ). While fluorination of nanodiamond was considered as very promising for several applications, ${ }^{[4]}$ we conclude, however, that the undesired insulating properties of nanodiamond materials will only increase after fluorination of the surface.

Remarkably, the band gap of bis-diadamantyl (entry 10) narrows only slightly relative to that of 2 ( $8.8 \mathrm{vs} 9.0 \mathrm{eV})$ despite a doubling of the dimensions of the molecule to $0.8 \mathrm{~nm}$; a band gap of ca $8 \mathrm{eV}$ may have been expected for a diamondoid of that size from Figure 1. We also computed the dimer of $\mathbf{4}$ and found that the band gap in this ca $1.8 \mathrm{~nm}$ structure is 8.4 $\mathrm{eV}$, close to that of the parent monomeric hydrocarbon $(8.6 \mathrm{eV})$. These observation allow us to predict that agglomeration of nanodiamond particles, despite a formal increase in size, will show only little quantum confinement effects. This may limit electronic applications of traditional nanodiamond, which consists of conglomerates, whose separation represents a formidable challenge. ${ }^{[42]}$

A substantial decrease of the band gap of $\mathbf{2}$ was achieved through incorporation of amino-, nitro-, and phenyl-functionalities. The substitution effects are not additive (compare, for instance, the mono- and dinitroderivatives, entries 19 and 21). The most electronwithdrawing group dominates the electronic properties of the substituted diamondoids. With nitro substitution the band gap of 2 can be reduced to ca. $6 \mathrm{eV}$ (entry 19-21). Outstanding semiconductor properties may be expected for the highly polarized betaine structure of the formal bis-apical amino acid derivative of $\mathbf{2}$ (entry 22).

Table 1. The computed HOMO/LUMO energies and energy separations for 4,9-disubstituted diamantanes (B3PW91/6-31G(d,p)).

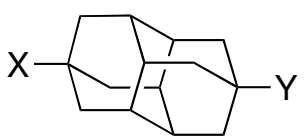

\begin{tabular}{ccccccc}
\hline$\#$ & $\mathrm{X}$ & $\mathrm{Y}$ & $\begin{array}{c}\text { HOMO, } \\
\mathrm{au}\end{array}$ & $\begin{array}{c}\text { LUMO, } \\
\mathrm{au}\end{array}$ & $\begin{array}{c}\text { HOMO-LUMO } \\
\text { gap, kcal/mol }\end{array}$ & $\begin{array}{c}\text { HOMO-LUMO } \\
\text { gap, eV }\end{array}$ \\
\hline 1 & $\mathrm{CF}_{3}$ & $\mathrm{CF}_{3}$ & -0.28682 & 0.06085 & 218.2 & 9.5 \\
2 & $\mathrm{CH}_{3}$ & $\mathrm{CF}_{3}$ & -0.27507 & 0.06753 & 215.0 & 9.3 \\
3 & $\mathrm{CH}_{3}$ & $\mathrm{CH}_{3}$ & -0.26302 & 0.07463 & 211.9 & 9.2 \\
4 & $\mathrm{CH}_{3}$ & $\mathrm{~F}$ & -0.27167 & 0.06354 & 210.3 & 9.1 \\
5 & $\mathrm{H}$ & $\mathrm{CH}_{3}$ & -0.26067 & 0.07362 & 209.8 & 9.1 \\
6 & $\mathrm{~F}$ & $\mathrm{~F}$ & -0.27928 & 0.05393 & 209.1 & 9.1 \\
\hline
\end{tabular}




\begin{tabular}{ccccccc}
7 & $\mathrm{H}$ & $\mathrm{H}$ & -0.25845 & 0.07273 & 207.8 & 9.0 \\
8 & $\mathrm{CN}$ & $\mathrm{CN}$ & -0.29816 & 0.03153 & 206.9 & 9.0 \\
9 & $\mathrm{CH}_{3}$ & $\mathrm{CN}$ & -0.28111 & 0.04421 & 204.1 & 8.8 \\
10 & $\mathrm{H}$ & -0.25030 & 0.07344 & 203.1 & 8.8 \\
& & & & & \\
11 & $\mathrm{OH}$ & $\mathrm{OH}$ & -0.25278 & 0.06555 & 199.7 & 8.7 \\
12 & $\mathrm{CH}_{3}$ & $\mathrm{NH}_{2}$ & -0.22692 & 0.07326 & 188.4 & 8.2 \\
13 & $\mathrm{NH}_{2}$ & $\mathrm{NH}_{2}$ & -0.22738 & 0.07220 & 187.9 & 8.1 \\
14 & $\mathrm{CH} \mathrm{CH}_{2}$ & $\mathrm{~F}$ & -0.25199 & 0.01364 & 166.7 & 7.2 \\
15 & $\mathrm{H}$ & $\mathrm{NH}_{3}^{+}$ & -0.39239 & -0.13090 & 164.1 & 7.1 \\
16 & $\mathrm{NH}_{2}$ & $\mathrm{COOH}_{1}$ & -0.23055 & 0.01143 & 151.8 & 6.6 \\
17 & $\mathrm{Ph}_{18}$ & $\mathrm{Ph}^{2}$ & -0.24506 & -0.00602 & 150.0 & 6.5 \\
19 & $\mathrm{NO}_{2}$ & $\mathrm{NO}_{2}$ & -0.24084 & -0.00212 & 149.8 & 6.5 \\
20 & $\mathrm{CH}_{3}$ & $\mathrm{NO}_{2}$ & -0.27893 & -0.06691 & 140.9 & 6.1 \\
21 & $\mathrm{H}$ & $\mathrm{NO}_{2}$ & -0.27789 & -0.05669 & 139.5 & 6.0 \\
22 & $\mathrm{NH}_{3}^{+}$ & $\mathrm{COO}^{-}$ & -0.11669 & -0.05125 & 41.1 & 1.8 \\
\hline
\end{tabular}

Interstitial (internal) doping. The semiconductor properties of natural bulk diamond are determined by trace amounts of nitrogen (type I) or boron (type II) impurities. Modeling these effects by placing the dopant atom in the center of spherical diamond nanocrystals largely leads to unstable structures. ${ }^{[43]}$ In contrast, substitutional internal doping in diamondoid does give rise to stable molecules. ${ }^{[33]}$ We first computed the band gap for the parent heteroadamantanes (Table 2) with oxygen, nitrogen as well as sulfur as n-, and boron as p-dopants. While the HOMO of n-doped structures (entries 2-4) mostly describe the nelectrons of the heteroatom, the empty $p$-orbital of boron interacts strongly with the diamondoid LUMOs. Remarkably, interstitial doping dramatically reduces the band gap down to about $7 \mathrm{eV}$ for the sulfur (entry 3 ) and boron (entry 6) derivatives.

Table 2. The computed HOMO/LUMO energies and gaps for doped adamantanes [B3PW91/6-31G(d,p)].

\begin{tabular}{ccccccc} 
HOMO, au & LUMO, au & $\begin{array}{c}\text { HOMO-LUMO gap, } \\
\text { kcal/mol }\end{array}$ & $\begin{array}{c}\text { HOMO-LUMO } \\
\text { gap, eV }\end{array}$ \\
\hline 1 & $\mathrm{CH}$ & $\mathrm{CH}_{2}$ & -0.27293 & 0.07434 & 217.9 & 9.4 \\
2 & $\mathrm{O}$ & $\mathrm{CH}_{2}$ & -0.23266 & 0.07550 & 193.4 & 8.4 \\
3 & $\mathrm{~S}$ & $\mathrm{CH}_{2}$ & -0.21058 & 0.04023 & 157.4 & 6.8
\end{tabular}




\begin{tabular}{lllllll}
\hline 4 & $\mathrm{CH}_{2}$ & $\mathrm{~N}$ & -0.19592 & 0.07653 & 171.0 & 7.4 \\
6 & $\mathrm{CH}_{2}$ & $\mathrm{~B}$ & -0.25846 & -0.00370 & 159.9 & 6.9 \\
\hline
\end{tabular}

Several questions arise: $i$ ) how does such internal doping influence the band gap for larger diamond particles, ii) are these effects additive and do they depend on the position of the dopant, and iii) to what extend do conducting and valence bands shrink upon enlarging the particles, i. e., what is the degree of mixing of the carbon and heteroatom orbitals. To answer these questions we fully optimized a number of diamantane (2) and triamantane (3) derivatives at B3PW91/6-31G(d,p). Internal doping of two different $\mathrm{CH}$ positions of the cage of 2 (entries 2 and 3) reduces the band gap almost equally relative to the undoped molecule (entry 1); the substitution of the $\mathrm{CH}_{2}$ moieties reduces the band gap to a lesser extent (entry 4). Importantly, double doping virtually provides no additional effect as compared to the incorporation of only one dopant (entry 5 vs 3); this will make the preparation of such molecules less challenging. In agreement with data for the adamantane derivatives, doping with oxygen (entries 6 and 7) is less effective, while sulfur (entries 8-10) and boron (entries 11 and 12) incorporation is more effective than $N$-doping. The most pronounced effect was found for push-pull doping with boron and nitrogen that reduces the band gap of 2 (entries 13 and 14) to that of bulk diamond.

Table 3. The computed HOMO/LUMO energies and gaps for doped diamantanes [B3PW91/6-31G(d,p)].

\begin{tabular}{|c|c|c|c|c|c|c|c|c|c|c|}
\hline$\#$ & $\mathrm{X}$ & $\mathrm{Y}$ & $\mathrm{Z}$ & $\mathrm{W}$ & $\mathrm{U}$ & $\mathrm{V}$ & $\begin{array}{c}\text { HOMO, } \\
\text { au }\end{array}$ & $\begin{array}{c}\text { LUMO, } \\
\text { au }\end{array}$ & $\begin{array}{c}\text { HOMO- } \\
\text { LUMO } \\
\text { gap, } \\
\text { kcal/mol }\end{array}$ & $\begin{array}{l}\text { HOMO- } \\
\text { LUMO } \\
\text { gap, eV }\end{array}$ \\
\hline 1 & $\mathrm{CH}_{2}$ & $\mathrm{CH}$ & $\mathrm{CH}$ & $\mathrm{CH}_{2}$ & $\mathrm{CH}_{2}$ & $\mathrm{CH}$ & -0.25845 & 0.07273 & 207.8 & 9.0 \\
\hline 2 & $\mathrm{CH}_{2}$ & $\mathrm{~N}$ & $\mathrm{CH}$ & $\mathrm{CH}_{2}$ & $\mathrm{CH}_{2}$ & $\mathrm{CH}$ & -0.19597 & 0.07607 & 170.7 & 7.4 \\
\hline 3 & $\mathrm{CH}_{2}$ & $\mathrm{CH}$ & $\mathrm{N}$ & $\mathrm{CH}_{2}$ & $\mathrm{CH}_{2}$ & $\mathrm{CH}$ & -0.19390 & 0.07402 & 168.1 & 7.3 \\
\hline 4 & $\mathrm{NH}$ & $\mathrm{CH}$ & $\mathrm{CH}$ & $\mathrm{CH}_{2}$ & $\mathrm{CH}_{2}$ & $\mathrm{CH}$ & -0.20722 & 0.07141 & 174.8 & 7.6 \\
\hline 5 & $\mathrm{CH}_{2}$ & $\mathrm{~N}$ & $\mathrm{CH}$ & $\mathrm{CH}_{2}$ & $\mathrm{~N}$ & $\mathrm{CH}$ & -0.18617 & 0.08200 & 168.3 & 7.3 \\
\hline
\end{tabular}




\begin{tabular}{ccccccccccc}
\hline 6 & $\mathrm{O}$ & $\mathrm{CH}$ & $\mathrm{CH}$ & $\mathrm{CH}_{2}$ & $\mathrm{CH}_{2}$ & $\mathrm{CH}$ & -0.23035 & 0.07181 & 189.6 & 8.2 \\
7 & $\mathrm{O}$ & $\mathrm{CH}$ & $\mathrm{CH}$ & $\mathrm{O}$ & $\mathrm{CH}_{2}$ & $\mathrm{CH}$ & -0.23064 & 0.07515 & 191.9 & 8.3 \\
8 & $\mathrm{~S}$ & $\mathrm{CH}$ & $\mathrm{CH}$ & $\mathrm{CH}_{2}$ & $\mathrm{CH}_{2}$ & $\mathrm{CH}$ & -0.21083 & 0.03945 & 157.0 & 6.8 \\
9 & $\mathrm{~S}$ & $\mathrm{CH}$ & $\mathrm{CH}$ & $\mathrm{S}$ & $\mathrm{CH}_{2}$ & $\mathrm{CH}$ & -0.20946 & 0.03453 & 153.1 & 6.6 \\
10 & $\mathrm{O}$ & $\mathrm{CH}$ & $\mathrm{CH}$ & $\mathrm{S}$ & $\mathrm{CH}_{2}$ & $\mathrm{CH}$ & -0.21510 & 0.03586 & 157.5 & 6.8 \\
11 & $\mathrm{CH}_{2}$ & $\mathrm{~B}$ & $\mathrm{CH}$ & $\mathrm{CH}_{2}$ & $\mathrm{CH}_{2}$ & $\mathrm{CH}$ & -0.25684 & -0.00512 & 157.9 & 6.8 \\
12 & $\mathrm{CH}_{2}$ & $\mathrm{~B}$ & $\mathrm{CH}$ & $\mathrm{CH}_{2}$ & $\mathrm{~B}$ & $\mathrm{CH}$ & -0.26093 & -0.00909 & 158.0 & 6.8 \\
13 & $\mathrm{CH}_{2}$ & $\mathrm{~N}$ & $\mathrm{CH}$ & $\mathrm{CH}_{2}$ & $\mathrm{~B}$ & $\mathrm{CH}$ & -0.20185 & -0.00854 & 121.3 & 5.3 \\
14 & $\mathrm{CH}_{2}$ & $\mathrm{CH}$ & $\mathrm{N}$ & $\mathrm{CH}_{2}$ & $\mathrm{CH}_{2}$ & $\mathrm{~B}$ & -0.20237 & -0.00605 & 123.2 & 5.3 \\
\hline
\end{tabular}

The trends found for our diamantane model are nicely reflected in the series of doped triamantanes (Table 4). The position of the dopant has a negligible influence of the HOMOLUMO gaps. Narrowing of the gap is determined only by the nature of the dopant, rather than by the size of the cage. This is due to the dominant contributions of the atomic orbitals of the dopant into the HOMOs and the LUMOs.

Table 4. The computed HOMO/LUMO energies and gaps for doped diamantanes [B3PW91/6-31G(d,p)].

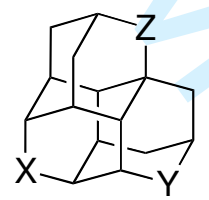

\begin{tabular}{ccccccc}
\hline $\mathrm{X}$ & $\mathrm{Y}$ & $\mathrm{Z}$ & $\mathrm{HOMO}, \mathrm{au}$ & $\mathrm{LUMO}, \mathrm{au}$ & $\begin{array}{c}\text { HOMO-LUMO gap, } \\
\mathrm{kcal} / \mathrm{mol}\end{array}$ & $\begin{array}{c}\text { HOMO-LUMO } \\
\mathrm{gap}, \mathrm{eV}\end{array}$ \\
\hline $\mathrm{CH}_{2}$ & $\mathrm{CH}_{2}$ & $\mathrm{CH}_{2}$ & -0.24929 & 0.07140 & 201.2 & 8.7 \\
$\mathrm{O}$ & $\mathrm{CH}_{2}$ & $\mathrm{CH}_{2}$ & -0.22779 & 0.07126 & 187.6 & 8.1 \\
$\mathrm{~S}$ & $\mathrm{CH}_{2}$ & $\mathrm{CH}_{2}$ & -0.20821 & 0.04330 & 157.8 & 6.8 \\
$\mathrm{CH}_{2}$ & $\mathrm{O}$ & $\mathrm{CH}_{2}$ & -0.22813 & 0.07111 & 187.8 & 8.1 \\
$\mathrm{CH}_{2}$ & $\mathrm{~S}$ & $\mathrm{CH}_{2}$ & -0.20885 & 0.04143 & 157.0 & 6.8 \\
$\mathrm{CH}_{2}$ & $\mathrm{CH}$ & $\mathrm{O}$ & -0.22821 & 0.07202 & 188.4 & 8.2 \\
$\mathrm{CH}_{2}$ & $\mathrm{CH}_{2}$ & $\mathrm{~S}$ & -0.20865 & 0.03907 & 155.4 & 6.7 \\
\hline
\end{tabular}

At the same time, increasing the size of the particle increases the densities of both the occupied and the unoccupied states, i. e., we observe the transition of discrete molecular 
orbital levels to band structures as the diamondoids become larger. The energies of the frontier and related molecular orbitals for six representative $S$-doped hydrocarbons are depicted in Fig. 2. While the densities of the unoccupied states increase only slightly the density of the valence bands increases considerably. That is, large doped nanodiamonds will behave as semiconductors, analogous to doped bulk diamond.

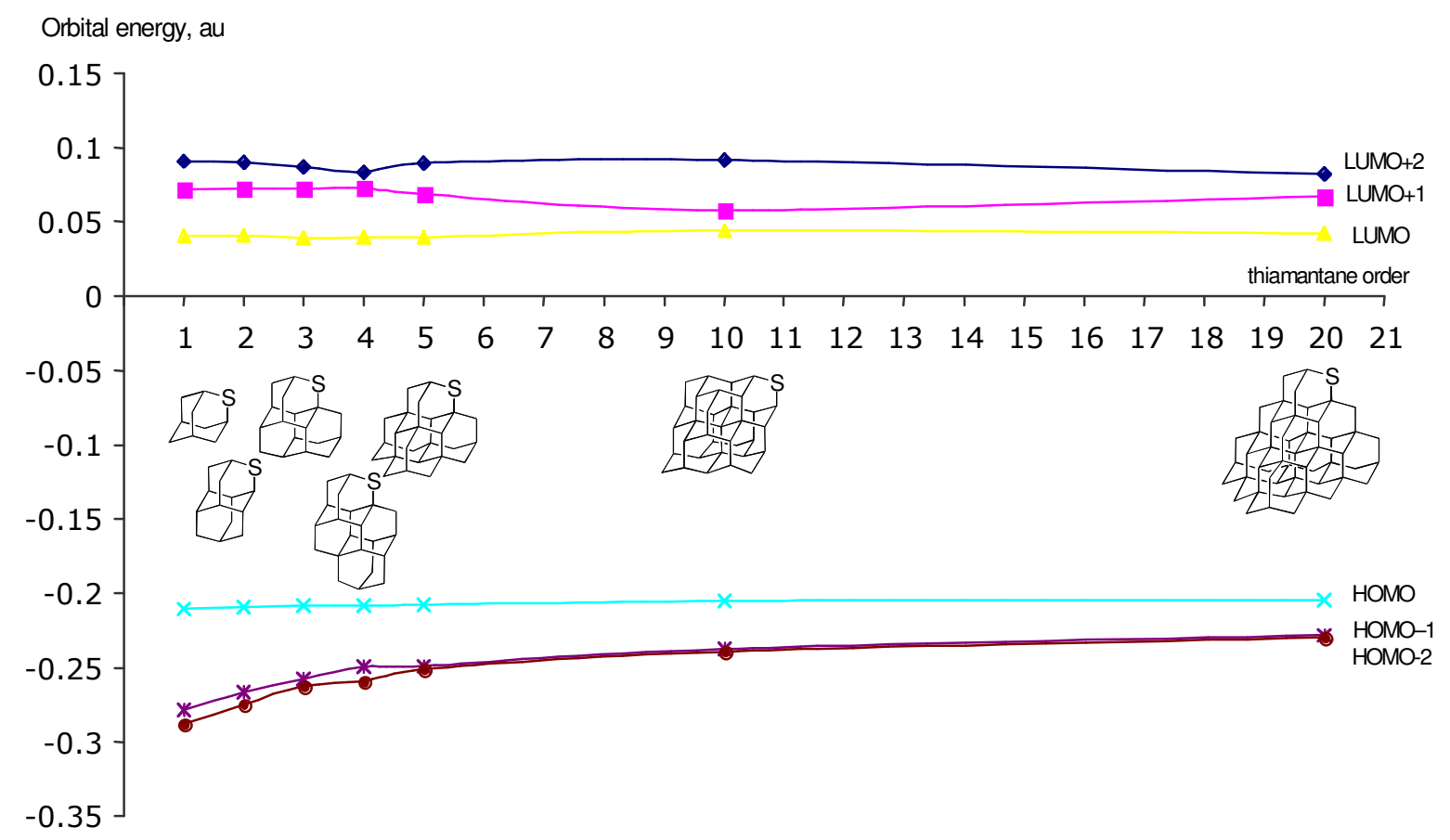

Figure 2. Changes in the densities of the unoccupied and occupied stated with increasing size of S-doped nanodiamonds [thiamantanes, at B3PW91/6-31G(d,p)].

\section{Conclusions}

Well-defined nanodiamonds display strong quantum confinement effects at particle sizes from 0.5 to at least $2 \mathrm{~nm}$, when their band gaps are reduced to $6.7 \mathrm{eV}$, which is likely to be still much wider than that of bulk diamond. Octahedral and tetrahedral nanodiamonds show the same trends in band gap narrowing, and it is the dimension rather than the shape/morphology of the nanodiamonds that affects the band gaps. Clustering of diamondoids apparently does not lead to substantial additive band-gap lowering as shown for the diamondoid dimers; this is also evident from most recent computations on diamondoid crystals. $^{[32]}$ 


\section{Acknowledgments}

This paper is dedicated to Fritz Schaefer for his many valuable contributions to computational as well as theoretical chemistry, and on the occasion of his $65^{\text {th }}$ birthday. We thank Trevor M. Willey, Jeremy E. P. Dahl, and Robert M. K. Carlson for fruitful discussions. This work was supported by the Deutsche Forschungsgemeinschaft, the Ministry of Science and Education of Ukraine, Ukrainian Basic Research Fund, and the Fonds der Chemischen Industrie. We thank the CSC Frankfurt for computational resources.

\section{References}

[1] J. E. Dahl, S. G. Liu, R. M. K. Carlson, Science 299, 96 (2003).

[2] a) A. P. Marchand, Science 299, 52 (2003); b) H. Hopf, Angew. Chem. Int. Ed. 42, 2000 (2003).

[3] A. S. Barnard, S. P. Russo, I. K. Snook, J. Chem. Phys. 118, 5094 (2003).

[4] M. Baidarakova, A. Vul', J. Phys. D: Appl. Phys. 40, 6300 (2007).

[5] W. P. Kang, J. L. Davidson, Y. M. Wong, K. Holmes, Diam. Relat. Mater. 13, 975 (2004).

[6] P. v. R. Schleyer, J. Am. Chem. Soc. 79, 3292 (1957).

[7] a) J. G. Henkel, J. T. Hane, J. Med. Chem. 25, 51 (1982); b) Y. N. Klimochkin, M. V. Leonova, I. R. Korzhev, I. K. Moiseyev, G. V. Vladyko, L. V. Korobchenko, Y. I. Boreko, S. N. Nikolayeva, Khim. Farm. Zh. 26, 58 (1992); c) W. J. Hoekstra, J. B. Press, M. P. Bonner, P. Angrade-Gordon, P. M. Keane, K. A. Durkin, D. C. Liotta, K. H. Mayo, Bioorg. Chem. Lett. 4, 1361 (1994); d) Y. I. Bagrii, Petroleum Chem. 35, 205 (1995); e) J. Kornhuber, G. Quack, W. Danysz, K. Jellinger, W. Danielczyk, W. Gsell, P. Riederer, Neuropharmacology 34, 713 (1995); f) Y. Kuroda, H. Ueda, H. Nozawa, H. Ogoshi, Tetrahedron Lett. 38, 7901 (1997); g) D. Gully, B. Labeeuw, R. Boigegrain, F. OuryDonat, A. Bachy, M. Poncelet, R. Steinberg, M. F. SuaudChagny, V. Santucci, N. Vita, F. Pecceu, C. LabbeJullie, P. Kitabgi, P. Soubrie, G. LeFur, J. P. Maffrand, J. Pharm. Exper. Therap. 280, 802 (1997); h) K. Kitagawa, N. Mizobuchi, T. Hama, T. Hibi, R. Konishi, S. Futaki, Chem. Pharm. Bull. 45, 1782 (1997); i) D. Ranganathan, V. Haridas, K. P. Madhusudanan, R. Roy, R. Nagaraj, G. B. John, J. Am. Chem. Soc. 119, 11578 (1997); j) S. Samnick, S. Ametamey, M. R. Gold, P. A. Schubiger, J. Label. Comp. Radiopharm. 39, 241 (1997); k) E. A. Blokhina, I. A. Sukhotina, A. Y. Bespalov, Eur. J. Pharm. 406, 227 (2000); 1) J. Kotlinska, G. Biala, Pol. J. Pharm. 52, 179 (2000); m) M. A. Rogawski, Amino Acids 19, 133 (2000); n) 
F. G. Costa, R. Frussa, L. F. Felicio, Eur. J. Pharm. 428, 97 (2001); o) J. M. Kelly, G. Quack, M. M. Miles, Antimicr. Agents Chemother. 45, 1360 (2001); p) I. S. Morozov, I. A. Ivanova, T. A. Lukicheva, Khim. Farm. Zhurn. 35, 235 (2001); r) A. Baxter, J. Bent, K. Bowers, M. Braddock, S. Brough, M. Fagura, M. Lawson, T. McInally, M. Mortimore, M. Robertson, R. Weaver, W. P., Bioorg. Med. Chem. Lett. 13, 4047 (2003).

[8] T. Courtney, D. E. Johnston, M. A. McKervey, J. J. Rooney, J. Chem. Soc., Perkin Trans. 12691 (1972).

[9] a) V. Z. Williams, P. v. R.Schleyer, G. J. Gleicher, L. B. Rodewald, J. Am. Chem. Soc. 88, 3862 (1966); b) F. S. Hollowood, M. A. McKervey, R. Hamilton, J. J. Rooney, J. Org. Chem. 45, 4954 (1980).

[10] a) W. Burns, M. A. McKervey, T. R. B. Mitchell, J. J. Rooney, J. Am. Chem. Soc. 100, 906 (1978); b) W. Burns, T. R. B. Mitchell, M. A. McKervey, J. J. Rooney, G. Ferguson, P. Roberts, J. Chem. Soc. Chem. Comm. 893 (1976).

[11] A. A. Fokin, B. A. Tkachenko, P. A. Gunchenko, D. V. Gusev, P. R. Schreiner, Chem. Eur. J. 11, 7091 (2005).

[12] a) P. R. Schreiner, N. A. Fokina, B. A. Tkachenko, H. Hausmann, M. Serafin, J. E. P. Dahl, S. G. Liu, R. M. K. Carlson, A. A. Fokin, J. Org. Chem. 71, 6709 (2006); b) B. A. Tkachenko, N. A. Fokina, L. V. Chernish, J. E. P. Dahl, S. G. Liu, R. M. K. Carlson, A. A. Fokin, P. R. Schreiner, Org. Lett. 8, 1767 (2006); c) A. A. Fokin, E. D. Butova, L. V. Chernish, N. A. Fokina, J. E. P. Dahl, R. M. K. Carlson, P. R. Schreiner, Org. Lett. 9, 2541 (2007); d) N. A. Fokina, B. A. Tkachenko, A. Merz, M. Serafin, J. E. P. Dahl, R. M. K. Carlson, A. A. Fokin, P. R. Schreiner, Eur. J. Org. Chem. 4738 (2007).

[13] H. Schwertfeger, A. A. Fokin, P. R. Schreiner, Angew. Chem. Int. Ed. 47, 1022 (2008).

[14] M. Schoell, R. M. K. Carlson, Nature 399, 15 (1999).

[15] R. Lin, Z. A. Wilk, Fuel 74, 1512 (1995).

[16] J. E. Dahl, J. M. Moldowan, K. E. Peters, G. E. Claypool, M. A. Rooney, G. E. Michael, M. R. Mello, M. L. Kohnen, Nature 399, 54 (1999).

[17] A. A. Fokin, P. R. Schreiner, N. A. Fokina, B. A. Tkachenko, H. Hausmann, M. Serafin, J. E. P. Dahl, S. G. Liu, R. M. K. Carlson, J. Org. Chem. 71, 8532 (2006).

[18] a) A. S. Barnard, S. P. Russo, I. K. Snook, J. Phys. Chem. B 109, 11991 (2005); b) A. V. G. Chizmeshya, C. Ritter, J. Tolle, C. Cook, J. Menendez, J. Kouvetakis, Chem. Mater. 18, 6266 (2006); c) H. Uetsuka, D. Shin, N. Tokuda, K. Saeki, C. E. Nebel, Langmuir 23, 3466 (2007).

[19] M. Shen, H. F. Schaefer, C. Liang, J. H. Lii, N. L. Allinger, P. v. R. Schleyer, J. Am. Chem. Soc. 114, 497 (1992).

[20] T. M. Willey, C. Bostedt, T. van Buuren, J. E. Dahl, S. G. Liu, R. M. K. Carlson, L. J. Terminello, T. Möller, Phys. Rev. Lett. 95, 113401 (2005).

[21] T. M. Willey, C. Bostedt, T. van Buuren, J. E. Dahl, S. G. Liu, R. M. K. Carlson, R. W. Meulenberg, E. J. Nelson, L. J. Terminello, Phys. Rev. 74, 205432 (2006).

[22] M. J. Frisch, et., al., Gaussian, Inc., Pittsburgh PA, 2004.

[23] a) A. D. Becke, Phys. Rev. A 38, 3098 (1988); b) C. Lee, W. Yang, R. G. Parr, Phys. Rev. B 37, 785 (1988).

[24] J. P. Perdew, Y. Wang, Phys. Rev. B 23, 5048 (1992).

[25] a) P. R. Schreiner, A. A. Fokin, R. A. Pascal, A. de Meijere, Org. Lett. 8, 3635 (2006);

b) P. R. Schreiner, Angew. Chem. Int. Ed. 23, 4217 (2007).

[26] P. C. Hariharan, J. A. Pople, Theoret. Chim. Acta 28, 213 (1973). 
[27] a) J. Y. Raty, G. Galli, C. Bostedt, T. W. van Buuren, L. J. Terminello, Phys. Rev. Lett. 90, 0374011 (2003); b) B. Wen, J. Zhao, T. Li, Chem. Phys. Lett. 441, 318 (2007).

[28] N. D. Drummond, A. J. Williamson, R. J. Needs, G. Galli, Phys. Rev. Lett. 95, 096801 (2005).

[29] D. A. Areshkin, O. A. Shenderova, S. P. Adiga, D. W. Brenner, Diam. Relat. Mater. 13, 1826 (2004).

[30] T. M. Willey, C. Bostedt, T. van Buuren, J. E. Dahl, S. G. Liu, R. M. K. Carlson, R. W. Meulenberg, E. J. Nelson, L. J. Terminello, Phys. Rev. 74, 205432 (2006).

[31] Y. Wang, E. Kioupakis, X. Liu, D. Wegner, R. Yamachika, J. E. Dahl, R. M. K. Carlson, S. G. Louie, M. F. Crommie, Nat. Mater. 7, 38 (2008).

[32] T. Sasagawa, Z.-x. Shen, J. Appl. Phys. 104, 073704 (2008).

[33] G. C. McIntosh, M. Yoon, S. Berber, D. Tománek, Phys. Rev. B 70, 045401 (2004).

[34] M. Linnolahti, A. J. Karttunen, T. A. Pakkanen, J. Phys. Chem. 111, 18118 (2007).

[35] K. Lenzke, L. Landt, M. Hoener, H. Thomas, J. E. Dahl, S. G. Liu, R. M. K. Carlson, T. Möller, C. Bostedt, J. Chem. Phys. 127, 084320 (2007).

[36] a) A. Kruger, Angew. Chem. Int. Ed. 45, 6426 (2006); b) A. Kruger, Y. Liang, G. Jarre, J. Stegk, J. Mater. Chem. 16, 2322 (2006); c) L. Li, J. L. Davidson, C. M. Lukehart, Carbon 44, 2308 (2006); d) V. N. Khabasheskua, J. L. F. Margravea, E. V. Barrera, Diam. Relat. Mater. 14, 859 (2005); e) Y. Liu, Z. Gu, J. L. Margrave, V. N. Khabashesku, Chem. Mater. 16, 3924 (2004).

[37] W. L. Yang, J. D. Fabbri, T. M. Willey, J. R. I. Lee, J. E. Dahl, R. M. K. Carlson, P. R. Schreiner, A. A. Fokin, B. A. Tkachenko, N. A. Fokina, W. Meevasana, N. Mannella, K. Tanaka, X. J. Zhou, T. van Buuren, M. A. Kelly, Z. Hussain, N. A. Melosh, Z.-x. Shen, Science 316, 1460 (2007).

[38] T. M. Willey, J. D. Fabbri, J. R. I. Lee, P. R. Schreiner, A. A. Fokin, B. A. Tkachenko, N. A. Fokina, J. E. P. Dahl, R. M. K. Carlson, A. L. Vance, W. Yang, L. J. Terminello, T. v. Buuren, N. A. Melosh, J. Am. Chem. Soc. 130, 10536 (2008).

[39] P.-H. Chung, E. Perevedentseva, C.-L. Cheng, Surf. Sci. 601, 3866 (2007).

[40] a) A. J. Lu, B. C. Pan, J. G. Han, Phys. Rev. B. 72, 035447 (erratum: 209901) (2005); b) A. A. Fokin, B. A. Tkachenko, P. A. Gunchenko, D. V. Gusev, P. R. Schreiner, Chem. Eur. J. 11, 7091 (2005).

[41] M. J. S. Dewar, S. D. Worley, J. Chem. Phys. 50, 654 (1969).

[42] A. Kruger, F. Kataoka, M. Ozawa, T. Fujino, Y. Suzuki, A. E. Aleksenskii, A. Y. Vul', E. Osawa, Carbon 43, 1722 (2005).

[43] a) A. S. Barnard, S. P. Russo, I. K. Snook, J. Phys. Chem. B. 109, 11991 (2005); b) A. S. Barnard, S. P. Russo, I. K. Snook, J. Chem. Phys. 118, 10725 (2003). 


\title{
SUPPLEMENTARY INFORMATION
}

\section{Band Gap Tuning in Nanodiamonds: First Principle Computational Studies}

\author{
Andrey A. Fokin ${ }^{\mathrm{a}, \mathrm{b}^{*}}$ and Peter R. Schreiner ${ }^{\mathrm{b} *}$ \\ ${ }^{a}$ Department of Organic Chemistry, Kiev Polytechnic Institute, pr. Pobedy 37, 03056 Kiev, \\ Ukraine and ${ }^{b}$ Institute of Organic Chemistry, Justus-Liebig University, Heinrich-Buff-Ring 58, D- \\ 35392 Giessen, Germany
}

Table 1s. The XYZ-coordinates of optimized molecular geometries and absolute energies in au.

$\begin{array}{lrrr}\mathrm{C}_{10} \mathrm{H}_{16}, \mathrm{~B} 3 L Y P / 6-31 \mathrm{G}^{*}, \mathrm{E}=-390.72601 \\ \mathrm{C} & 0.892683 & 0.892683 & 0.892683 \\ \mathrm{C} & -0.892683 & -0.892683 & 0.892683 \\ \mathrm{C} & -0.892683 & 0.892683 & -0.892683 \\ \mathrm{C} & 0.892683 & -0.892683 & -0.892683 \\ \mathrm{C} & 0.000000 & 0.000000 & 1.781020 \\ \mathrm{C} & 0.000000 & 1.781020 & 0.000000 \\ \mathrm{C} & 1.781020 & 0.000000 & 0.000000 \\ \mathrm{C} & -1.781020 & 0.000000 & 0.000000 \\ \mathrm{C} & 0.000000 & -1.781020 & 0.000000 \\ \mathrm{C} & 0.000000 & 0.000000 & -1.781020 \\ \mathrm{H} & -0.623944 & 0.623944 & 2.436301 \\ \mathrm{H} & 0.623944 & -0.623944 & 2.436301 \\ \mathrm{H} & 0.623944 & 2.436301 & -0.623944 \\ \mathrm{H} & -0.623944 & 2.436301 & 0.623944 \\ \mathrm{H} & 2.436301 & -0.623944 & 0.623944 \\ \mathrm{H} & 2.436301 & 0.623944 & -0.623944 \\ \mathrm{H} & -2.436301 & -0.623944 & -0.623944 \\ \mathrm{H} & -2.436301 & 0.623944 & 0.623944 \\ \mathrm{H} & -0.623944 & -0.623944 & -2.436301 \\ \mathrm{H} & 0.623944 & 0.623944 & -2.436301 \\ \mathrm{H} & -0.623944 & -2.436301 & -0.623944 \\ \mathrm{H} & 0.623944 & -2.436301 & 0.623944 \\ \mathrm{H} & 1.526783 & 1.526783 & 1.526783 \\ \mathrm{H} & -1.526783 & -1.526783 & 1.526783 \\ \mathrm{H} & -1.526783 & 1.526783 & -1.526783 \\ \mathrm{H} & 1.526783 & -1.526783 & -1.526783\end{array}$

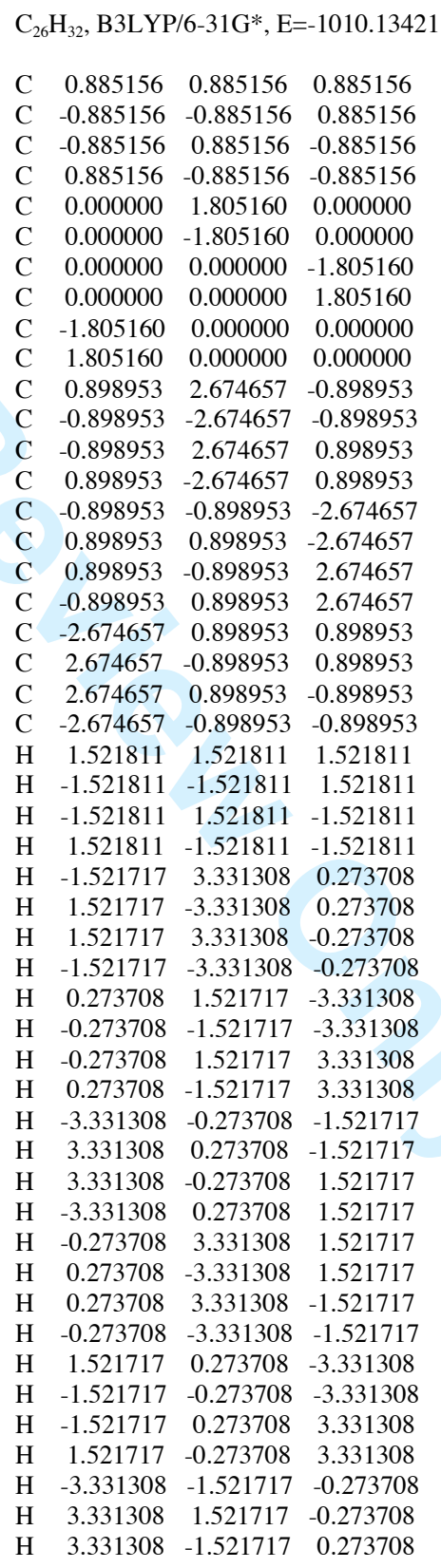

*Corresponding authors: aaf@xtf.ntu-kpi.kiev.ua and prs@org.chemie.uni-giessen.de

URL: http://mc.manuscriptcentral.com/tandf/tmph 


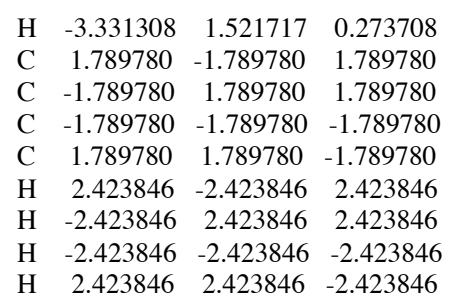

$$
\begin{aligned}
& \mathrm{C}_{51} \mathrm{H}_{52}, \mathrm{~B} 3 \mathrm{LYP} / 6-31 \mathrm{G}^{*}, \mathrm{E}=--1974.9449 \\
& \begin{array}{llll}
\text { C } & -0.898555 & -0.898555 & 0.898555
\end{array} \\
& \begin{array}{llll}
\text { C } & 0.898555 & 0.898555 & 0.898555
\end{array} \\
& \begin{array}{llll}
\text { C } & 0.898555 & -0.898555 & -0.898555
\end{array} \\
& \begin{array}{llll}
\text { C } & -0.898555 & 0.898555 & -0.898555
\end{array} \\
& \begin{array}{llll}
\text { C } & -0.898297 & 0.898297 & 2.700887
\end{array} \\
& \begin{array}{llll}
\text { C } & 0.898297 & -0.898297 & 2.700887
\end{array} \\
& \begin{array}{llll}
\text { C } & 0.898297 & 0.898297 & -2.700887
\end{array} \\
& \begin{array}{llll}
\text { C } & -0.898297 & -0.898297 & -2.700887
\end{array} \\
& \begin{array}{llll}
\text { C } & -0.898297 & 2.700887 & 0.898297
\end{array} \\
& \begin{array}{llll}
\text { C } & 0.898297 & -2.700887 & 0.898297
\end{array} \\
& \begin{array}{llll}
\text { C } & 0.898297 & 2.700887 & -0.898297
\end{array} \\
& \begin{array}{llll}
\text { C } & -0.898297 & -2.700887 & -0.898297
\end{array} \\
& \begin{array}{llll}
\text { C } & -2.700887 & 0.898297 & 0.898297
\end{array} \\
& \begin{array}{llll}
\text { C } & 2.700887 & -0.898297 & 0.898297
\end{array} \\
& \begin{array}{llll}
\text { C } & 2.700887 & 0.898297 & -0.898297
\end{array} \\
& \begin{array}{llll}
\text { C } & -2.700887 & -0.898297 & -0.898297
\end{array} \\
& \begin{array}{llll}
\text { C } & -1.782104 & 0.014063 & 1.782104
\end{array} \\
& \begin{array}{llll}
\text { C } & 1.782104 & -0.014063 & 1.782104
\end{array} \\
& \begin{array}{llll}
\text { C } & 1.782104 & 0.014063 & -1.782104
\end{array} \\
& \begin{array}{llll}
\text { C } & -1.782104 & -0.014063 & -1.782104
\end{array} \\
& \begin{array}{llll}
\text { C } & -0.014063 & 1.782104 & 1.782104
\end{array} \\
& \begin{array}{llll}
\text { C } & 0.014063 & -1.782104 & 1.782104
\end{array} \\
& \begin{array}{llll}
\text { C } & 0.014063 & 1.782104 & -1.782104
\end{array} \\
& \begin{array}{llll}
\text { C } & -0.014063 & -1.782104 & -1.782104
\end{array} \\
& \begin{array}{llll}
\text { C } & -1.782104 & 1.782104 & 0.014063
\end{array} \\
& \begin{array}{llll}
\text { C } & 1.782104 & -1.782104 & 0.014063
\end{array} \\
& \begin{array}{llll}
\text { C } & 1.782104 & 1.782104 & -0.014063
\end{array} \\
& \begin{array}{llll}
\text { C } & -1.782104 & -1.782104 & -0.014063
\end{array} \\
& \begin{array}{llll}
\text { C } & 0.000000 & 0.000000 & 0.000000
\end{array} \\
& \begin{array}{llll}
\text { C } & 0.000000 & 3.566813 & 0.000000
\end{array} \\
& \begin{array}{llll}
\text { C } & 0.000000 & -3.566813 & 0.000000
\end{array} \\
& \begin{array}{llll}
\text { C } & -3.566813 & 0.000000 & 0.000000
\end{array} \\
& \begin{array}{llll}
\text { C } & 3.566813 & 0.000000 & 0.000000
\end{array} \\
& \begin{array}{llll}
\text { C } & 0.000000 & 0.000000 & 3.566813
\end{array} \\
& \begin{array}{llll}
\text { C } & 0.000000 & 0.000000 & -3.566813
\end{array} \\
& \mathrm{H} \quad 2.417040 \quad 2.417040 \quad 0.625854 \\
& \mathrm{H} \quad-2.417040 \quad-2.417040 \quad 0.625854 \\
& \mathrm{H} \quad-2.417040 \quad 2.417040 \quad-0.625854 \\
& \text { H } \quad 2.417040 \quad-2.417040 \quad-0.625854 \\
& \mathrm{H} \quad-2.417040 \quad 0.625854 \quad-2.417040 \\
& \text { H } \quad 2.417040 \quad-0.625854 \quad-2.417040 \\
& \begin{array}{llll}
\mathrm{H} & 2.417040 & 0.625854 & 2.417040
\end{array} \\
& \mathrm{H} \quad-2.417040 \quad-0.625854 \quad 2.417040 \\
& \text { H } \quad-0.625854 \quad 2.417040 \quad-2.417040 \\
& \text { H } \quad 0.625854 \quad-2.417040 \quad-2.417040 \\
& \text { H } \quad 0.625854 \quad 2.417040 \quad 2.417040 \\
& \mathrm{H} \quad-0.625854 \quad-2.417040 \quad 2.417040 \\
& \begin{array}{llll}
\text { C } & -3.572596 & 1.796460 & 1.796460
\end{array} \\
& \begin{array}{llll}
\text { C } & 3.572596 & -1.796460 & 1.796460
\end{array} \\
& \begin{array}{llll}
\text { C } & 3.572596 & 1.796460 & -1.796460
\end{array} \\
& \text { C } \quad-3.572596 \quad-1.796460 \quad-1.796460 \\
& \begin{array}{llll}
\text { C } & -1.796460 & 3.572596 & 1.796460
\end{array} \\
& \begin{array}{llll}
\text { C } & 1.796460 & -3.572596 & 1.796460
\end{array} \\
& \begin{array}{llll}
\text { C } & 1.796460 & 3.572596 & -1.796460
\end{array} \\
& \text { C } \quad-1.796460 \quad-3.572596 \quad-1.796460 \\
& \begin{array}{llll}
\text { C } & -1.796460 & 1.796460 & 3.572596
\end{array} \\
& \begin{array}{llll}
\text { C } & 1.796460 & -1.796460 & 3.572596
\end{array} \\
& \text { C } \quad 1.796460 \quad 1.796460 \quad-3.572596
\end{aligned}
$$$$
\text { C } \quad-1.796460 \quad-1.796460 \quad-3.572596
$$

$\mathrm{C}_{87} \mathrm{H}_{76}, \mathrm{~B} 3 \mathrm{LYP} / 6-31 \mathrm{G}^{*}, \mathrm{E}=-3361.41152$

$\begin{array}{llll}\text { C } & 0.000000 & 0.000000 & 0.000000\end{array}$

$\begin{array}{llll}\text { C } & 1.795873 & 1.795873 & 0.000973\end{array}$

$\begin{array}{llll}\text { C } & -1.795873 & -1.795873 & 0.000973\end{array}$

$\begin{array}{llll}\text { C } & -1.795873 & 1.795873 & -0.000973\end{array}$

$\begin{array}{llll}\text { C } & 1.795873 & -1.795873 & -0.000973\end{array}$

$\begin{array}{llll}\text { C } & 1.795873 & -0.000973 & -1.795873\end{array}$

$\begin{array}{llll}\text { C } & -1.795873 & 0.000973 & -1.795873\end{array}$

$\begin{array}{llll}\text { C } & -1.795873 & -0.000973 & 1.795873\end{array}$

$\begin{array}{llll}\text { C } & 1.795873 & 0.000973 & 1.795873\end{array}$

$\begin{array}{llll}\text { C } & -0.000973 & 1.795873 & -1.795873\end{array}$

$\begin{array}{llll}\text { C } & 0.000973 & -1.795873 & -1.795873\end{array}$

$\begin{array}{llll}\text { C } & 0.000973 & 1.795873 & 1.795873\end{array}$

$\begin{array}{llll}\text { C } & -0.000973 & -1.795873 & 1.795873\end{array}$

$\begin{array}{llll}\text { C } & 1.795528 & 1.795528 & -3.598365\end{array}$

$\begin{array}{llll}\text { C } & -1.795528 & -1.795528 & -3.598365\end{array}$

$\begin{array}{llll}\text { C } & -1.795528 & 1.795528 & 3.598365\end{array}$

$\begin{array}{llll}\text { C } & 1.795528 & -1.795528 & 3.598365\end{array}$

$\begin{array}{llll}\text { C } & 0.000000 & 0.000000 & -3.595288\end{array}$

$\begin{array}{llll}\text { C } & 0.000000 & 0.000000 & 3.595288\end{array}$

$\begin{array}{llll}\text { C } & 0.000000 & 3.595288 & 0.000000\end{array}$

$\begin{array}{llll}\text { C } & 0.000000 & -3.595288 & 0.000000\end{array}$

$\begin{array}{llll}\text { C } & -1.795527 & -3.598365 & -1.795528\end{array}$

$\begin{array}{llll}\text { C } & -1.795528 & 3.598365 & 1.795528\end{array}$

$\begin{array}{llll}\text { C } & 1.795528 & -3.598365 & 1.795528\end{array}$

$\begin{array}{llll}\text { C } & 3.595288 & 0.000000 & 0.000000\end{array}$

$\begin{array}{llll}\text { C } & -3.595288 & 0.000000 & 0.000000\end{array}$

$\begin{array}{llll}\text { C } & 3.598365 & 1.795528 & -1.795528\end{array}$

$\begin{array}{llll}\text { C } & -3.598365 & -1.795528 & -1.795528\end{array}$

$\begin{array}{llll}\text { C } & -3.598365 & 1.795528 & 1.795528\end{array}$

$\begin{array}{llll}\text { C } & 3.598365 & -1.795527 & 1.795527\end{array}$

$\begin{array}{llll}\text { C } & -0.896703 & 0.896703 & 0.896703\end{array}$

$\begin{array}{llll}\text { C } & 0.896703 & -0.896703 & 0.896703\end{array}$

$\begin{array}{lllll}\text { C } & 0.896703 & 0.896703 & -0.896703\end{array}$

$\begin{array}{llll}\text { C } & -0.896703 & -0.896703 & -0.896703\end{array}$

$\begin{array}{lllll}\text { C } & 2.676780 & 0.883388 & 0.883388\end{array}$

$\begin{array}{llll}\text { C } & -2.676780 & 0.883388 & -0.883388\end{array}$

$\begin{array}{llll}\text { C } & 2.676780 & -0.883388 & -0.883388\end{array}$

$\begin{array}{lllll}\text { C } & 2.678989 & 0.911107 & -2.678989\end{array}$

$\begin{array}{llll}\text { C } & -2.678989 & -0.911107 & -2.678989\end{array}$

$\begin{array}{llll}\text { C } & -2.678989 & 0.911107 & 2.678989\end{array}$

$\begin{array}{llll}\text { C } & 2.678989 & -0.911107 & 2.678989\end{array}$

$\begin{array}{llll}\text { C } & -0.883388 & 0.883388 & -2.676780\end{array}$

$\begin{array}{llll}\text { C } & 0.883388 & -0.883388 & -2.676780\end{array}$

$\begin{array}{llll}\text { C } & 0.883388 & 0.883388 & 2.676780\end{array}$

$\begin{array}{llll}\text { C } & -0.883388 & -0.883388 & 2.676780\end{array}$

$\begin{array}{llll}\text { C } & 0.883388 & 2.676780 & 0.883388\end{array}$

$\begin{array}{llll}\text { C } & -0.883388 & -2.676780 & 0.883388\end{array}$

$\begin{array}{lllll}\text { C } & -0.883388 & 2.676780 & -0.883388\end{array}$

$\begin{array}{llll}\text { C } & 0.883388 & -2.676780 & -0.883388\end{array}$

$\begin{array}{llll}\text { C } & 0.911107 & 2.678989 & -2.678989\end{array}$

$\begin{array}{llll}\text { C } & -0.911107 & -2.678989 & -2.678989\end{array}$

$\begin{array}{llll}\text { C } & -0.911107 & 2.678989 & 2.678989\end{array}$

$\begin{array}{llll}\text { C } & 0.911107 & -2.678989 & 2.678989\end{array}$

$\begin{array}{llll}\text { C } & 2.678989 & 2.678989 & -0.911107\end{array}$

$\begin{array}{llll}\text { C } & -2.678989 & -2.678989 & -0.911107\end{array}$

$\begin{array}{llll}\text { C } & -2.678989 & 2.678989 & 0.911107\end{array}$

$\begin{array}{llll}\text { C } & 2.678989 & -2.678989 & 0.911107\end{array}$ $\begin{array}{llll}\text { C } & 1.795528 & 3.598365 & -1.795528\end{array}$

$\begin{array}{llll}\text { C } & -2.676780 & -0.883388 & 0.883388\end{array}$ 
$\begin{array}{llll}\mathrm{H} & -0.624047 & 4.224135 & -0.624047\end{array}$

$\begin{array}{llll}\mathrm{H} & 0.624047 & -4.224135 & -0.624047\end{array}$

$\begin{array}{llll}\mathrm{H} & 0.624047 & 4.224135 & 0.624047\end{array}$

$\mathrm{H} \quad-0.624047 \quad-4.224135 \quad 0.624047$

$\begin{array}{llll}\mathrm{H} & -4.224135 & 0.624047 & -0.624047\end{array}$

$\begin{array}{llll}\mathrm{H} & 4.224135 & -0.624047 & -0.624047\end{array}$

$\begin{array}{llll}\mathrm{H} & 4.224135 & 0.624047 & 0.624047\end{array}$

$\begin{array}{llll}\mathrm{H} & -4.224135 & -0.624047 & 0.624047\end{array}$

$\begin{array}{llll}\mathrm{H} & 0.624047 & 0.624047 & 4.224135\end{array}$

$\mathrm{H} \quad-0.624047 \quad-0.624047 \quad 4.224135$

$\begin{array}{llll}\mathrm{H} & -0.624047 & 0.624047 & -4.224135\end{array}$

H $\quad 0.624047 \quad-0.624047 \quad-4.224135$

$\begin{array}{llll}\mathrm{H} & -1.170512 & 4.228595 & 2.418574\end{array}$

$\begin{array}{llll}\mathrm{H} & 1.170512 & -4.228595 & 2.418574\end{array}$

H $1.170512 \quad 4.228595 \quad-2.418574$

$\mathrm{H} \quad-1.170512 \quad-4.228595 \quad-2.418574$

$\begin{array}{llll}\mathrm{H} & -2.418574 & 4.228595 & 1.170512\end{array}$

H $\quad 2.418574 \quad-4.228595 \quad 1.170512$

$\mathrm{H} \quad 2.418574 \quad 4.228595 \quad-1.170512$

$\mathrm{H} \quad-2.418574 \quad-4.228595 \quad-1.170512$

$\begin{array}{llll}\mathrm{H} & -4.228595 & 1.170512 & 2.418574\end{array}$

$\begin{array}{llll}\mathrm{H} & 4.228595 & -1.170512 & 2.418574\end{array}$

H $\quad 4.228595 \quad 1.170512 \quad-2.418574$

H $\quad-4.228595 \quad-1.170512 \quad-2.418574$

$\begin{array}{llll}\mathrm{H} & -4.228595 & 2.418574 & 1.170512\end{array}$

H $\quad 4.228595 \quad-2.418574 \quad 1.170512$

$\mathrm{H} \quad 4.228595 \quad 2.418574 \quad-1.170512$

$\mathrm{H} \quad-4.228595 \quad-2.418574 \quad-1.170512$

$\mathrm{H} \quad-1.170512 \quad 2.418574 \quad 4.228595$

H $\quad 1.170512 \quad-2.418574 \quad 4.228595$

H $\quad 1.170512 \quad 2.418574 \quad-4.228595$

H $\quad-1.170512 \quad-2.418574 \quad-4.228595$

$\begin{array}{llll}\text { C } & -2.687543 & 2.687543 & 2.687543\end{array}$

$\begin{array}{llll}\text { C } & 2.687543 & -2.687543 & 2.687543\end{array}$

$\begin{array}{llll}\text { C } & 2.687543 & 2.687543 & -2.687543\end{array}$

C $\quad-2.687543 \quad-2.687543 \quad-2.687543$

$\begin{array}{llll}\mathrm{H} & -2.418574 & 1.170512 & 4.228595\end{array}$

$\mathrm{H} \quad 2.418574 \quad-1.170512 \quad 4.228595$

H $2.418574 \quad 1.170512 \quad-4.228595$

H $\quad-2.418574 \quad-1.170512 \quad-4.228595$

H $3.321597 \quad 3.321597 \quad-3.321597$

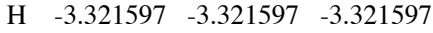

$\begin{array}{llll}\mathrm{H} & -3.321597 & 3.321597 & 3.321597\end{array}$

$\begin{array}{llll}\mathrm{H} & 3.321597 & -3.321597 & 3.321597\end{array}$ \begin{tabular}{|llll}
$\mathrm{C}$ & 0.897087 & 4.461957 & -0.897087
\end{tabular}

$\begin{array}{llll}\text { C } & -0.897087 & -4.461957 & -0.897087\end{array}$

$\begin{array}{llll}\text { C } & -0.897087 & 4.461957 & 0.897087\end{array}$

$\begin{array}{llll}\text { C } & 0.897087 & -4.461957 & 0.897087\end{array}$

$\begin{array}{lllll}\text { C } & 4.461957 & 0.897087 & -0.897087\end{array}$

$\begin{array}{llll}\text { C } & -4.461957 & -0.897087 & -0.897087\end{array}$

$\begin{array}{llll}\text { C } & -4.461957 & 0.897087 & 0.897087\end{array}$

$\begin{array}{llll}\text { C } & 4.461957 & -0.897087 & 0.897087\end{array}$

$\begin{array}{lllll}\text { C } & 0.897087 & 0.897087 & -4.461957\end{array}$

$\begin{array}{llll}\text { C } & -0.897087 & -0.897087 & -4.461957\end{array}$

$\begin{array}{llll}\text { C } & -0.897087 & 0.897087 & 4.461957\end{array}$

$\begin{array}{llll}\text { C } & 0.897087 & -0.897087 & 4.461957\end{array}$

$\begin{array}{llll}\mathrm{H} & 3.314241 & 3.314241 & -0.271068\end{array}$

H $\quad-3.314241 \quad-3.314241 \quad-0.271068$

$\begin{array}{llll}\mathrm{H} & -3.314241 & 3.314241 & 0.271068\end{array}$

$\begin{array}{llll}\mathrm{H} & 3.314241 & -3.314241 & 0.271068\end{array}$

$\begin{array}{llll}\mathrm{H} & -1.521359 & 3.311759 & -1.521359\end{array}$

$\begin{array}{llll}\mathrm{H} & 1.521359 & -3.311759 & -1.521359\end{array}$

$\begin{array}{llll}\mathrm{H} & 1.521359 & 3.311759 & 1.521359\end{array}$

$\begin{array}{llll}\mathrm{H} & -1.521359 & -3.311759 & 1.521359\end{array}$

$\begin{array}{llll}\mathrm{H} & 3.311759 & -1.521359 & -1.521359\end{array}$

$\begin{array}{llll}\mathrm{H} & -3.311759 & 1.521359 & -1.521359\end{array}$

$\begin{array}{llll}\mathrm{H} & -3.311759 & -1.521359 & 1.521359\end{array}$

$\begin{array}{llll}\mathrm{H} & 3.311759 & 1.521359 & 1.521359\end{array}$

$\begin{array}{llll}\mathrm{H} & -1.521359 & 1.521359 & -3.311759\end{array}$

$\begin{array}{llll}\mathrm{H} & 1.521359 & -1.521359 & -3.311759\end{array}$

$\begin{array}{llll}\mathrm{H} & 1.521359 & 1.521359 & 3.311759\end{array}$

$\begin{array}{llll}\mathrm{H} & -1.521359 & -1.521359 & 3.311759\end{array}$

$\begin{array}{llll}\mathrm{H} & 3.314241 & 0.271068 & -3.314241\end{array}$

$\begin{array}{llll}\mathrm{H} & -3.314241 & -0.271068 & -3.314241\end{array}$

$\begin{array}{llll}\mathrm{H} & -3.314241 & 0.271068 & 3.314241\end{array}$

$\begin{array}{llll}\text { H } & 3.314241 & -0.271068 & 3.314241\end{array}$

H $\quad 0.271068 \quad 3.314241 \quad-3.314241$

$\begin{array}{llll}\mathrm{H} & -0.271068 & -3.314241 & -3.314241\end{array}$

$\begin{array}{llll}\mathrm{H} & -0.271068 & 3.314241 & 3.314241\end{array}$

$\begin{array}{llll}\text { H } & 0.271068 & -3.314241 & 3.314241\end{array}$

$\begin{array}{llll}\text { C } & 4.469088 & 2.693421 & -2.693421\end{array}$

$\begin{array}{llll}\text { C } & -4.469088 & -2.693421 & -2.693421\end{array}$

$\begin{array}{llll}\text { C } & -4.469088 & 2.693421 & 2.693421\end{array}$

$\begin{array}{llll}\text { C } & 4.469088 & -2.693421 & 2.693421\end{array}$

$\begin{array}{llll}\text { C } & 2.693421 & 4.469088 & -2.693421\end{array}$

$\begin{array}{llll}\text { C } & -2.693421 & -4.469088 & -2.693421\end{array}$

$\begin{array}{llll}\text { C } & -2.693421 & 4.469088 & 2.693421\end{array}$

$\begin{array}{llll}\text { C } & 2.693421 & -4.469088 & 2.693421\end{array}$

$\begin{array}{lllll}\text { C } & 2.693421 & 2.693421 & -4.469088\end{array}$

$\begin{array}{llll}\text { C } & -2.693421 & -2.693421 & -4.469088\end{array}$

$\begin{array}{llll}\text { C } & -2.693421 & 2.693421 & 4.469088\end{array}$

$\begin{array}{llll}\text { C } & 2.693421 & -2.693421 & 4.469088\end{array}$

$\begin{array}{lllll}\mathrm{H} & 0.272628 & 5.120610 & -1.520127\end{array}$

$\begin{array}{llll}\mathrm{H} & -0.272628 & -5.120610 & -1.520127\end{array}$

$\begin{array}{llll}\mathrm{H} & -0.272628 & 5.120610 & 1.520127\end{array}$

$\begin{array}{llll}\mathrm{H} & 0.272628 & -5.120610 & 1.520127\end{array}$

$\begin{array}{llll}\mathrm{H} & 1.520127 & 5.120610 & -0.272628\end{array}$

$\begin{array}{llll}\mathrm{H} & -1.520127 & -5.120610 & -0.272628\end{array}$

$\begin{array}{llll}\mathrm{H} & -1.520127 & 5.120610 & 0.272628\end{array}$

$\begin{array}{llll}\mathrm{H} & 1.520127 & -5.120610 & 0.272628\end{array}$

$\begin{array}{llll}\text { H } & 5.120610 & 0.272628 & -1.520127\end{array}$

$\begin{array}{llll}\text { H } & -5.120610 & -0.272628 & -1.520127\end{array}$

$\begin{array}{llll}\mathrm{H} & -5.120610 & 0.272628 & 1.520127\end{array}$

$\begin{array}{llll}\text { H } & 5.120610 & -0.272628 & 1.520127\end{array}$

$\begin{array}{llll}\mathrm{H} & 5.120610 & 1.520127 & -0.272628\end{array}$

$\begin{array}{llll}\mathrm{H} & -5.120610 & -1.520127 & -0.272628\end{array}$

$\begin{array}{llll}\mathrm{H} & -5.120610 & 1.520127 & 0.272628\end{array}$

$\begin{array}{llll}\mathrm{H} & 5.120610 & -1.520127 & 0.272628\end{array}$

$\begin{array}{llll}\mathrm{H} & 0.272628 & 1.520127 & -5.120610\end{array}$

$\begin{array}{llll}\mathrm{H} & -0.272628 & -1.520127 & -5.120610\end{array}$

$\begin{array}{llll}\mathrm{H} & -0.272628 & 1.520127 & 5.120610\end{array}$

$\begin{array}{llll}\text { H } & 0.272628 & -1.520127 & 5.120610\end{array}$

$\begin{array}{llll}\mathrm{H} & 1.520127 & 0.272628 & -5.120610\end{array}$

$\begin{array}{llll}\mathrm{H} & -1.520127 & -0.272628 & -5.120610\end{array}$

$\begin{array}{llll}\mathrm{H} & -1.520127 & 0.272628 & 5.120610\end{array}$ 
\begin{tabular}{|llll}
$\mathrm{H}$ & 1.520127 & -0.272628 & 5.120610
\end{tabular}

H $2.067805 \quad 5.125896 \quad-3.315593$

$\begin{array}{llll}\mathrm{H} & -2.067805 & -5.125896 & -3.315593\end{array}$

H $\quad-2.067805 \quad 5.125896 \quad 3.315593$

$\begin{array}{llll}\mathrm{H} & 2.067805 & -5.125896 & 3.315593\end{array}$

H $3.315593 \quad 5.125896 \quad-2.067805$

$\begin{array}{llll}\mathrm{H} & -3.315593 & -5.125896 & -2.067805\end{array}$

$\begin{array}{llll}\mathrm{H} & -3.315593 & 5.125896 & 2.067805\end{array}$

$\begin{array}{llll}\mathrm{H} & 3.315593 & -5.125896 & 2.067805\end{array}$

$\begin{array}{llll}\mathrm{H} & 5.125896 & 2.067805 & -3.315593\end{array}$

H $\quad-5.125896 \quad-2.067805 \quad-3.315593$

$\begin{array}{llll}\mathrm{H} & -5.125896 & 2.067805 & 3.315593\end{array}$

H $\quad 5.125896 \quad-2.067805 \quad 3.315593$

$\begin{array}{llll}\mathrm{H} & 5.125896 & 3.315593 & -2.067805\end{array}$

$\begin{array}{llll}\mathrm{H} & -5.125896 & -3.315593 & -2.067805\end{array}$

$\begin{array}{llll}\mathrm{H} & -5.125896 & 3.315593 & 2.067805\end{array}$

$\begin{array}{llll}\mathrm{H} & 5.125896 & -3.315593 & 2.067805\end{array}$

H $2.067805 \quad 3.315593 \quad-5.125896$

$\begin{array}{llll}\mathrm{H} & -2.067805 & -3.315593 & -5.125896\end{array}$

$\begin{array}{llll}\mathrm{H} & -2.067805 & 3.315593 & 5.125896\end{array}$

$\begin{array}{llll}\mathrm{H} & 2.067805 & -3.315593 & 5.125896\end{array}$

$\begin{array}{llll}\text { C } & 3.584398 & 3.584398 & -3.584398\end{array}$

$\begin{array}{llll}\text { C } & -3.584398 & -3.584398 & -3.584398\end{array}$

$\begin{array}{llll}\text { C } & -3.584398 & 3.584398 & 3.584398\end{array}$

$\begin{array}{llll}\text { C } & 3.584398 & -3.584398 & 3.584398\end{array}$

$\begin{array}{llll}\mathrm{H} & 3.315593 & 2.067805 & -5.125896\end{array}$

$\begin{array}{llll}\mathrm{H} & -3.315593 & -2.067805 & -5.125896\end{array}$

$\begin{array}{llll}\mathrm{H} & -3.315593 & 2.067805 & 5.125896\end{array}$

$\begin{array}{llll}\mathrm{H} & 3.315593 & -2.067805 & 5.125896\end{array}$

$\begin{array}{llll}\mathrm{H} & 4.218394 & 4.218394 & -4.218394\end{array}$

H $\quad-4.218394 \quad-4.218394 \quad-4.218394$

$\begin{array}{llll}\mathrm{H} & -4.218394 & 4.218394 & 4.218394\end{array}$

H $\quad 4.218394 \quad-4.218394 \quad 4.218394$

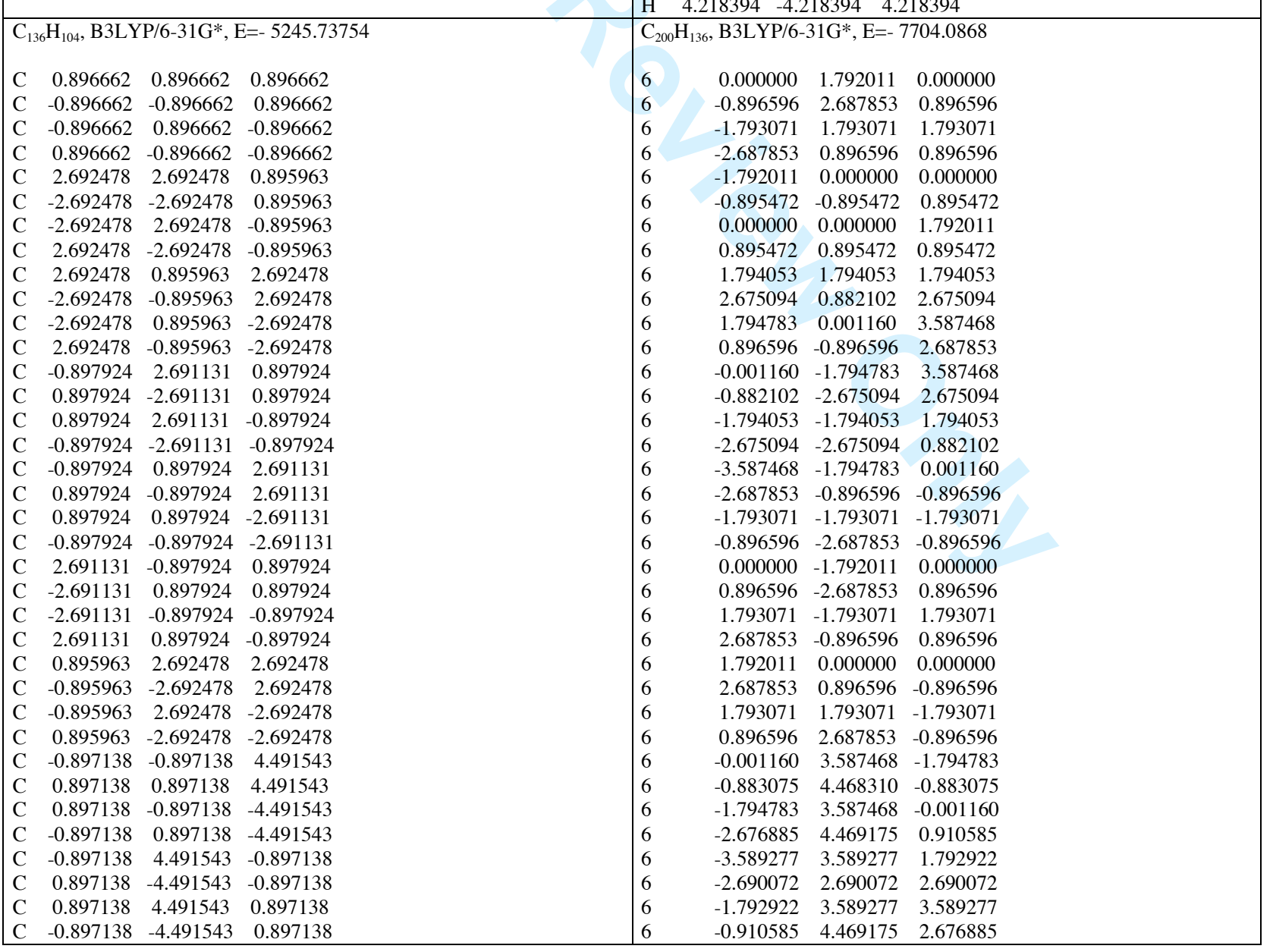

URL: http://mc.manuscriptcentral.com/tandf/tmph 
$\begin{array}{lll}0.001160 & 3.587468 & 1.794783\end{array}$

$\begin{array}{llll}0.883075 & 4.468310 & 0.883075\end{array}$

$\begin{array}{llll}1.794783 & 3.587468 & 0.001160\end{array}$

$\begin{array}{llll}2.675094 & 2.675094 & 0.882102\end{array}$

$\begin{array}{lll}3.587468 & 1.794783 & 0.001160\end{array}$

$\begin{array}{llll}4.469175 & 2.676885 & -0.910585\end{array}$

$\begin{array}{llll}3.589277 & 3.589277 & -1.792922\end{array}$

$\begin{array}{llll}2.676885 & 4.469175 & -0.910585\end{array}$

$\begin{array}{llll}1.793894 & 5.387827 & -1.793894\end{array}$

$\begin{array}{llll}0.910585 & 4.469175 & -2.676885\end{array}$

$\begin{array}{llll}1.792922 & 3.589277 & -3.589277\end{array}$

$\begin{array}{llll}2.690072 & 2.690072 & -2.690072\end{array}$

$\begin{array}{llll}3.589277 & 1.792922 & -3.589277\end{array}$

$\begin{array}{llll}2.676885 & 0.910585 & -4.469175\end{array}$

$\begin{array}{llll}1.793894 & 1.793894 & -5.387827\end{array}$

$\begin{array}{llll}0.910585 & 2.676885 & -4.469175\end{array}$

$\begin{array}{llll}-0.001160 & 1.794783 & -3.587468\end{array}$

$\begin{array}{lll}-0.882102 & 2.675094 & -2.675094\end{array}$

$\begin{array}{llll}-1.794053 & 1.794053 & -1.794053\end{array}$

$\begin{array}{llll}-0.895472 & 0.895472 & -0.895472\end{array}$

$\begin{array}{llll}0.000000 & 0.000000 & -1.792011\end{array}$

$\begin{array}{llll}0.895472 & -0.895472 & -0.895472\end{array}$

$\begin{array}{llll}1.794053 & -1.794053 & -1.794053\end{array}$

$\begin{array}{llll}2.675094 & -0.882102 & -2.675094\end{array}$

$3.587468-0.001160 \quad-1.794783$

$\begin{array}{llll}4.469175 & 0.910585 & -2.676885\end{array}$

$\begin{array}{llll}5.387827 & 1.793894 & -1.793894\end{array}$

$\begin{array}{llll}6.253308 & 0.896782 & -0.896782\end{array}$

$\begin{array}{llll}6.911662 & 0.272674 & -1.520400\end{array}$

$\begin{array}{llll}6.911662 & 1.520400 & -0.272674\end{array}$

$\begin{array}{llll}5.387019 & 0.000000 & 0.000000\end{array}$

$\begin{array}{llll}4.468310 & 0.883075 & 0.883075\end{array}$

$\begin{array}{lll}3.587468 & 0.001160 & 1.794783\end{array}$

$\begin{array}{llll}4.469175 & -0.910585 & 2.676885\end{array}$

$\begin{array}{llll}3.589277 & -1.792922 & 3.589277\end{array}$

$\begin{array}{llll}2.676885 & -0.910585 & 4.469175\end{array}$

$\begin{array}{lll}1.793894 & -1.793894 & 5.387827\end{array}$

$\begin{array}{lll}0.896782 & -0.896782 & 6.253308\end{array}$

$\begin{array}{lll}0.000000 & 0.000000 & 5.387019\end{array}$

$\begin{array}{lll}-0.896782 & 0.896782 & 6.253308\end{array}$

$\begin{array}{lll}-1.793894 & 1.793894 & 5.387827\end{array}$

$\begin{array}{lll}-0.910585 & 2.676885 & 4.469175\end{array}$

$\begin{array}{lll}0.001160 & 1.794783 & 3.587468\end{array}$

$\begin{array}{lll}-0.896596 & 0.896596 & 2.687853\end{array}$

$\begin{array}{lll}-1.794783 & -0.001160 & 3.587468\end{array}$

$\begin{array}{lll}-2.676885 & 0.910585 & 4.469175\end{array}$

$\begin{array}{lll}-3.589277 & 1.792922 & 3.589277\end{array}$

$\begin{array}{lll}-4.469175 & 0.910585 & 2.676885\end{array}$

$\begin{array}{llll}-5.387827 & 1.793894 & 1.793894\end{array}$

$\begin{array}{lll}-4.469175 & 2.676885 & 0.910585\end{array}$

$\begin{array}{llll}-3.587468 & 1.794783 & -0.001160\end{array}$

$\begin{array}{lll}-4.468310 & 0.883075 & -0.883075\end{array}$

$\begin{array}{llll}-3.587468 & 0.001160 & -1.794783\end{array}$

$\begin{array}{lll}-2.675094 & 0.882102 & -2.675094\end{array}$

$\begin{array}{llll}-1.794783 & 0.001160 & -3.587468\end{array}$

$-2.676885-0.910585-4.469175$

$\begin{array}{llll}-1.793894 & -1.793894 & -5.387827\end{array}$

$-0.910585-2.676885-4.469175$

$\begin{array}{llll}-0.271610 & -3.314221 & -5.103817\end{array}$ $\begin{array}{lll}-1.792922 & -3.589277 & -3.589277\end{array}$

$\begin{array}{llll}-0.910585 & -4.469175 & -2.676885\end{array}$

$\begin{array}{lll}0.001160 & -3.587468 & -1.794783\end{array}$

$\begin{array}{llll}0.882102 & -2.675094 & -2.675094\end{array}$

$\begin{array}{llll}0.001160 & -1.794783 & -3.587468\end{array}$

$\begin{array}{llll}0.883075 & -0.883075 & -4.468310\end{array}$

$\begin{array}{lll}1.794783 & -0.001160 & -3.587468\end{array}$

$\begin{array}{llll}0.896596 & 0.896596 & -2.687853\end{array}$

$\begin{array}{llll}0.000000 & 0.000000 & -5.387019\end{array}$

$\begin{array}{llll}-0.883075 & 0.883075 & -4.468310\end{array}$

$\begin{array}{llll}-1.521319 & 1.521319 & -5.102823\end{array}$

$\begin{array}{llll}-0.896782 & -0.896782 & -6.253308\end{array}$ 
$\begin{array}{lll}-0.272674 & -1.520400 & -6.911662\end{array}$ $\begin{array}{llll}-1.520400 & -0.272674 & -6.911662\end{array}$ $\begin{array}{ccc}0.896782 & 0.896782 & -6.253308\end{array}$ $\begin{array}{llll}1.520400 & 0.272674 & -6.911662\end{array}$ $\begin{array}{llll}0.272674 & 1.520400 & -6.911662\end{array}$ $\begin{array}{llll}1.521319 & -1.521319 & -5.102823\end{array}$ $\begin{array}{llll}-0.896596 & -0.896596 & -2.687853\end{array}$ $\begin{array}{llll}1.519903 & -3.311870 & -3.311870\end{array}$ $\begin{array}{llll}0.883075 & -4.468310 & -0.883075\end{array}$ $\begin{array}{llll}1.794783 & -3.587468 & -0.001160\end{array}$ $\begin{array}{llll}2.676885 & -4.469175 & 0.910585\end{array}$ $\begin{array}{lll}1.793894 & -5.387827 & 1.793894\end{array}$ $\begin{array}{llll}2.690652 & -6.255059 & 2.690652\end{array}$ $\begin{array}{llll}3.589189 & -5.391877 & 3.589189\end{array}$ $\begin{array}{llll}4.472447 & -4.472447 & 2.704829\end{array}$ $\begin{array}{lll}5.391877 & -3.589189 & 3.589189\end{array}$ $\begin{array}{llll}6.255059 & -2.690652 & 2.690652\end{array}$ $\begin{array}{lll}5.387827 & -1.793894 & 1.793894\end{array}$ $\begin{array}{llll}4.469175 & -2.676885 & 0.910585\end{array}$ $\begin{array}{llll}3.589277 & -3.589277 & 1.792922\end{array}$ $\begin{array}{llll}2.690072 & -2.690072 & 2.690072\end{array}$ $\begin{array}{lll}1.792922 & -3.589277 & 3.589277\end{array}$ $\begin{array}{llll}0.910585 & -4.469175 & 2.676885\end{array}$ $\begin{array}{lll}-0.001160 & -3.587468 & 1.794783\end{array}$ $\begin{array}{lll}-0.883075 & -4.468310 & 0.883075\end{array}$ $\begin{array}{lll}-1.794783 & -3.587468 & 0.001160\end{array}$ $\begin{array}{llll}-2.676885 & -4.469175 & -0.910585\end{array}$ $\begin{array}{llll}-3.589277 & -3.589277 & -1.792922\end{array}$ $\begin{array}{llll}-4.469175 & -2.676885 & -0.910585\end{array}$ $\begin{array}{llll}-5.387827 & -1.793894 & -1.793894\end{array}$ $\begin{array}{llll}-4.469175 & -0.910585 & -2.676885\end{array}$ $\begin{array}{llll}-3.589277 & -1.792922 & -3.589277\end{array}$ $\begin{array}{lll}-2.690072 & -2.690072 & -2.690072\end{array}$ $\begin{array}{llll}-4.472447 & -2.704829 & -4.472447\end{array}$ $\begin{array}{llll}-5.391877 & -3.589189 & -3.589189\end{array}$ $\begin{array}{llll}-4.472447 & -4.472447 & -2.704829\end{array}$ $\begin{array}{lll}-3.589189 & -5.391877 & -3.589189\end{array}$ $\begin{array}{llll}-2.690652 & -6.255059 & -2.690652\end{array}$ $\begin{array}{llll}-1.793894 & -5.387827 & -1.793894\end{array}$ $\begin{array}{llll}-0.896782 & -6.253308 & -0.896782\end{array}$ $\begin{array}{lll}0.000000 & -5.387019 & 0.000000\end{array}$ $\begin{array}{llll}0.896782 & -6.253308 & 0.896782\end{array}$ $\begin{array}{llll}1.520400 & -6.911662 & 0.272674\end{array}$ $\begin{array}{lll}0.272674 & -6.911662 & 1.520400\end{array}$ $\begin{array}{llll}-0.272674 & -6.911662 & -1.520400\end{array}$ $\begin{array}{llll}-1.520400 & -6.911662 & -0.272674\end{array}$ $\begin{array}{llll}-2.065823 & -6.913573 & -3.313438\end{array}$ $\begin{array}{llll}-3.313438 & -6.913573 & -2.065823\end{array}$ $\begin{array}{llll}-2.704829 & -4.472447 & -4.472447\end{array}$ $\begin{array}{llll}-3.589189 & -3.589189 & -5.391877\end{array}$ $\begin{array}{llll}-4.487031 & -4.487031 & -6.262723\end{array}$ $\begin{array}{lll}-5.378061 & -5.378061 & -5.378061\end{array}$ $\begin{array}{llll}-6.262723 & -4.487031 & -4.487031\end{array}$ $\begin{array}{lll}-6.919385 & -3.861222 & -5.109127\end{array}$ $\begin{array}{lll}-6.919385 & -5.109127 & -3.861222\end{array}$ $\begin{array}{llll}-4.487031 & -6.262723 & -4.487031\end{array}$ $\begin{array}{lll}-3.861222 & -6.919385 & -5.109127\end{array}$ $\begin{array}{llll}-5.109127 & -6.919385 & -3.861222\end{array}$ $\begin{array}{lll}-6.012014 & -6.012014 & -6.012014\end{array}$ $\begin{array}{lll}-3.861222 & -5.109127 & -6.919385\end{array}$ $\begin{array}{lll}-5.109127 & -3.861222 & -6.919385\end{array}$ $\begin{array}{lll}-2.690652 & -2.690652 & -6.255059\end{array}$ $-2.065823-3.313438-6.913573$ $\begin{array}{lll}-3.313438 & -2.065823 & -6.913573\end{array}$ $-2.064503 \quad-5.107540 \quad-5.107540$ $\begin{array}{lll}-5.107540 & -5.107540 & -2.064503\end{array}$ $\begin{array}{lll}-6.255059 & -2.690652 & -2.690652\end{array}$ $\begin{array}{lll}-6.913573 & -3.313438 & -2.065823\end{array}$ $-6.913573-2.065823-3.313438$ $\begin{array}{lll}-5.107540 & -2.064503 & -5.107540\end{array}$ $\begin{array}{lll}-5.103817 & -0.271610 & -3.314221\end{array}$ 
2

3

4

5

6

7

8

9

10

11

12

13

14

15

16

17

18

19

20

21

22

23

24

25

26

27

28

29

30

31

32

33

34

35

36

37

38

39

40

41

42

43

44

45

46

47

48

49

50

51

52

53

54

55

56

57

58

59

60 $\begin{array}{lll}-6.253308 & -0.896782 & -0.896782\end{array}$

$\begin{array}{lll}-5.387019 & 0.000000 & 0.000000\end{array}$

$\begin{array}{lll}-4.468310 & -0.883075 & 0.883075\end{array}$

$\begin{array}{lll}-3.587468 & -0.001160 & 1.794783\end{array}$

$\begin{array}{lll}-2.675094 & -0.882102 & 2.675094\end{array}$

$\begin{array}{lll}-3.311870 & -1.519903 & 3.311870\end{array}$

$\begin{array}{lll}-5.102823 & -1.521319 & 1.521319\end{array}$

$\begin{array}{lll}-6.253308 & 0.896782 & 0.896782\end{array}$

$\begin{array}{lll}-6.911662 & 0.272674 & 1.520400\end{array}$

$\begin{array}{lll}-6.911662 & 1.520400 & 0.272674\end{array}$

$\begin{array}{llll}-6.911662 & -1.520400 & -0.272674\end{array}$

$\begin{array}{llll}-6.911662 & -0.272674 & -1.520400\end{array}$

$\begin{array}{lll}-5.103817 & -3.314221 & -0.271610\end{array}$

$\begin{array}{lll}-3.314221 & -5.103817 & -0.271610\end{array}$

$\begin{array}{lll}-1.521319 & -5.102823 & 1.521319\end{array}$

$\begin{array}{lll}0.271610 & -5.103817 & 3.314221\end{array}$

$\begin{array}{llll}2.704829 & -4.472447 & 4.472447\end{array}$

$\begin{array}{lll}3.589189 & -3.589189 & 5.391877\end{array}$

$\begin{array}{llll}4.472447 & -2.704829 & 4.472447\end{array}$

$\begin{array}{lll}5.107540 & -2.064503 & 5.107540\end{array}$

$\begin{array}{llll}2.690652 & -2.690652 & 6.255059\end{array}$

$\begin{array}{lll}3.313438 & -2.065823 & 6.913573\end{array}$

$\begin{array}{lll}2.065823 & -3.313438 & 6.913573\end{array}$

$\begin{array}{llll}4.487031 & -4.487031 & 6.262723\end{array}$

$\begin{array}{lll}5.109127 & -3.861222 & 6.919385\end{array}$

$\begin{array}{lll}3.861222 & -5.109127 & 6.919385\end{array}$

$\begin{array}{llll}5.378061 & -5.378061 & 5.378061\end{array}$

$\begin{array}{lll}4.487031 & -6.262723 & 4.487031\end{array}$

$\begin{array}{lll}5.109127 & -6.919385 & 3.861222\end{array}$

$\begin{array}{lll}3.861222 & -6.919385 & 5.109127\end{array}$

$\begin{array}{llll}6.262723 & -4.487031 & 4.487031\end{array}$

$\begin{array}{lll}6.919385 & -5.109127 & 3.861222\end{array}$

$\begin{array}{lll}6.919385 & -3.861222 & 5.109127\end{array}$

$\begin{array}{lll}6.012014 & -6.012014 & 6.012014\end{array}$

$\begin{array}{lll}2.064503 & -5.107540 & 5.107540\end{array}$

$\begin{array}{llll}0.910585 & -2.676885 & 4.469175\end{array}$

$\begin{array}{lll}0.271610 & -3.314221 & 5.103817\end{array}$

$\begin{array}{llll}3.587468 & -1.794783 & -0.001160\end{array}$

$\begin{array}{llll}2.675094 & -2.675094 & -0.882102\end{array}$

$\begin{array}{llll}3.311870 & -3.311870 & -1.519903\end{array}$

$\begin{array}{lll}4.468310 & -0.883075 & -0.883075\end{array}$

$\begin{array}{llll}5.102823 & -1.521319 & -1.521319\end{array}$

$\begin{array}{llll}5.103817 & -3.314221 & 0.271610\end{array}$

$\begin{array}{llll}6.253308 & -0.896782 & 0.896782\end{array}$

$\begin{array}{llll}6.911662 & -1.520400 & 0.272674\end{array}$

$\begin{array}{lll}6.911662 & -0.272674 & 1.520400\end{array}$

$\begin{array}{llll}6.913573 & -2.065823 & 3.313438\end{array}$

$\begin{array}{lll}6.913573 & -3.313438 & 2.065823\end{array}$

$\begin{array}{lll}5.107540 & -5.107540 & 2.064503\end{array}$

$\begin{array}{lll}3.313438 & -6.913573 & 2.065823\end{array}$

$\begin{array}{llll}2.065823 & -6.913573 & 3.313438\end{array}$

$\begin{array}{llll}3.314221 & -5.103817 & 0.271610\end{array}$

$\begin{array}{llll}1.521319 & -5.102823 & -1.521319\end{array}$

$\begin{array}{llll}-0.271610 & -5.103817 & -3.314221\end{array}$

$\begin{array}{lll}-3.314221 & -0.271610 & -5.103817\end{array}$

$\begin{array}{llll}-3.311870 & 1.519903 & -3.311870\end{array}$

$\begin{array}{llll}-5.102823 & 1.521319 & -1.521319\end{array}$

$\begin{array}{llll}-2.675094 & 2.675094 & -0.882102\end{array}$

$\begin{array}{llll}-3.311870 & 3.311870 & -1.519903\end{array}$

$\begin{array}{lll}-5.103817 & 3.314221 & 0.271610\end{array}$

$\begin{array}{lll}-6.255059 & 2.690652 & 2.690652\end{array}$

$\begin{array}{lll}-5.391877 & 3.589189 & 3.589189\end{array}$

$\begin{array}{lll}-4.472447 & 2.704829 & 4.472447\end{array}$

$\begin{array}{lll}-3.589189 & 3.589189 & 5.391877\end{array}$

$\begin{array}{lll}-2.690652 & 2.690652 & 6.255059\end{array}$

$\begin{array}{lll}-3.313438 & 2.065823 & 6.913573\end{array}$

$\begin{array}{lll}-2.065823 & 3.313438 & 6.913573\end{array}$

$\begin{array}{lll}-2.704829 & 4.472447 & 4.472447\end{array}$

$\begin{array}{lll}-3.589189 & 5.391877 & 3.589189\end{array}$

$\begin{array}{lll}-4.472447 & 4.472447 & 2.704829\end{array}$

$\begin{array}{lll}-5.107540 & 5.107540 & 2.064503\end{array}$ 


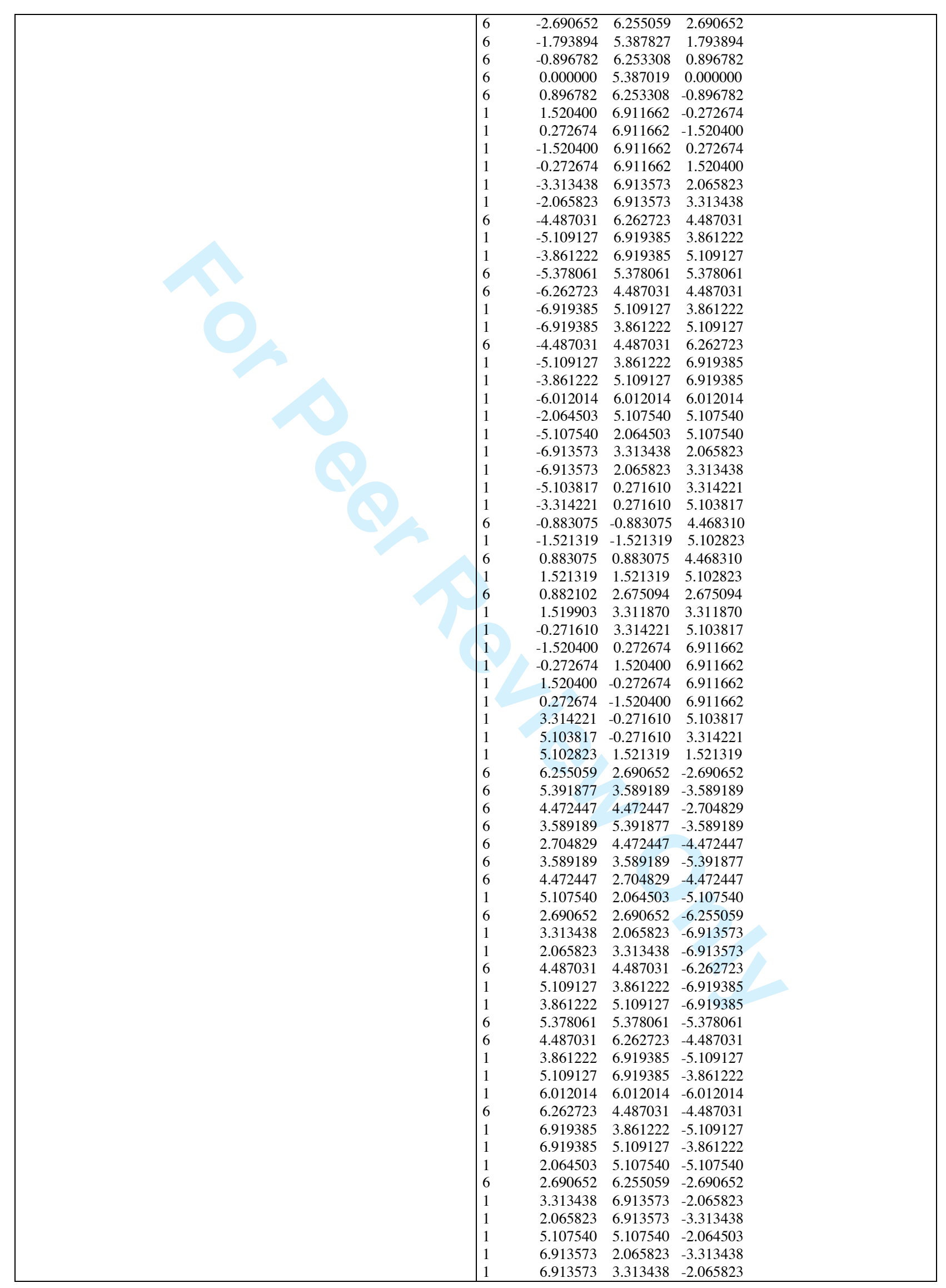


2

3

4

5

6

7

8

9

10

11

12

13

14

15

16

17

18

19

20

21

22

23

24

25

26

27

28

29

30

31

32

33

34

35

36

37

38

39

40

41

42

43

44

45

46

47

48

49

50

51

52

53

54

55

56

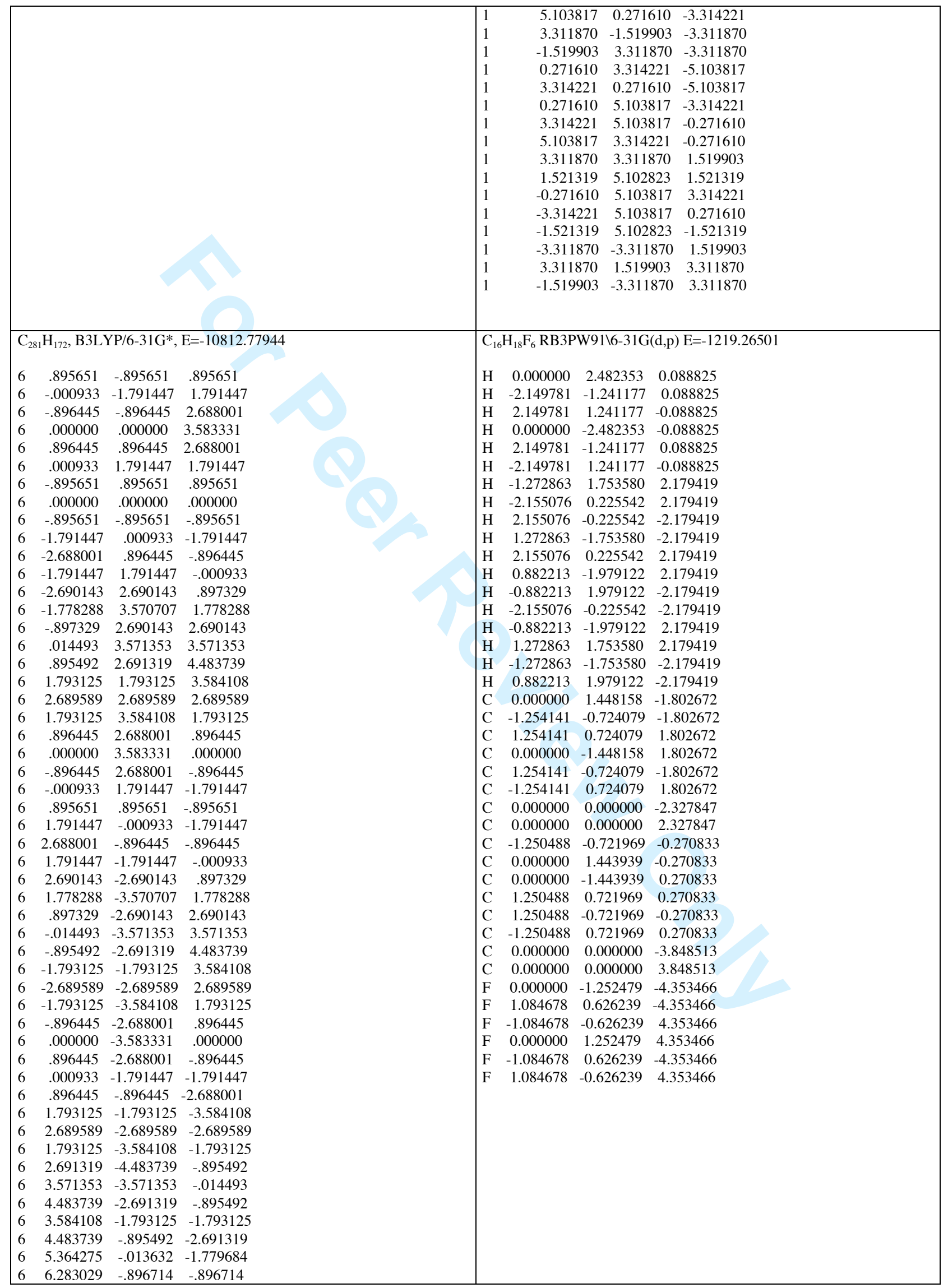

URL: http://mc.manuscriptcentral.com/tandf/tmph 
2

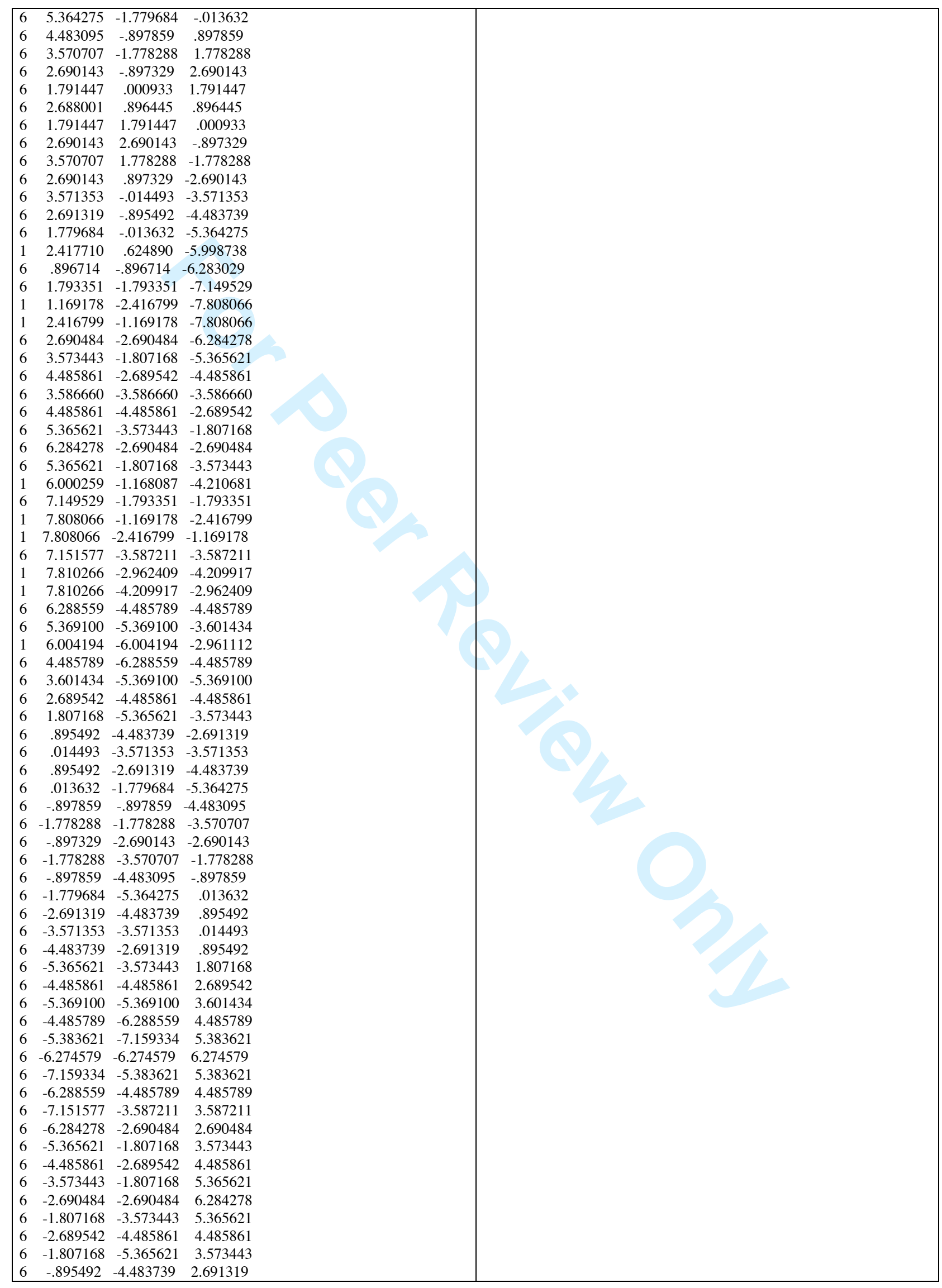


\begin{tabular}{llll}
\hline 6 & -.013632 & -5.364275 & 1.779684
\end{tabular}

$\begin{array}{lllll}6 & .897859 & -4.483095 & .897859\end{array}$

$\begin{array}{lllll}6 & 1.779684 & -5.364275 & -.013632\end{array}$

$\begin{array}{lllll}6 & .896714 & -6.283029 & -.896714\end{array}$

$\begin{array}{lllll}6 & 1.793351 & -7.149529 & -1.793351\end{array}$

$\begin{array}{lllll}6 & 2.690484 & -6.284278 & -2.690484\end{array}$

$\begin{array}{lllll}6 & 3.573443 & -5.365621 & -1.807168\end{array}$

$\begin{array}{llll}1 & 4.210681 & -6.000259 & -1.168087\end{array}$

$\begin{array}{lllll}6 & 3.587211 & -7.151577 & -3.587211\end{array}$

$\begin{array}{llll}1 & 2.962409 & -7.810266 & -4.209917\end{array}$

$\begin{array}{lllll}1 & 4.209917 & -7.810266 & -2.962409\end{array}$

$\begin{array}{lllll}1 & 1.169178 & -7.808066 & -2.416799\end{array}$

$\begin{array}{lllll}1 & 2.416799 & -7.808066 & -1.169178\end{array}$

$\begin{array}{lllll}6 & .013632 & -5.364275 & -1.779684\end{array}$

$\begin{array}{lllll}1 & -.624890 & -5.998738 & -2.417710\end{array}$

$\begin{array}{lllll}6 & .000000 & -7.148945 & .000000\end{array}$

$\begin{array}{llll}6 & -.896714 & -6.283029 & .896714\end{array}$

$\begin{array}{llll}6 & -1.793351 & -7.149529 & 1.793351\end{array}$

$\begin{array}{lllll}1 & -2.416799 & -7.808066 & 1.169178\end{array}$

$\begin{array}{lllll}1 & -1.169178 & -7.808066 & 2.416799\end{array}$

$\begin{array}{lllll}6 & -2.690484 & -6.284278 & 2.690484\end{array}$

$\begin{array}{llll}6 & -3.573443 & -5.365621 & 1.807168\end{array}$

$\begin{array}{lllll}1 & -4.210681 & -6.000259 & 1.168087\end{array}$

$\begin{array}{llll}6 & -3.587211 & -7.151577 & 3.587211\end{array}$

$\begin{array}{lllll}1 & -4.209917 & -7.810266 & 2.962409\end{array}$

$\begin{array}{lllll}1 & -2.962409 & -7.810266 & 4.209917\end{array}$

$\begin{array}{lllll}1 & -.623818 & -7.807467 & -.623818\end{array}$

$\begin{array}{lllll}1 & .623818 & -7.807467 & .623818\end{array}$

$\begin{array}{lllll}1 & 2.417710 & -5.998738 & .624890\end{array}$

$\begin{array}{lllll}1 & .624890 & -5.998738 & 2.417710\end{array}$

$\begin{array}{llll}1 & -1.168087 & -6.000259 & 4.210681\end{array}$

$\begin{array}{lllll}6 & -3.586660 & -3.586660 & 3.586660\end{array}$

$\begin{array}{llll}6 & -3.601434 & -5.369100 & 5.369100\end{array}$

$\begin{array}{lllll}1 & -2.961112 & -6.004194 & 6.004194\end{array}$

$\begin{array}{llll}6 & -4.485789 & -4.485789 & 6.288559\end{array}$

$\begin{array}{lllll}6 & -5.369100 & -3.601434 & 5.369100\end{array}$

$\begin{array}{lllll}1 & -6.004194 & -2.961112 & 6.004194\end{array}$

$\begin{array}{lllll}6 & -3.587211 & -3.587211 & 7.151577\end{array}$

$\begin{array}{llll}1 & -2.962409 & -4.209917 & 7.810266\end{array}$

$\begin{array}{lllll}1 & -4.209917 & -2.962409 & 7.810266\end{array}$

$\begin{array}{llll}6 & -5.383621 & -5.383621 & 7.159334\end{array}$

$\begin{array}{lllll}1 & -4.758027 & -6.005813 & 7.816194\end{array}$

$\begin{array}{llll}1 & -6.005813 & -4.758027 & 7.816194\end{array}$

$\begin{array}{lllll}1 & -1.168087 & -4.210681 & 6.000259\end{array}$

$\begin{array}{llll}6 & -1.793351 & -1.793351 & 7.149529\end{array}$

$\begin{array}{lllll}6 & -.896714 & -.896714 & 6.283029\end{array}$

$\begin{array}{lllll}6 & -1.779684 & -.013632 & 5.364275\end{array}$

$\begin{array}{lllll}6 & -2.691319 & -.895492 & 4.483739\end{array}$

$\begin{array}{llll}6 & -3.571353 & -.014493 & 3.571353\end{array}$

$\begin{array}{llll}6 & -2.690143 & .897329 & 2.690143\end{array}$

$\begin{array}{llll}6 & -1.791447 & -.000933 & 1.791447\end{array}$

$\begin{array}{lllll}6 & -2.688001 & -.896445 & .896445\end{array}$

$\begin{array}{llll}6 & -3.584108 & -1.793125 & 1.793125\end{array}$

$\begin{array}{llll}6 & -4.483739 & -.895492 & 2.691319\end{array}$

$\begin{array}{llll}6 & -5.364275 & -.013632 & 1.779684\end{array}$

$\begin{array}{llll}6 & -4.483095 & .897859 & .897859\end{array}$

$\begin{array}{lllll}6 & -5.364275 & 1.779684 & -.013632\end{array}$

$\begin{array}{llll}6 & -4.483739 & 2.691319 & -.895492\end{array}$

$\begin{array}{lllll}6 & -3.584108 & 1.793125 & -1.793125\end{array}$

$\begin{array}{llll}6 & -2.689589 & 2.689589 & -2.689589\end{array}$

$\begin{array}{lllll}6 & -1.793125 & 1.793125 & -3.584108\end{array}$

$\begin{array}{llll}6 & -.896445 & .896445 & -2.688001\end{array}$

$\begin{array}{lllll}6 & .000000 & .000000 & -3.583331\end{array}$

$\begin{array}{lllll}6 & .897859 & .897859 & -4.483095\end{array}$

$\begin{array}{lllll}6 & 1.778288 & 1.778288 & -3.570707\end{array}$

$\begin{array}{lllll}6 & .897329 & 2.690143 & -2.690143\end{array}$

$\begin{array}{lllll}6 & -.014493 & 3.571353 & -3.571353\end{array}$

$\begin{array}{lllll}6 & -.895492 & 4.483739 & -2.691319\end{array}$

$\begin{array}{lllll}6 & -1.793125 & 3.584108 & -1.793125\end{array}$

$\begin{array}{lllll}6 & -2.691319 & 4.483739 & -.895492\end{array}$

$\begin{array}{lllll}6 & -3.573443 & 5.365621 & -1.807168\end{array}$ 
\begin{tabular}{lllll}
\hline 6 & -4.485861 & 4.485861 & -2.689542
\end{tabular}

$\begin{array}{lllll}6 & -3.586660 & 3.586660 & -3.586660\end{array}$

$\begin{array}{lllll}6 & -4.485861 & 2.689542 & -4.485861\end{array}$

$\begin{array}{lllll}6 & -5.369100 & 3.601434 & -5.369100\end{array}$

$\begin{array}{lllll}6 & -6.288559 & 4.485789 & -4.485789\end{array}$

$\begin{array}{llll}6 & -7.151577 & 3.587211 & -3.587211\end{array}$

$\begin{array}{lllll}6 & -6.284278 & 2.690484 & -2.690484\end{array}$

$\begin{array}{llll}6 & -5.365621 & 1.807168 & -3.573443\end{array}$

$\begin{array}{lllll}6 & -4.483739 & .895492 & -2.691319\end{array}$

$\begin{array}{llll}6 & -5.364275 & .013632 & -1.779684\end{array}$

$\begin{array}{lllll}6 & -6.283029 & .896714 & -.896714\end{array}$

$\begin{array}{llll}6 & -7.148945 & .000000 & .000000\end{array}$

$\begin{array}{llll}6 & -6.283029 & -.896714 & .896714\end{array}$

$\begin{array}{llll}6 & -5.364275 & -1.779684 & .013632\end{array}$

$\begin{array}{lllll}6 & -4.483095 & -.897859 & -.897859\end{array}$

$\begin{array}{llll}6 & -3.570707 & -1.778288 & -1.778288\end{array}$

$\begin{array}{llll}6 & -2.690143 & -.897329 & -2.690143\end{array}$

$\begin{array}{llll}6 & -3.571353 & .014493 & -3.571353\end{array}$

$\begin{array}{lllll}6 & -2.691319 & .895492 & -4.483739\end{array}$

$\begin{array}{lllll}6 & -1.779684 & .013632 & -5.364275\end{array}$

$\begin{array}{lllll}6 & -.896714 & .896714 & -6.283029\end{array}$

$\begin{array}{lllll}6 & -.013632 & 1.779684 & -5.364275\end{array}$

$\begin{array}{lllll}6 & -.895492 & 2.691319 & -4.483739\end{array}$

$\begin{array}{llll}6 & -1.807168 & 3.573443 & -5.365621\end{array}$

$\begin{array}{lllll}6 & -2.689542 & 4.485861 & -4.485861\end{array}$

$\begin{array}{lllll}6 & -1.807168 & 5.365621 & -3.573443\end{array}$

$\begin{array}{lllll}6 & -2.690484 & 6.284278 & -2.690484\end{array}$

$\begin{array}{lllll}6 & -1.793351 & 7.149529 & -1.793351\end{array}$

$\begin{array}{llll}6 & -.896714 & 6.283029 & -.896714\end{array}$

$\begin{array}{lllll}6 & -.013632 & 5.364275 & -1.779684\end{array}$

$\begin{array}{llll}6 & .897859 & 4.483095 & -.897859\end{array}$

$\begin{array}{lllll}6 & 1.779684 & 5.364275 & .013632\end{array}$

$\begin{array}{llll}6 & 2.691319 & 4.483739 & .895492\end{array}$

$\begin{array}{llll}6 & 3.573443 & 5.365621 & 1.807168\end{array}$

$\begin{array}{llll}6 & 4.485861 & 4.485861 & 2.689542\end{array}$

$\begin{array}{llll}6 & 5.365621 & 3.573443 & 1.807168\end{array}$

$\begin{array}{llll}6 & 6.284278 & 2.690484 & 2.690484\end{array}$

$\begin{array}{llll}6 & 5.365621 & 1.807168 & 3.573443\end{array}$

$\begin{array}{llll}6 & 4.483739 & .895492 & 2.691319\end{array}$

$\begin{array}{lllll}6 & 3.571353 & .014493 & 3.571353\end{array}$

$\begin{array}{lllll}6 & 2.691319 & .895492 & 4.483739\end{array}$

$\begin{array}{llll}6 & 1.779684 & .013632 & 5.364275\end{array}$

$\begin{array}{llll}6 & .897859 & -.897859 & 4.483095\end{array}$

$\begin{array}{lllll}6 & -.013632 & -1.779684 & 5.364275\end{array}$

$\begin{array}{lllll}1 & .624890 & -2.417710 & 5.998738\end{array}$

$\begin{array}{llll}6 & 1.778288 & -1.778288 & 3.570707\end{array}$

$\begin{array}{lllll}1 & 2.415747 & -2.415747 & 4.207200\end{array}$

$\begin{array}{llll}6 & .896714 & .896714 & 6.283029\end{array}$

$\begin{array}{llll}6 & 1.793351 & 1.793351 & 7.149529\end{array}$

$\begin{array}{llll}6 & 2.690484 & 2.690484 & 6.284278\end{array}$

$\begin{array}{llll}6 & 1.807168 & 3.573443 & 5.365621\end{array}$

$\begin{array}{llll}6 & 2.689542 & 4.485861 & 4.485861\end{array}$

$\begin{array}{llll}6 & 1.807168 & 5.365621 & 3.573443\end{array}$

$\begin{array}{llll}6 & .895492 & 4.483739 & 2.691319\end{array}$

$\begin{array}{llll}6 & .013632 & 5.364275 & 1.779684\end{array}$

$\begin{array}{lllll}6 & -.897859 & 4.483095 & .897859\end{array}$

$\begin{array}{lllll}6 & -1.779684 & 5.364275 & -.013632\end{array}$

$\begin{array}{llll}1 & -2.417710 & 5.998738 & .624890\end{array}$

$\begin{array}{llll}6 & .896714 & 6.283029 & .896714\end{array}$

$\begin{array}{llll}6 & 1.793351 & 7.149529 & 1.793351\end{array}$

$\begin{array}{llll}6 & 2.690484 & 6.284278 & 2.690484\end{array}$

$\begin{array}{llll}6 & 3.587211 & 7.151577 & 3.587211\end{array}$

$\begin{array}{llll}6 & 4.485789 & 6.288559 & 4.485789\end{array}$

$\begin{array}{lllll}6 & 3.601434 & 5.369100 & 5.369100\end{array}$

$\begin{array}{lllll}6 & 4.485789 & 4.485789 & 6.288559\end{array}$

$\begin{array}{llll}6 & 5.369100 & 3.601434 & 5.369100\end{array}$

$\begin{array}{llll}6 & 4.485861 & 2.689542 & 4.485861\end{array}$

$\begin{array}{lllll}6 & 3.573443 & 1.807168 & 5.365621\end{array}$

$\begin{array}{lllll}1 & 4.210681 & 1.168087 & 6.000259\end{array}$

$\begin{array}{llll}6 & 3.586660 & 3.586660 & 3.586660\end{array}$

60 
\begin{tabular}{|llll}
\hline 6 & 5.369100 & 5.369100 & 3.601434
\end{tabular}

$\begin{array}{lllll}1 & 6.004194 & 6.004194 & 2.961112\end{array}$

$\begin{array}{lllll}6 & 7.159334 & 5.383621 & 5.383621\end{array}$

$\begin{array}{llll}6 & 6.274579 & 6.274579 & 6.274579\end{array}$

$\begin{array}{lllll}6 & 5.383621 & 5.383621 & 7.159334\end{array}$

$\begin{array}{llll}1 & 6.005813 & 4.758027 & 7.816194\end{array}$

$\begin{array}{lllll}1 & 4.758027 & 6.005813 & 7.816194\end{array}$

$\begin{array}{llll}6 & 5.383621 & 7.159334 & 5.383621\end{array}$

$\begin{array}{lllll}1 & 6.005813 & 7.816194 & 4.758027\end{array}$

$\begin{array}{llll}1 & 4.758027 & 7.816194 & 6.005813\end{array}$

$\begin{array}{lllll}1 & 6.908586 & 6.908586 & 6.908586\end{array}$

$\begin{array}{llll}1 & 7.816194 & 6.005813 & 4.758027\end{array}$

$\begin{array}{lllll}1 & 7.816194 & 4.758027 & 6.005813\end{array}$

$\begin{array}{llll}6 & 7.151577 & 3.587211 & 3.587211\end{array}$

$\begin{array}{lllll}1 & 7.810266 & 4.209917 & 2.962409\end{array}$

$\begin{array}{lllll}1 & 7.810266 & 2.962409 & 4.209917\end{array}$

$\begin{array}{lllll}1 & 6.004194 & 2.961112 & 6.004194\end{array}$

$\begin{array}{lllll}6 & 3.587211 & 3.587211 & 7.151577\end{array}$

$\begin{array}{lllll}1 & 2.962409 & 4.209917 & 7.810266\end{array}$

$\begin{array}{lllll}1 & 4.209917 & 2.962409 & 7.810266\end{array}$

$\begin{array}{llll}1 & 2.961112 & 6.004194 & 6.004194\end{array}$

$\begin{array}{llll}1 & 4.209917 & 7.810266 & 2.962409\end{array}$

$\begin{array}{llll}1 & 2.962409 & 7.810266 & 4.209917\end{array}$

$\begin{array}{lllll}1 & 2.416799 & 7.808066 & 1.169178\end{array}$

$\begin{array}{llll}1 & 1.169178 & 7.808066 & 2.416799\end{array}$

$\begin{array}{llll}6 & .000000 & 7.148945 & .000000\end{array}$

$\begin{array}{llll}1 & .623818 & 7.807467 & -.623818\end{array}$

$\begin{array}{lllll}1 & -.623818 & 7.807467 & .623818\end{array}$

$\begin{array}{llll}1 & -.624890 & 5.998738 & 2.417710\end{array}$

$\begin{array}{lllll}1 & 1.168087 & 6.000259 & 4.210681\end{array}$

$\begin{array}{llll}1 & 1.168087 & 4.210681 & 6.000259\end{array}$

$\begin{array}{lllll}1 & 1.169178 & 2.416799 & 7.808066\end{array}$

$\begin{array}{llll}1 & 2.416799 & 1.169178 & 7.808066\end{array}$

$\begin{array}{lllll}6 & .013632 & 1.779684 & 5.364275\end{array}$

$\begin{array}{llll}6 & -.897859 & .897859 & 4.483095\end{array}$

$\begin{array}{llll}6 & -1.778288 & 1.778288 & 3.570707\end{array}$

$\begin{array}{lllll}1 & -2.415747 & 2.415747 & 4.207200\end{array}$

$\begin{array}{lllll}1 & -.624890 & 2.417710 & 5.998738\end{array}$

$\begin{array}{llll}6 & .000000 & .000000 & 7.148945\end{array}$

$\begin{array}{lllll}1 & -.623818 & .623818 & 7.807467\end{array}$

$\begin{array}{llll}1 & .623818 & -.623818 & 7.807467\end{array}$

$\begin{array}{lllll}1 & 2.417710 & -.624890 & 5.998738\end{array}$

$\begin{array}{lllll}1 & 4.207982 & -.623617 & 4.207982\end{array}$

$\begin{array}{llll}6 & 5.364275 & .013632 & 1.779684\end{array}$

$\begin{array}{llll}6 & 6.283029 & .896714 & .896714\end{array}$

$\begin{array}{lllll}6 & 5.364275 & 1.779684 & .013632\end{array}$

$\begin{array}{lllll}6 & 4.483739 & 2.691319 & .895492\end{array}$

$\begin{array}{llll}6 & 3.571353 & 3.571353 & .014493\end{array}$

$\begin{array}{lllll}1 & 4.207982 & 4.207982 & -.623617\end{array}$

$\begin{array}{llll}6 & 3.584108 & 1.793125 & 1.793125\end{array}$

$\begin{array}{lllll}6 & 4.483095 & .897859 & -.897859\end{array}$

$\begin{array}{lllll}6 & 3.583331 & .000000 & .000000\end{array}$

$\begin{array}{llll}1 & 5.998738 & 2.417710 & -.624890 \\ 6 & 7.149529 & 1.793351 & 1.793351\end{array}$

$\begin{array}{llll}6 & 7.149529 & 1.793351 & 1.793351\end{array}$

$\begin{array}{lllll}1 & 7.808066 & 2.416799 & 1.169178\end{array}$

$\begin{array}{llll}1 & 7.808066 & 1.169178 & 2.416799\end{array}$

$\begin{array}{lllll}6 & 7.148945 & .000000 & .000000\end{array}$

$\begin{array}{llll}1 & 7.807467 & .623818 & -.623818\end{array}$

$\begin{array}{lllll}1 & 7.807467 & -.623818 & .623818\end{array}$

$\begin{array}{llll}1 & 5.998738 & -.624890 & 2.417710\end{array}$

$\begin{array}{llll}1 & 6.000259 & 1.168087 & 4.210681\end{array}$

$\begin{array}{llll}1 & 6.000259 & 4.210681 & 1.168087\end{array}$

$\begin{array}{lllll}1 & 4.210681 & 6.000259 & 1.168087\end{array}$

$\begin{array}{lllll}1 & 2.417710 & 5.998738 & -.624890\end{array}$

$\begin{array}{lllll}6 & 1.778288 & 3.570707 & -1.778288\end{array}$

$\begin{array}{lllll}1 & 2.415747 & 4.207200 & -2.415747\end{array}$

$\begin{array}{lllll}1 & .624890 & 5.998738 & -2.417710\end{array}$

$\begin{array}{lllll}1 & -1.169178 & 7.808066 & -2.416799\end{array}$

$\begin{array}{lllll}1 & -2.416799 & 7.808066 & -1.169178\end{array}$

$\begin{array}{llll}6 & -3.587211 & 7.151577 & -3.587211\end{array}$

\begin{tabular}{lllll}
6 & -4.485789 & 6.288559 & -4.485789 \\
\hline
\end{tabular} 
\begin{tabular}{llll}
\hline 6 & -3.601434 & 5.369100 & -5.369100
\end{tabular}

$\begin{array}{lllll}6 & -4.485789 & 4.485789 & -6.288559\end{array}$

$\begin{array}{lllll}6 & -3.587211 & 3.587211 & -7.151577\end{array}$

$\begin{array}{llll}6 & -2.690484 & 2.690484 & -6.284278\end{array}$

$\begin{array}{lllll}6 & -1.793351 & 1.793351 & -7.149529\end{array}$

$\begin{array}{llll}1 & -2.416799 & 1.169178 & -7.808066\end{array}$

$\begin{array}{lllll}1 & -1.169178 & 2.416799 & -7.808066\end{array}$

$\begin{array}{llll}6 & -3.573443 & 1.807168 & -5.365621\end{array}$

$\begin{array}{lllll}1 & -4.210681 & 1.168087 & -6.000259\end{array}$

$\begin{array}{llll}1 & -4.209917 & 2.962409 & -7.810266\end{array}$

$\begin{array}{lllll}1 & -2.962409 & 4.209917 & -7.810266\end{array}$

$\begin{array}{llll}6 & -5.383621 & 5.383621 & -7.159334\end{array}$

$\begin{array}{lllll}1 & -6.005813 & 4.758027 & -7.816194\end{array}$

$\begin{array}{llll}1 & -4.758027 & 6.005813 & -7.816194\end{array}$

$\begin{array}{lllll}6 & -6.274579 & 6.274579 & -6.274579\end{array}$

$\begin{array}{lllll}6 & -7.159334 & 5.383621 & -5.383621\end{array}$

$\begin{array}{lllll}1 & -7.816194 & 6.005813 & -4.758027\end{array}$

$\begin{array}{lllll}1 & -7.816194 & 4.758027 & -6.005813\end{array}$

$\begin{array}{lllll}1 & -6.908586 & 6.908586 & -6.908586\end{array}$

$\begin{array}{lllll}6 & -5.383621 & 7.159334 & -5.383621\end{array}$

$\begin{array}{lllll}1 & -6.005813 & 7.816194 & -4.758027\end{array}$

$\begin{array}{lllll}1 & -4.758027 & 7.816194 & -6.005813\end{array}$

$\begin{array}{lllll}1 & -2.961112 & 6.004194 & -6.004194\end{array}$

$\begin{array}{lllll}6 & -5.369100 & 5.369100 & -3.601434\end{array}$

$\begin{array}{lllll}1 & -6.004194 & 6.004194 & -2.961112\end{array}$

$\begin{array}{llll}1 & -2.962409 & 7.810266 & -4.209917\end{array}$

$\begin{array}{llll}1 & -4.209917 & 7.810266 & -2.962409\end{array}$

$\begin{array}{lllll}1 & -1.168087 & 6.000259 & -4.210681\end{array}$

$\begin{array}{lllll}1 & -1.168087 & 4.210681 & -6.000259\end{array}$

$\begin{array}{lllll}1 & .624890 & 2.417710 & -5.998738\end{array}$

$\begin{array}{llll}6 & .000000 & .000000 & -7.148945\end{array}$

$\begin{array}{lllll}1 & -.623818 & -.623818 & -7.807467\end{array}$

$\begin{array}{lllll}1 & .623818 & .623818 & -7.807467\end{array}$

$\begin{array}{lllll}1 & -2.417710 & -.624890 & -5.998738\end{array}$

$\begin{array}{llll}1 & -4.207982 & -.623617 & -4.207982\end{array}$

$\begin{array}{lllll}6 & -2.690143 & -2.690143 & -.897329\end{array}$

$\begin{array}{lllll}6 & -1.791447 & -1.791447 & .000933\end{array}$

$\begin{array}{lllll}1 & -4.207200 & -2.415747 & -2.415747\end{array}$

$\begin{array}{llll}6 & -3.583331 & .000000 & .000000\end{array}$

$\begin{array}{lllll}1 & -5.998738 & -2.417710 & -.624890\end{array}$

$\begin{array}{llll}6 & -7.149529 & -1.793351 & 1.793351\end{array}$

$\begin{array}{lllll}1 & -7.808066 & -2.416799 & 1.169178\end{array}$

$\begin{array}{lllll}1 & -7.808066 & -1.169178 & 2.416799\end{array}$

$\begin{array}{lllll}1 & -7.807467 & -.623818 & -.623818\end{array}$

$\begin{array}{lllll}1 & -7.807467 & .623818 & .623818\end{array}$

$\begin{array}{lllll}6 & -7.149529 & 1.793351 & -1.793351\end{array}$

$\begin{array}{llll}1 & -7.808066 & 1.169178 & -2.416799\end{array}$

$\begin{array}{lllll}1 & -7.808066 & 2.416799 & -1.169178\end{array}$

$\begin{array}{lllll}1 & -5.998738 & -.624890 & -2.417710\end{array}$

$\begin{array}{lllll}1 & -6.000259 & 1.168087 & -4.210681\end{array}$

$\begin{array}{llll}6 & -5.365621 & 3.573443 & -1.807168\end{array}$

$\begin{array}{lllll}1 & -6.000259 & 4.210681 & -1.168087\end{array}$

$\begin{array}{lllll}1 & -7.810266 & 2.962409 & -4.209917\end{array}$

$\begin{array}{llll}1 & -7.810266 & 4.209917 & -2.962409\end{array}$

$\begin{array}{lllll}1 & -6.004194 & 2.961112 & -6.004194\end{array}$

$\begin{array}{lllll}1 & -4.210681 & 6.000259 & -1.168087\end{array}$

$\begin{array}{lllll}6 & -3.571353 & 3.571353 & -.014493\end{array}$

$\begin{array}{llll}1 & -4.207982 & 4.207982 & .623617\end{array}$

$\begin{array}{lllll}1 & .623617 & 4.207982 & -4.207982\end{array}$

$\begin{array}{llll}1 & 2.415747 & 2.415747 & -4.207200\end{array}$

$\begin{array}{lllll}1 & -5.998738 & 2.417710 & .624890\end{array}$

$\begin{array}{llll}6 & -3.570707 & 1.778288 & 1.778288\end{array}$

$\begin{array}{lllll}1 & -4.207200 & 2.415747 & 2.415747\end{array}$

$\begin{array}{lllll}1 & -5.998738 & .624890 & 2.417710\end{array}$

$\begin{array}{lllll}1 & -4.207982 & .623617 & 4.207982\end{array}$

$\begin{array}{lllll}1 & -2.417710 & .624890 & 5.998738\end{array}$

$\begin{array}{lllll}1 & -1.169178 & -2.416799 & 7.808066\end{array}$

$\begin{array}{llll}1 & -2.416799 & -1.169178 & 7.808066\end{array}$

$\begin{array}{llll}1 & -4.210681 & -1.168087 & 6.000259\end{array}$

$\begin{array}{llll}1 & -6.000259 & -1.168087 & 4.210681\end{array}$

\begin{tabular}{llll}
1 & -7.810266 & -4.209917 & 2.962409 \\
\hline
\end{tabular} 
$\begin{array}{lllll}1 & -7.810266 & -2.962409 & 4.209917\end{array}$

$\begin{array}{llll}1 & -7.816194 & -6.005813 & 4.758027\end{array}$

$\begin{array}{lllll}1 & -7.816194 & -4.758027 & 6.005813\end{array}$

$\begin{array}{llll}1 & -6.908586 & -6.908586 & 6.908586\end{array}$

$\begin{array}{lllll}1 & -6.005813 & -7.816194 & 4.758027\end{array}$

$\begin{array}{llll}1 & -4.758027 & -7.816194 & 6.005813\end{array}$

$\begin{array}{lllll}1 & -6.004194 & -6.004194 & 2.961112\end{array}$

$\begin{array}{llll}1 & -6.000259 & -4.210681 & 1.168087\end{array}$

$\begin{array}{lllll}1 & -4.207982 & -4.207982 & -.623617\end{array}$

$\begin{array}{llll}1 & -2.417710 & -5.998738 & -.624890\end{array}$

$\begin{array}{lllll}1 & -2.415747 & -4.207200 & -2.415747\end{array}$

$\begin{array}{lllll}1 & -2.415747 & -2.415747 & -4.207200\end{array}$

$\begin{array}{lllll}1 & -.624890 & -2.417710 & -5.998738\end{array}$

$\begin{array}{lllll}6 & 1.807168 & -3.573443 & -5.365621\end{array}$

$\begin{array}{llll}1.168087 & -4.210681 & -6.000259\end{array}$

$\begin{array}{lllll}1 & -.623617 & -4.207982 & -4.207982\end{array}$

$\begin{array}{llll}1.168087 & -6.000259 & -4.210681\end{array}$

$\begin{array}{llll}2.961112 & -6.004194 & -6.004194\end{array}$

$\begin{array}{llll}4.485789 & -4.485789 & -6.288559\end{array}$

$\begin{array}{llll}5.369100 & -3.601434 & -5.369100\end{array}$

$\begin{array}{llll}6.004194 & -2.961112 & -6.004194\end{array}$

$\begin{array}{lllll}6 & 5.383621 & -5.383621 & -7.159334\end{array}$

$\begin{array}{lllll}1 & 4.758027 & -6.005813 & -7.816194\end{array}$

$\begin{array}{lllll}6 & 6.274579 & -6.274579 & -6.274579\end{array}$

$6 \begin{array}{llll}6 & 7.159334 & -5.383621 & -5.383621\end{array}$

$\begin{array}{lllll}1 & 7.816194 & -6.005813 & -4.758027\end{array}$

$\begin{array}{llll}1 & 7.816194 & -4.758027 & -6.005813\end{array}$

$\begin{array}{lllll}1 & 6.908586 & -6.908586 & -6.908586\end{array}$

$\begin{array}{llll}6 & 5.383621 & -7.159334 & -5.383621\end{array}$

$\begin{array}{lllll}1 & 6.005813 & -7.816194 & -4.758027\end{array}$

$\begin{array}{llll}1 & 4.758027 & -7.816194 & -6.005813\end{array}$

$1 \quad 6.005813 \quad-4.758027 \quad-7.816194$

$\begin{array}{llll}6 & 3.587211 & -3.587211 & -7.151577\end{array}$

$\begin{array}{lllll}1 & 2.962409 & -4.209917 & -7.810266\end{array}$

$\begin{array}{llll}1 & 4.209917 & -2.962409 & -7.810266\end{array}$

$\begin{array}{lllll}1 & 6.000259 & -4.210681 & -1.168087\end{array}$

$\begin{array}{lllll}1 & 4.210681 & -1.168087 & -6.000259\end{array}$

$\begin{array}{lllll}1 & 4.207982 & .623617 & -4.207982\end{array}$

$\begin{array}{lllll}1 & 4.207200 & 2.415747 & -2.415747\end{array}$

$\begin{array}{lllll}1 & 4.207200 & -2.415747 & 2.415747\end{array}$

$\begin{array}{llll}1 & 5.998738 & -2.417710 & .624890\end{array}$

$\begin{array}{lllll}1 & 5.998738 & .624890 & -2.417710\end{array}$

$\begin{array}{llll}1 & 4.207982 & -4.207982 & .623617\end{array}$

$\begin{array}{lllll}1 & .623617 & -4.207982 & 4.207982\end{array}$

$\begin{array}{llll}1 & 2.415747 & -4.207200 & 2.415747\end{array}$

$\begin{array}{lllll}1 & -.623617 & 4.207982 & 4.207982\end{array}$

$\begin{array}{llll}1 & -2.415747 & 4.207200 & 2.415747\end{array}$

$\mathrm{C}_{16} \mathrm{H}_{21} \mathrm{~F}_{3}$ RB3PW91\6-31G(d,p) E=-921.649850

$\mathrm{C}_{16} \mathrm{H}_{24}$ RB3PW91\6-31G(d,p) E=-624.03403

$\begin{array}{llll}\mathrm{H} & 2.149454 & -1.240988 & 0.814014\end{array}$

$\begin{array}{llll}\mathrm{H} & 0.000000 & 2.481976 & 0.814014\end{array}$

$\begin{array}{llll}\mathrm{H} & -2.149454 & -1.240988 & 0.814014\end{array}$

$\begin{array}{llll}\mathrm{H} & 2.153935 & 0.225381 & 2.905335\end{array}$

H $1.272153 \quad 1.752672 \quad 2.905335$

$\mathrm{H} \quad-1.272153 \quad 1.752672 \quad 2.905335$

H $\quad 0.881782-1.978053 \quad 2.905335$

$\mathrm{H} \quad-0.881782-1.978053 \quad 2.905335$

$\begin{array}{llll}\mathrm{H} & -2.153935 & 0.225381 & 2.905335\end{array}$

C $\quad 1.254182 \quad-0.724102 \quad-1.077510$

C $\quad 0.000000 \quad 1.448204 \quad-1.077510$

$\begin{array}{llll}\text { C } & -1.254182 & -0.724102 & -1.077510\end{array}$

$\begin{array}{llll}\text { C } & 0.000000 & 0.000000 & -1.602543\end{array}$

$\begin{array}{llll}\text { C } & 0.000000 & 1.443151 & 0.454782\end{array}$

$\begin{array}{llll}\text { C } & 1.249806 & -0.721576 & 0.454782\end{array}$

$\begin{array}{llll}\text { C } & -1.249806 & -0.721576 & 0.454782\end{array}$

H $\quad 2.154829 \quad-0.224990 \quad-1.454461$

$\begin{array}{llll}\mathrm{H} & 0.882567 & 1.978632 & -1.454461\end{array}$

$\mathrm{H} \quad-0.882567 \quad 1.978632 \quad-1.454461$

$\begin{array}{llll}\mathrm{H} & 1.272262 & -1.753641 & -1.454461\end{array}$

$\begin{array}{llll}\mathrm{H} & -1.272262 & -1.753641 & -1.454461\end{array}$
$\begin{array}{llll}\mathrm{H} & 0.000000 & 2.481770 & 0.091848\end{array}$

$\begin{array}{llll}\mathrm{H} & -2.149276 & -1.240885 & 0.091848\end{array}$

$\begin{array}{llll}\mathrm{H} & 2.149276 & 1.240885 & -0.091848\end{array}$

$\begin{array}{lllll}\mathrm{H} & 0.000000 & -2.481770 & -0.091848\end{array}$

$\begin{array}{llll}\mathrm{H} & 2.149276 & -1.240885 & 0.091848\end{array}$

$\begin{array}{llll}\mathrm{H} & -2.149276 & 1.240885 & -0.091848\end{array}$

$\begin{array}{llll}\mathrm{H} & -1.271452 & 1.752638 & 2.180685\end{array}$

$\begin{array}{llll}\mathrm{H} & -2.153555 & 0.224791 & 2.180685\end{array}$

$\begin{array}{llll}\mathrm{H} & 2.153555 & -0.224791 & -2.180685\end{array}$

$\begin{array}{llll}\mathrm{H} & 1.271452 & -1.752638 & -2.180685\end{array}$

$\begin{array}{llll}\mathrm{H} & -0.882103 & -1.977429 & 2.180685\end{array}$

$\begin{array}{llll}\mathrm{H} & 1.271452 & 1.752638 & 2.180685\end{array}$

$\begin{array}{llll}\mathrm{H} & -1.271452 & -1.752638 & -2.180685\end{array}$

$\begin{array}{llll}\mathrm{H} & 0.882103 & 1.977429 & -2.180685\end{array}$

$\begin{array}{llll}\mathrm{H} & 2.153555 & 0.224791 & 2.180685\end{array}$

$\begin{array}{llll}\mathrm{H} & 0.882103 & -1.977429 & 2.180685\end{array}$

$\begin{array}{llll}\mathrm{H} & -0.882103 & 1.977429 & -2.180685\end{array}$

$\begin{array}{llll}\mathrm{H} & -2.153555 & -0.224791 & -2.180685\end{array}$

$\begin{array}{lllll}\text { C } & 0.000000 & 1.440266 & -1.804043\end{array}$

$\begin{array}{llll}\text { C } & -1.247307 & -0.720133 & -1.804043\end{array}$ $\begin{array}{llll}\text { C } & 1.247307 & 0.720133 & 1.804043\end{array}$ 
2

3

4

5

6

7

8

9

10

11

12

13

14

15

16

17

18

19

20

21

22

23

24

25

26

27

28

29

30

31

32

33

34

35

36

37

38

39

40

41

42

43

44

45

46

47

48

49

50

51

52

53

54

55

56

57

58

59

60

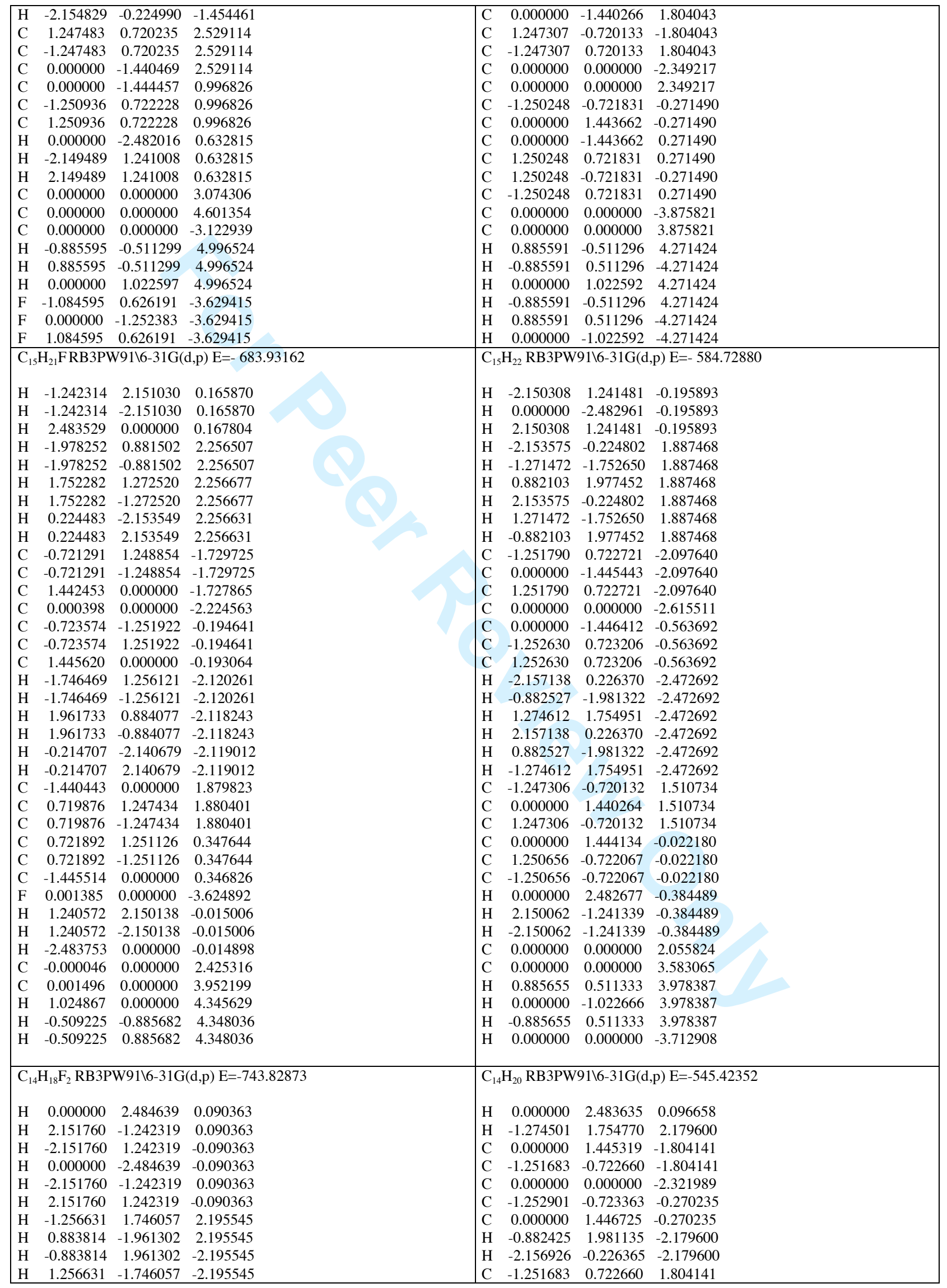

URL: http://mc.manuscriptcentral.com/tandf/tmph 
2

3

4

5

6

7

8

9

10

11

12

13

14

15

16

17

18

19

20

21

22

23

24

25

26

27

28

29

30

31

32

33

34

35

36

37

38

39

40

41

42

43

44

45

46

47

48

49

50

51

52

53

54

55

56

57

58

59

60

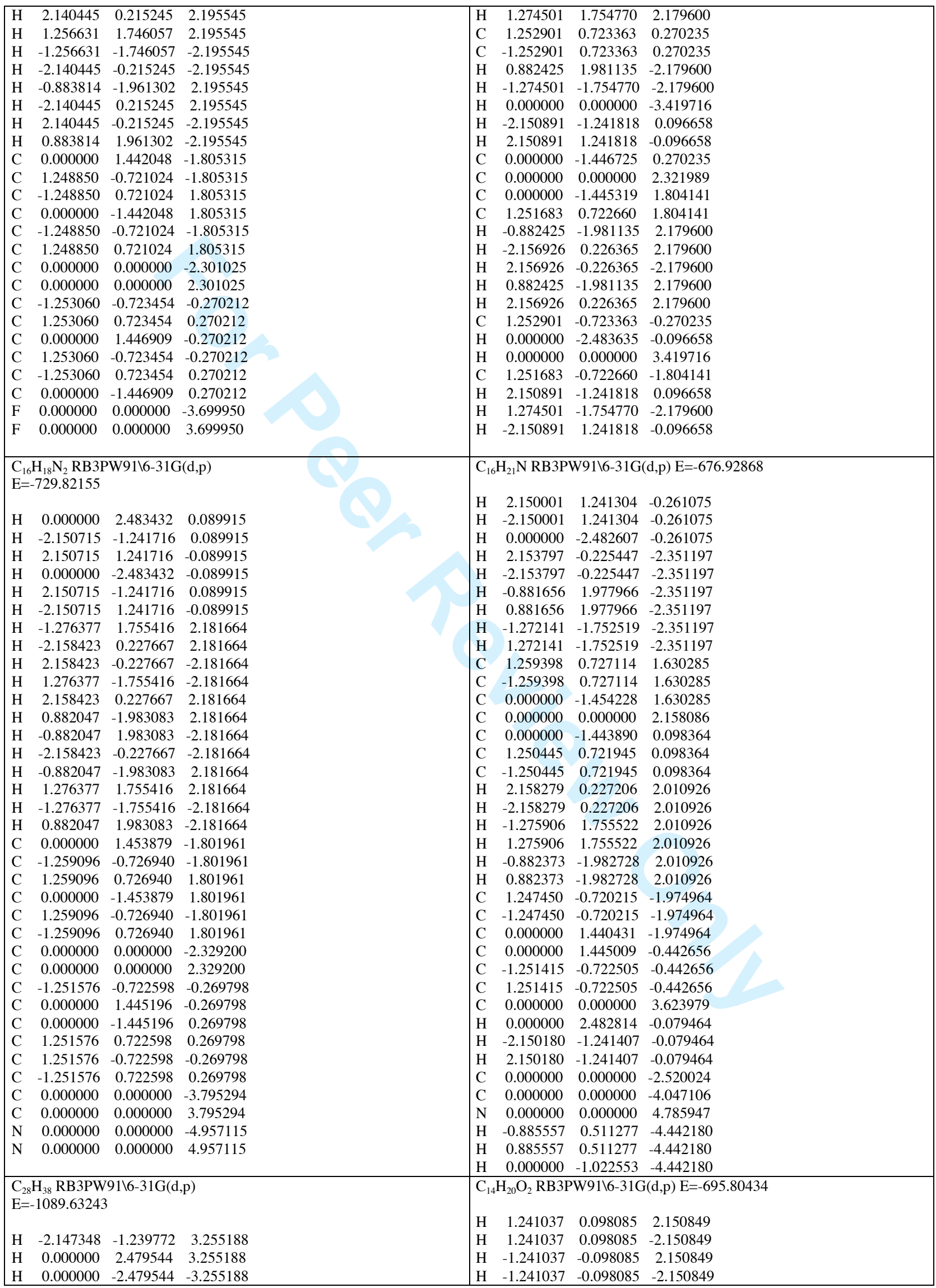

URL: http://mc.manuscriptcentral.com/tandf/tmph 
2

3

4

5

6

7

8

9

10

11

12

13

14

15

16

17

18

19

20

21

22

23

24

25

26

27

28

29

30

31

32

33

34

35

36

37

38

39

40

41

42

43

44

45

46

47

48

49

50

51

52

53

54

55

56

57

58

59

60

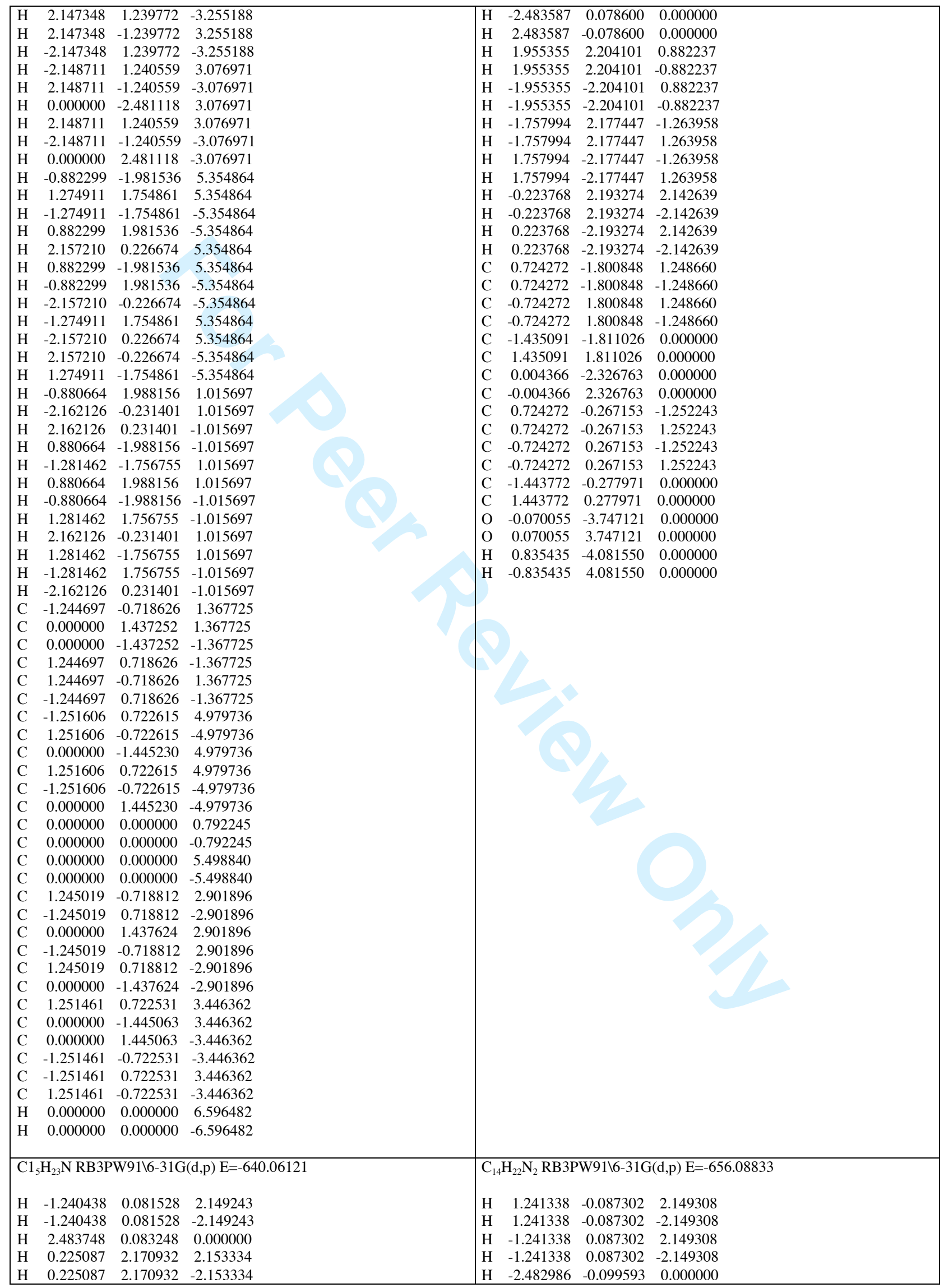

URL: http://mc.manuscriptcentral.com/tandf/tmph 
2

3

4

5

6

7

8

9

10

11

12

13

14

15

16

17

18

19

20

21

22

23

24

25

26

27

28

29

30

31

32

33

34

35

36

37

38

39

40

41

42

43

44

45

46

47

48

49

50

51

52

53

54

55

56

57

58

59

60

\begin{tabular}{|c|c|c|c|c|c|c|c|}
\hline $\mathrm{H}$ & -1.976166 & 2.172104 & -0.881696 & $\mathrm{H}$ & 2.482986 & 0.099593 & 0.000000 \\
\hline $\mathrm{H}$ & -1.976166 & 2.172104 & 0.881696 & $\mathrm{H}$ & -0.221229 & -2.184567 & 2.149731 \\
\hline $\mathrm{H}$ & 1.753647 & 2.171231 & -1.272721 & $\mathrm{H}$ & -0.221229 & -2.184567 & -2.149731 \\
\hline $\mathrm{H}$ & 1.753647 & 2.171231 & 1.272721 & $\mathrm{H}$ & 0.221229 & 2.184567 & 2.149731 \\
\hline $\mathrm{C}$ & -0.726684 & -1.813667 & 1.245881 & $\mathrm{H}$ & 0.221229 & 2.184567 & -2.149731 \\
\hline $\mathrm{C}$ & -0.726684 & -1.813667 & -1.245881 & $\mathrm{H}$ & 1.974269 & -2.179361 & -0.883118 \\
\hline $\mathrm{C}$ & 1.435482 & -1.812105 & 0.000000 & $\mathrm{H}$ & 1.974269 & -2.179361 & 0.883118 \\
\hline $\mathrm{C}$ & -0.009070 & -2.352841 & 0.000000 & $\mathrm{H}$ & -1.974269 & 2.179361 & -0.883118 \\
\hline $\mathrm{C}$ & 1.445341 & -0.279153 & 0.000000 & $\mathrm{H}$ & -1.974269 & 2.179361 & 0.883118 \\
\hline $\mathrm{C}$ & -0.721851 & -0.281293 & 1.250057 & $\mathrm{H}$ & -1.746441 & -2.197539 & -1.260546 \\
\hline $\mathrm{C}$ & -0.721851 & -0.281293 & -1.250057 & $\mathrm{H}$ & -1.746441 & -2.197539 & 1.260546 \\
\hline $\mathrm{H}$ & -0.227764 & -2.193429 & 2.149800 & $\mathrm{H}$ & 1.746441 & 2.197539 & -1.260546 \\
\hline $\mathrm{H}$ & -0.227764 & -2.193429 & -2.149800 & $\mathrm{H}$ & 1.746441 & 2.197539 & 1.260546 \\
\hline $\mathrm{H}$ & -1.752877 & -2.201254 & -1.260017 & $\mathrm{C}$ & 0.721629 & 1.806404 & 1.246091 \\
\hline $\mathrm{H}$ & -1.752877 & -2.201254 & 1.260017 & $\mathrm{C}$ & 0.721629 & 1.806404 & -1.246091 \\
\hline $\mathrm{H}$ & 1.968164 & -2.194305 & -0.883216 & $\mathrm{C}$ & -0.721629 & -1.806404 & 1.246091 \\
\hline $\mathrm{H}$ & 1.968164 & -2.194305 & 0.883216 & $\mathrm{C}$ & -0.721629 & -1.806404 & -1.246091 \\
\hline $\mathrm{C}$ & 0.721178 & 1.794639 & 1.247448 & $\mathrm{C}$ & -1.440104 & 1.799073 & 0.000000 \\
\hline $\mathrm{C}$ & 0.721178 & 1.794639 & -1.247448 & $\mathrm{C}$ & 1.440104 & -1.799073 & 0.000000 \\
\hline $\mathrm{C}$ & -1.438638 & 1.795003 & 0.000000 & $\mathrm{C}$ & 0.002505 & 2.343240 & 0.000000 \\
\hline $\mathrm{C}$ & -1.443555 & 0.262399 & 0.000000 & $\mathrm{C}$ & -0.002505 & -2.343240 & 0.000000 \\
\hline $\mathrm{C}$ & 0.722492 & 0.262003 & -1.250326 & $\mathrm{C}$ & -1.446112 & 0.265859 & 0.000000 \\
\hline $\mathrm{C}$ & 0.722492 & 0.262003 & 1.250326 & $\mathrm{C}$ & 1.446112 & -0.265859 & 0.000000 \\
\hline $\mathrm{N}$ & -0.099211 & -3.812754 & 0.000000 & $\mathrm{C}$ & 0.721629 & 0.273965 & 1.250189 \\
\hline $\mathrm{H}$ & -2.481624 & -0.099884 & 0.000000 & $\mathrm{C}$ & 0.721629 & 0.273965 & -1.250189 \\
\hline $\mathrm{H}$ & 1.241399 & -0.101028 & -2.149599 & $\mathrm{C}$ & -0.721629 & -0.273965 & 1.250189 \\
\hline $\mathrm{H}$ & 1.241399 & -0.101028 & 2.149599 & $\mathrm{C}$ & -0.721629 & -0.273965 & -1.250189 \\
\hline $\mathrm{C}$ & 0.001692 & 2.340272 & 0.000000 & $\mathrm{~N}$ & 0.089308 & 3.803773 & 0.000000 \\
\hline $\mathrm{C}$ & 0.003698 & 3.867317 & 0.000000 & $\mathrm{~N}$ & -0.089308 & -3.803773 & 0.000000 \\
\hline $\mathrm{H}$ & -0.507075 & 4.263342 & -0.885591 & $\mathrm{H}$ & -0.404829 & 4.168714 & -0.812295 \\
\hline $\mathrm{H}$ & -0.507075 & 4.263342 & 0.885591 & $\mathrm{H}$ & -0.404829 & 4.168714 & 0.812295 \\
\hline $\mathrm{H}$ & 1.027016 & 4.260941 & 0.000000 & $\mathrm{H}$ & 0.404829 & -4.168714 & -0.812295 \\
\hline $\mathrm{H}$ & 0.393034 & -4.179396 & -0.812501 & $\mathrm{H}$ & 0.404829 & -4.168714 & 0.812295 \\
\hline $\mathrm{H}$ & 0.393034 & -4.179396 & 0.812501 & & & & \\
\hline \multicolumn{4}{|c|}{$\mathrm{C}_{16} \mathrm{H}_{21} \mathrm{~F}$ RB3PW91\6-31G(d,p) E=-721.98753 } & \multicolumn{4}{|c|}{$\mathrm{C}_{14} \mathrm{H}_{22} \mathrm{~N}(1+) \mathrm{RB} 3 \mathrm{PW} 91 \backslash 6-31 \mathrm{G}(\mathrm{d}, \mathrm{p}) \mathrm{E}=-601.14291$} \\
\hline $\mathrm{H}$ & 1.290962 & 0.258032 & 2.151206 & $\mathrm{H}$ & -2.152684 & -1.242853 & -0.396011 \\
\hline $\mathrm{H}$ & 1.290962 & 0.258032 & -2.151206 & $\mathrm{H}$ & 2.152684 & -1.242853 & -0.396011 \\
\hline $\mathrm{H}$ & -2.425250 & -0.002638 & 0.000000 & $\mathrm{H}$ & 0.000000 & 2.485706 & -0.396011 \\
\hline $\mathrm{H}$ & -0.025935 & -1.931037 & 2.155121 & $\mathrm{H}$ & -2.158710 & 0.229945 & -2.491104 \\
\hline $\mathrm{H}$ & -0.025935 & -1.931037 & -2.155121 & $\mathrm{H}$ & 2.158710 & 0.229945 & -2.491104 \\
\hline $\mathrm{H}$ & 2.171352 & -1.773649 & -0.881803 & $\mathrm{H}$ & 1.278493 & 1.754525 & -2.491104 \\
\hline $\mathrm{H}$ & 2.171352 & -1.773649 & 0.881803 & $\mathrm{H}$ & -1.278493 & 1.754525 & -2.491104 \\
\hline $\mathrm{H}$ & -1.547965 & -2.036978 & -1.277551 & $\mathrm{H}$ & 0.880217 & -1.984470 & -2.491104 \\
\hline $\mathrm{H}$ & -1.547965 & -2.036978 & 1.277551 & $\mathrm{H}$ & -0.880217 & -1.984470 & -2.491104 \\
\hline $\mathrm{C}$ & 0.638113 & 2.112829 & 1.248864 & $\mathrm{C}$ & -1.256946 & -0.725698 & 1.488564 \\
\hline $\mathrm{C}$ & 0.638113 & 2.112829 & -1.248864 & $\mathrm{C}$ & 1.256946 & -0.725698 & 1.488564 \\
\hline $\mathrm{C}$ & -1.519271 & 1.961552 & 0.000000 & $\mathrm{C}$ & 0.000000 & 1.451396 & 1.488564 \\
\hline $\mathrm{C}$ & -0.115720 & 2.556596 & 0.000000 & $\mathrm{C}$ & 0.000000 & 0.000000 & 1.966016 \\
\hline $\mathrm{C}$ & -1.415048 & 0.430115 & 0.000000 & $\mathrm{C}$ & 0.000000 & 1.445203 & -0.049430 \\
\hline $\mathrm{C}$ & 0.748435 & 0.582117 & 1.252238 & $\mathrm{C}$ & 1.251583 & -0.722602 & -0.049430 \\
\hline $\mathrm{C}$ & 0.748435 & 0.582117 & -1.252238 & $\mathrm{C}$ & -1.251583 & -0.722602 & -0.049430 \\
\hline $\mathrm{H}$ & 0.104916 & 2.466445 & 2.140134 & $\mathrm{H}$ & -2.158072 & -0.220436 & 1.863423 \\
\hline $\mathrm{H}$ & 0.104916 & 2.466445 & -2.140134 & $\mathrm{H}$ & 2.158072 & -0.220436 & 1.863423 \\
\hline $\mathrm{H}$ & 1.632433 & 2.576358 & -1.257134 & $\mathrm{H}$ & 0.888133 & 1.979163 & 1.863423 \\
\hline $\mathrm{H}$ & 1.632433 & 2.576358 & 1.257134 & $\mathrm{H}$ & -0.888133 & 1.979163 & 1.863423 \\
\hline $\mathrm{H}$ & -2.064400 & 2.314880 & -0.884056 & $\mathrm{H}$ & 1.269939 & -1.758727 & 1.863423 \\
\hline $\mathrm{H}$ & -2.064400 & 2.314880 & 0.884056 & $\mathrm{H}$ & -1.269939 & -1.758727 & 1.863423 \\
\hline $\mathrm{C}$ & -0.546879 & -1.588287 & 1.251392 & $\mathrm{C}$ & -1.252301 & 0.723016 & -2.118976 \\
\hline $\mathrm{C}$ & -0.546879 & -1.588287 & -1.251392 & $\mathrm{C}$ & 1.252301 & 0.723016 & -2.118976 \\
\hline $\mathrm{C}$ & 1.609405 & -1.435428 & 0.000000 & $\mathrm{C}$ & 0.000000 & -1.446033 & -2.118976 \\
\hline $\mathrm{C}$ & 1.505967 & 0.092413 & 0.000000 & $\mathrm{C}$ & 0.000000 & -1.450653 & -0.584880 \\
\hline $\mathrm{C}$ & -0.655150 & -0.059470 & -1.250732 & $\mathrm{C}$ & -1.256303 & 0.725327 & -0.584880 \\
\hline $\mathrm{C}$ & -0.655150 & -0.059470 & 1.250732 & $\mathrm{C}$ & 1.256303 & 0.725327 & -0.584880 \\
\hline $\mathrm{H}$ & 2.516584 & 0.525329 & 0.000000 & $\mathrm{~N}$ & 0.000000 & 0.000000 & 3.502570 \\
\hline $\mathrm{H}$ & -1.197829 & 0.265429 & -2.150085 & $\mathrm{H}$ & 0.000000 & -2.487762 & -0.220237 \\
\hline $\mathrm{H}$ & -1.197829 & 0.265429 & 2.150085 & $\mathrm{H}$ & -2.154465 & 1.243881 & -0.220237 \\
\hline $\mathrm{C}$ & 0.211671 & -2.084672 & 0.000000 & $\mathrm{H}$ & 2.154465 & 1.243881 & -0.220237 \\
\hline $\mathrm{C}$ & 0.341354 & -3.595492 & 0.000000 & $\mathrm{C}$ & 0.000000 & 0.000000 & -2.634888 \\
\hline $\mathrm{C}$ & -0.665391 & -4.469149 & 0.000000 & $\mathrm{H}$ & 0.828818 & 0.478518 & 3.868497 \\
\hline $\mathrm{F}$ & -0.213264 & 3.953187 & 0.000000 & $\mathrm{H}$ & -0.828818 & 0.478518 & 3.868497 \\
\hline
\end{tabular}


2

3

4

5

6

7

8

9

10

11

12

13

14

15

16

17

18

19

20

21

22

23

24

25

26

27

28

29

30

31

32

33

34

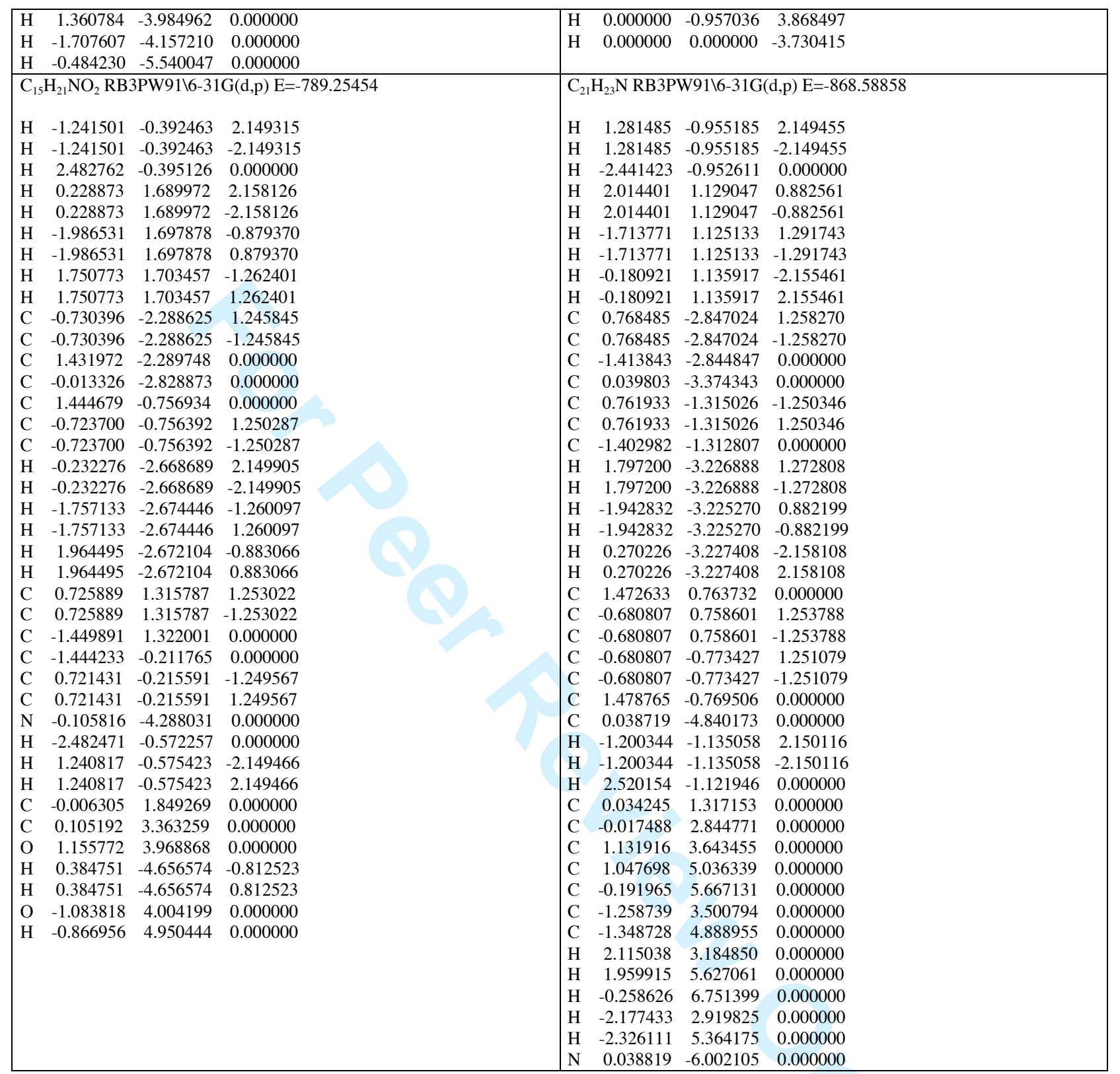

URL: http://mc.manuscriptcentral.com/tandf/tmph 
2

3

4

5

6

7

8

9

10

11

12

13

14

15

16

17

18

19

20

21

22

23

24

25

26

27

28

29

30

31

32

33

34

35

36

37

38

39

40

41

42

43

44

45

46

47

48

49

50

51

52

53

54

55

56

57

58

59

60

\begin{tabular}{|c|c|c|c|c|c|}
\hline \multicolumn{3}{|c|}{$\mathrm{C}_{20} \mathrm{H}_{23}$ F RB3PW91\6-31G(d,p) E=-875.59164 } & \multicolumn{3}{|c|}{$\mathrm{C}_{14} \mathrm{H}_{18} \mathrm{~N}_{2} \mathrm{O}_{4}$ RB3PW9116-31G(d,p) E=-954.27746 } \\
\hline $\mathrm{H}$ & 1.284871 & $-1.140510 \quad 2.150633$ & $\mathrm{H} \quad-1.243044$ & 0.083403 & 2.150980 \\
\hline $\mathrm{H}$ & 1.284871 & $-1.140510-2.150633$ & H $\quad-1.243044$ & 0.083403 & -2.150980 \\
\hline $\mathrm{H}$ & -2.439987 & $-1.139361 \quad 0.000000$ & $\mathrm{H} \quad 1.243044$ & -0.083403 & 2.150980 \\
\hline $\mathrm{H}$ & 2.015274 & $0.944406 \quad 0.882592$ & $\mathrm{H} \quad 1.243044$ & -0.083403 & -2.150980 \\
\hline $\mathrm{H}$ & 2.015274 & $0.944406-0.882592$ & $\mathrm{H} \quad 2.482983$ & 0.091360 & 0.000000 \\
\hline $\mathrm{H}$ & -1.711852 & $0.938177 \quad 1.292965$ & H $\quad-2.482983$ & -0.091360 & 0.000000 \\
\hline $\mathrm{H}$ & -1.711852 & $0.938177-1.292965$ & $\mathrm{H} \quad 0.218014$ & 2.184418 & 2.151768 \\
\hline $\mathrm{H}$ & -0.178168 & $0.949285-2.155075$ & H $\quad 0.218014$ & 2.184418 & -2.151768 \\
\hline $\mathrm{H}$ & -0.178168 & $0.949285 \quad 2.155075$ & $\mathrm{H} \quad-0.218014$ & -2.184418 & 2.151768 \\
\hline $\mathrm{C}$ & 0.765847 & $-3.036772 \quad 1.248021$ & H $\quad-0.218014$ & -2.184418 & -2.151768 \\
\hline $\mathrm{C}$ & 0.765847 & $-3.036772-1.248021$ & $\mathrm{H} \quad-1.983480$ & 2.185269 & -0.879393 \\
\hline $\mathrm{C}$ & -1.398459 & -3.0352350 .000000 & $\mathrm{H} \quad-1.983480$ & 2.185269 & 0.879393 \\
\hline $\mathrm{C}$ & 0.043573 & $-3.532210 \quad 0.000000$ & H 1.983480 & -2.185269 & -0.879393 \\
\hline $\mathrm{C}$ & 0.765847 & $-1.502189-1.252125$ & $\mathrm{H} \quad 1.983480$ & -2.185269 & 0.879393 \\
\hline $\mathrm{C}$ & 0.765847 & $-1.502189 \quad 1.252125$ & H 1.743858 & 2.197044 & -1.255380 \\
\hline $\mathrm{C}$ & -1.402117 & $-1.500841 \quad 0.000000$ & H 1.743858 & 2.197044 & 1.255380 \\
\hline $\mathrm{H}$ & 1.791124 & $-3.427002 \quad 1.254063$ & H $\quad-1.743858$ & -2.197044 & -1.255380 \\
\hline $\mathrm{H}$ & 1.791124 & $-3.427002-1.254063$ & $\mathrm{H} \quad-1.743858$ & -2.197044 & 1.255380 \\
\hline $\mathrm{H}$ & -1.917497 & $-3.426317 \quad 0.883879$ & C -0.722079 & -1.804804 & 1.254201 \\
\hline $\mathrm{H}$ & -1.917497 & $-3.426317 \quad-0.883879$ & C $\quad-0.722079$ & -1.804804 & -1.254201 \\
\hline $\mathrm{H}$ & 0.260780 & $-3.427158 \quad-2.140166$ & C $\quad 0.722079$ & 1.804804 & 1.254201 \\
\hline $\mathrm{H}$ & 0.260780 & $-3.427158 \quad 2.140166$ & C 0.722079 & 1.804804 & -1.254201 \\
\hline $\mathrm{C}$ & 1.474003 & $0.578198 \quad 0.000000$ & C 1.454746 & -1.802940 & 0.000000 \\
\hline $\mathrm{C}$ & -0.678941 & $0.571466 \quad 1.253994$ & C -1.454746 & 1.802940 & 0.000000 \\
\hline $\mathrm{C}$ & -0.678941 & $0.571466-1.253994$ & C 0.011679 & -2.299169 & 0.000000 \\
\hline $\mathrm{C}$ & -0.678089 & $-0.960652 \quad 1.250951$ & C -0.011679 & 2.299169 & 0.000000 \\
\hline $\mathrm{C}$ & -0.678089 & $-0.960652-1.250951$ & C 1.445271 & -0.267614 & 0.000000 \\
\hline $\mathrm{C}$ & 1.481098 & $-0.955383 \quad 0.000000$ & C -1.445271 & 0.267614 & 0.000000 \\
\hline $\mathrm{F}$ & 0.042384 & $-4.931955 \quad 0.000000$ & C $\quad-0.722079$ & -0.271713 & 1.251535 \\
\hline $\mathrm{H}$ & -1.197597 & $-1.322092 \quad 2.150008$ & C $\quad-0.722079$ & -0.271713 & -1.251535 \\
\hline $\mathrm{H}$ & -1.197597 & $-1.322092-2.150008$ & C 0.722079 & 0.271713 & 1.251535 \\
\hline $\mathrm{H}$ & 2.522855 & $-1.306530 \quad 0.000000$ & C $\quad 0.722079$ & 0.271713 & -1.251535 \\
\hline $\mathrm{C}$ & 0.035166 & $1.130553 \quad 0.000000$ & $\mathrm{~N} \quad-0.035830$ & -3.820932 & 0.000000 \\
\hline $\mathrm{C}$ & -0.018679 & $2.657956 \quad 0.000000$ & $\mathrm{~N} \quad 0.035830$ & 3.820932 & 0.000000 \\
\hline $\mathrm{C}$ & 1.129334 & $3.458663 \quad 0.000000$ & O $\quad-1.147014$ & -4.334005 & 0.000000 \\
\hline $\mathrm{C}$ & 1.043069 & $4.851479 \quad 0.000000$ & O 1.147014 & 4.334005 & 0.000000 \\
\hline $\mathrm{C}$ & -0.197474 & $5.480554 \quad 0.000000$ & O 1.019548 & -4.437089 & 0.000000 \\
\hline $\mathrm{C}$ & -1.260847 & $3.312491 \quad 0.000000$ & O $\quad-1.019548$ & 4.437089 & 0.000000 \\
\hline $\mathrm{C}$ & -1.353020 & $4.700483 \quad 0.000000$ & & & \\
\hline $\mathrm{H}$ & 2.112942 & $3.001071 \quad 0.000000$ & & & \\
\hline $\mathrm{H}$ & 1.954439 & $5.443606 \quad 0.000000$ & & & \\
\hline $\mathrm{H}$ & -0.265761 & $\begin{array}{ll}6.564767 & 0.000000\end{array}$ & & & \\
\hline $\mathrm{H}$ & -2.178441 & $2.729829 \quad 0.000000$ & & & \\
\hline & -2.331208 & $5.174169 \quad 0.000000$ & & & \\
\hline \multicolumn{3}{|c|}{$\mathrm{C}_{15} \mathrm{H}_{21} \mathrm{NO}_{2}$ RB3PW91\6-31G(d,p) E=-789.15688 } & \multicolumn{3}{|c|}{$\mathrm{C}_{14} \mathrm{H}_{19} \mathrm{NO}_{2}$ RB3PW91\6-31G(d,p) E=-749.85162 } \\
\hline $\mathrm{H}$ & -0.936328 & $-0.964342 \quad 2.150234$ & $\mathrm{H} \quad-1.206235$ & -0.864128 & 2.150680 \\
\hline $\mathrm{H}$ & -0.936328 & $-0.964342-2.150234$ & H $\quad-1.206235$ & -0.864128 & -2.150680 \\
\hline $\mathrm{H}$ & 0.372628 & $2.521632 \quad 0.000000$ & $\mathrm{H} \quad 0.098986$ & 2.624082 & 0.000000 \\
\hline $\mathrm{H}$ & -2.382876 & $1.144211 \quad 2.153441$ & $\mathrm{H} \quad-2.653491$ & 1.244997 & 2.157262 \\
\hline $\mathrm{H}$ & -2.382876 & $1.144211-2.153441$ & $\mathrm{H} \quad-2.653491$ & 1.244997 & -2.157262 \\
\hline $\mathrm{H}$ & -3.156770 & $-0.917372 \quad-0.881414$ & $\mathrm{H} \quad-3.427024$ & -0.823851 & -0.881599 \\
\hline $\mathrm{H}$ & -3.156770 & $-0.917372 \quad 0.881414$ & H $\quad-3.427024$ & -0.823851 & 0.881599 \\
\hline $\mathrm{H}$ & -1.846855 & $2.574407-1.272640$ & H $\quad-2.118912$ & 2.675769 & -1.275664 \\
\hline $\mathrm{H}$ & -1.846855 & $2.574407 \quad 1.272640$ & H $\quad-2.118912$ & 2.675769 & 1.275664 \\
\hline $\mathrm{C}$ & 1.014406 & $-1.143363 \quad 1.254028$ & C 0.744442 & -1.040539 & 1.254028 \\
\hline $\mathrm{C}$ & 1.014406 & $-1.143363-1.254028$ & C 0.744442 & -1.040539 & -1.254028 \\
\hline $\mathrm{C}$ & 1.781680 & $0.892168 \quad 0.000000$ & C 1.509371 & 0.995931 & 0.000000 \\
\hline $\mathrm{C}$ & 1.737099 & $-0.632535 \quad 0.000000$ & C 1.466132 & -0.528714 & 0.000000 \\
\hline $\mathrm{C}$ & 0.340520 & $1.423460 \quad 0.000000$ & C 0.067668 & 1.525891 & 0.000000 \\
\hline $\mathrm{C}$ & -0.421082 & $-0.601885 \quad 1.249951$ & C $\quad-0.691780$ & -0.500844 & 1.250269 \\
\hline $\mathrm{C}$ & -0.421082 & $-0.601885 \quad-1.249951$ & C $\quad-0.691780$ & -0.500844 & -1.250269 \\
\hline $\mathrm{H}$ & 1.547824 & $-0.804239 \quad 2.151195$ & H 1.277458 & -0.700988 & 2.151313 \\
\hline $\mathrm{H}$ & 1.547824 & $-0.804239 \quad-2.151195$ & $\mathrm{H} \quad 1.277458$ & -0.700988 & -2.151313 \\
\hline $\mathrm{H}$ & 1.022251 & $-2.238006-1.255606$ & $\mathrm{H} \quad 0.753465$ & -2.135229 & -1.255658 \\
\hline $\mathrm{H}$ & 1.022251 & $-2.238006 \quad 1.255606$ & $\mathrm{H} \quad 0.753465$ & -2.135229 & 1.255658 \\
\hline $\mathrm{H}$ & 2.326073 & $1.251105 \quad-0.879933$ & $\mathrm{H} \quad 2.053393$ & 1.355536 & -0.879947 \\
\hline $\mathrm{H}$ & 2.326073 & $\begin{array}{ll}1.251105 & 0.879933\end{array}$ & $\mathrm{H} \quad 2.053393$ & 1.355536 & 0.879947 \\
\hline $\mathrm{C}$ & -1.857582 & $1.475889 \quad 1.246877$ & C -2.129446 & 1.578015 & 1.251436 \\
\hline
\end{tabular}

URL: http://mc.manuscriptcentral.com/tandf/tmph 
2

3

4

5

6

7

8

9

10

11

12

13

14

15

16

17

18

19

20

21

22

23

24

25

26

27

28

29

30

31

32

33

34

35

36

37

38

39

40

41

42

43

44

45

46

47

48

49

50

51

52

53

54

55

56

57

58

59

60

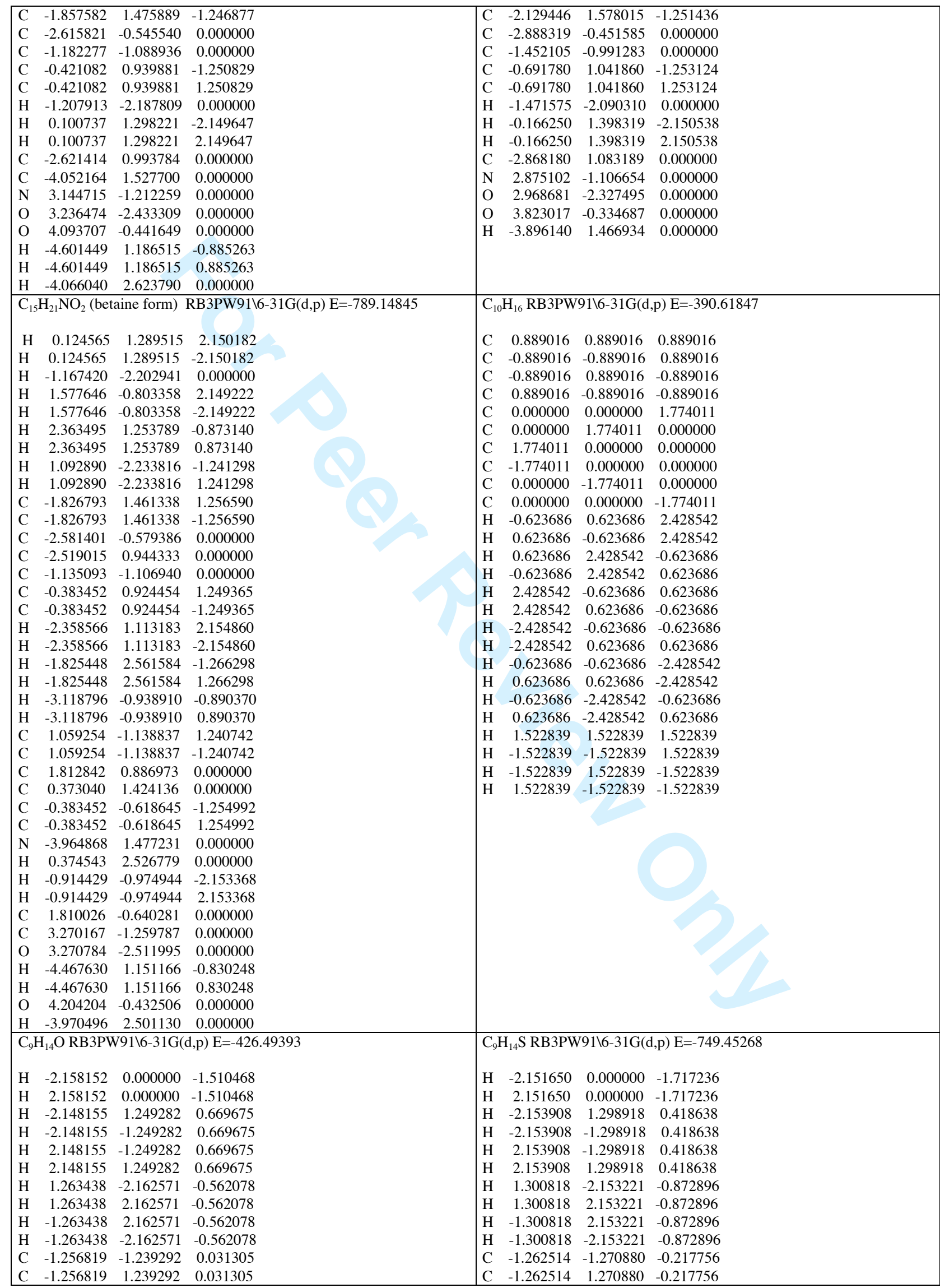

URL: http://mc.manuscriptcentral.com/tandf/tmph 
2

3

4

5

6

7

8

9

10

11

12

13

14

15

16

17

18

19

20

21

22

23

24

25

26

27

28

29

30

31

32

33

34

35

36

37

38

39

40

41

42

43

44

45

46

47

48

49

50

51

52

53

54

55

56

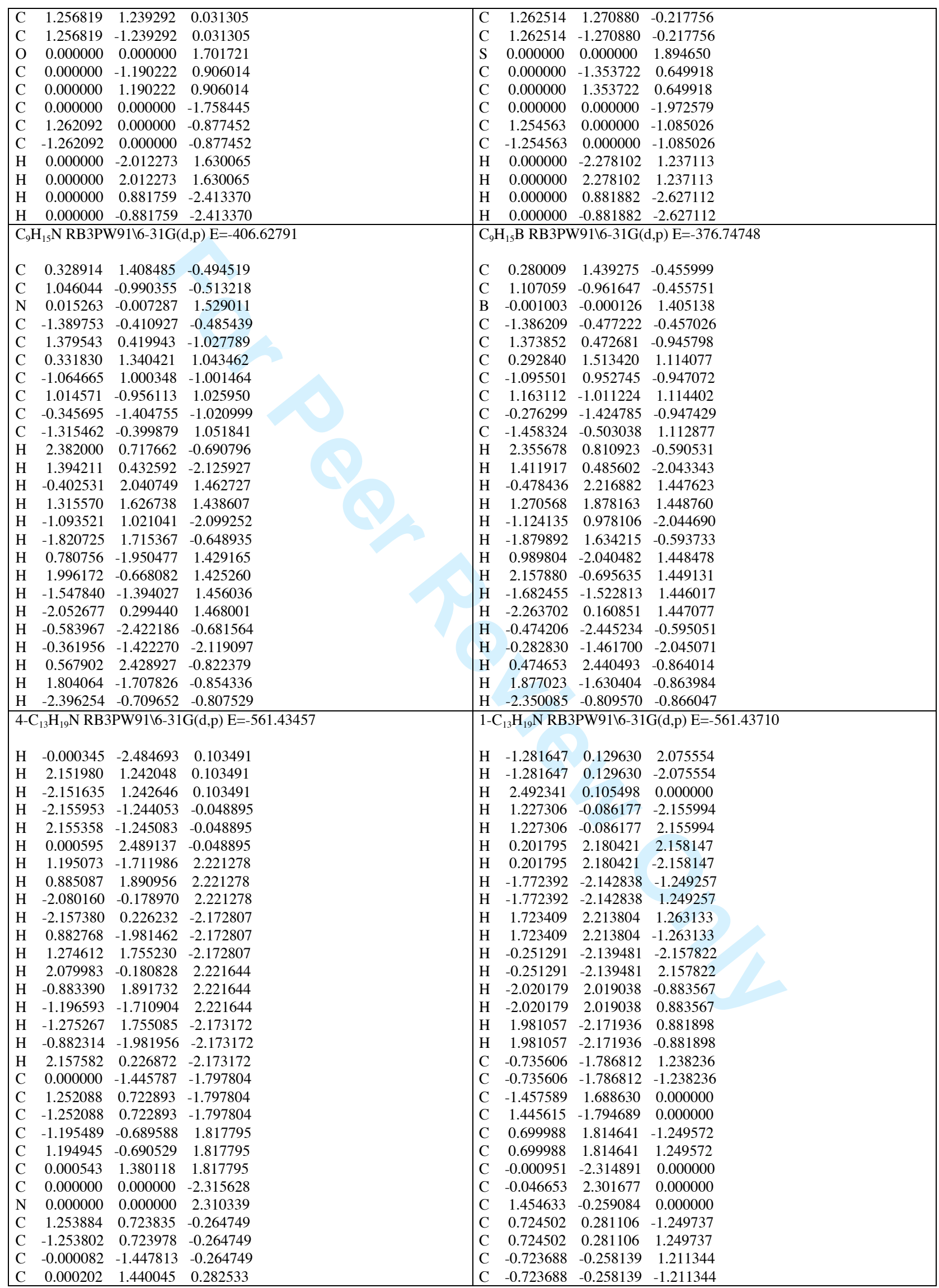

URL: http://mc.manuscriptcentral.com/tandf/tmph 
2

3

4

5

6

7

8

9

10

11

12

13

14

15

16

17

18

19

20

21

22

23

24

25

26

27

28

29

30

31

32

33

34

35

36

37

38

39

40

41

42

43

44

45

46

47

48

49

50

51

52

53

54

55

56

57

58

59

60

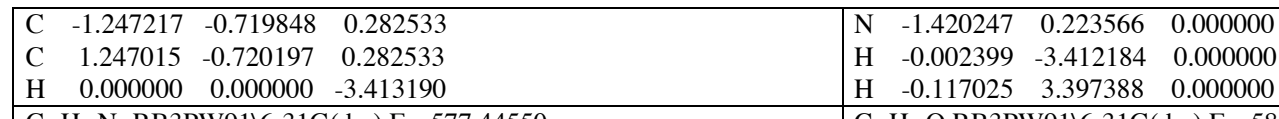

\begin{tabular}{|l|l}
\hline $\mathrm{C}_{12} \mathrm{H}_{18} \mathrm{~N}_{2}$ RB3PW9116-31G(d,p) E=-577.44550 & $\mathrm{C}_{13} \mathrm{H}_{18} \mathrm{O}$ RB3PW9116-31G(d,p) E=-581.30087
\end{tabular}

$\begin{array}{llll}\mathrm{H} & 0.000000 & 2.490862 & 0.055193\end{array}$

H $\quad-2.157150 \quad 1.245431-0.055193$

H $\quad-2.157150 \quad-1.245431 \quad 0.055193$

$\begin{array}{llll}\mathrm{H} & 2.157150 & 1.245431 & -0.055193\end{array}$

H $\quad 2.157150 \quad-1.245431 \quad 0.055193$

$\begin{array}{llll}\mathrm{H} & 0.000000 & -2.490862 & -0.055193\end{array}$

$\mathrm{H} \quad-1.195922 \quad 1.711602 \quad 2.215365$

$\begin{array}{lllll}\mathrm{H} & -0.884330 & 1.891500 & -2.215365\end{array}$

$\mathrm{H} \quad-0.884330-1.891500 \quad 2.215365$

$\begin{array}{llll}\mathrm{H} & 2.080252 & -0.179898 & -2.215365\end{array}$

$\begin{array}{llll}\mathrm{H} & 2.080252 & 0.179898 & 2.215365\end{array}$

H $\quad-1.195922-1.711602-2.215365$

H $\quad 0.884330 \quad-1.891500 \quad 2.215365$

H $\quad 1.195922-1.711602 \quad-2.215365$

H $\quad 1.195922 \quad 1.711602 \quad 2.215365$

$\mathrm{H} \quad-2.080252 \quad-0.179898 \quad-2.215365$

$\begin{array}{llll}\mathrm{H} & -2.080252 & 0.179898 & 2.215365\end{array}$

H $\quad 0.884330 \quad 1.891500-2.215365$

$\begin{array}{llll}\text { C } & 0.000000 & 1.380391 & -1.811617\end{array}$

$\begin{array}{llll}\text { C } & -1.195454 & 0.690196 & 1.811617\end{array}$

$\begin{array}{llll}\text { C } & -1.195454 & -0.690196 & -1.811617\end{array}$

C $\quad 1.195454 \quad 0.690196 \quad 1.811617$

$\begin{array}{llll}\text { C } & 1.195454 & -0.690196 & -1.811617\end{array}$

$\begin{array}{llll}\text { C } & 0.000000 & -1.380391 & 1.811617\end{array}$

$\begin{array}{llll}\mathrm{N} & 0.000000 & 0.000000 & -2.301748\end{array}$

$\begin{array}{llll}\mathrm{N} & 0.000000 & 0.000000 & 2.301748\end{array}$

$\begin{array}{llll}\text { C } & -1.248590 & -0.720874 & -0.277176\end{array}$

$\begin{array}{llll}\text { C } & 1.248590 & 0.720874 & 0.277176\end{array}$

$\begin{array}{llll}\text { C } & 1.248590 & -0.720874 & -0.277176\end{array}$

$\begin{array}{llll}\text { C } & 0.000000 & -1.441747 & 0.277176\end{array}$

$\begin{array}{llll}\text { C } & 0.000000 & 1.441747 & -0.277176\end{array}$

$\begin{array}{llll}\text { C } & -1.248590 & 0.720874 & 0.277176\end{array}$

$\begin{array}{llll}\mathrm{H} & -1.188393 & 0.384606 & 2.155061\end{array}$

$\begin{array}{lllll}\mathrm{H} & -1.188393 & 0.384606 & -2.155061\end{array}$

H $1.208498 \quad-0.340351 \quad 2.140154$

$\begin{array}{llll}\mathrm{H} & 1.208498 & -0.340351 & -2.140154\end{array}$

$\begin{array}{llll}\mathrm{H} & 2.316567 & -0.873025 & 0.000000\end{array}$

$\begin{array}{llll}\mathrm{H} & -2.273044 & 1.011250 & 0.000000\end{array}$

$\begin{array}{llll}\mathrm{H} & -2.628733 & -1.293463 & 0.882083\end{array}$

$\begin{array}{lllll}\mathrm{H} & -2.628733 & -1.293463 & -0.882083\end{array}$

$\begin{array}{llll}\mathrm{H} & 0.858776 & -2.654070 & 1.262482\end{array}$

H $\quad 0.858776-2.654070 \quad-1.262482$

$\begin{array}{llll}\mathrm{H} & -0.756609 & 2.701993 & 1.257968\end{array}$

$\begin{array}{llll}\mathrm{H} & -0.756609 & 2.701993 & -1.257968\end{array}$

H $\quad-0.557836 \quad-2.105895 \quad-2.157889$

$\begin{array}{llll}\mathrm{H} & -0.557836 & -2.105895 & 2.157889\end{array}$

$\begin{array}{llll}\mathrm{H} & 0.647368 & 2.090588 & -2.146381\end{array}$

$\begin{array}{llll}\mathrm{H} & 0.647368 & 2.090588 & 2.146381\end{array}$

$\begin{array}{llll}\text { C } & 0.031028 & 1.937327 & 1.252531\end{array}$

$\begin{array}{llll}\text { C } & 0.031028 & 1.937327 & -1.252531\end{array}$

$\begin{array}{llll}\text { C } & 0.031028 & -1.931918 & 1.247226\end{array}$

$\begin{array}{llll}\text { C } & 0.031028 & -1.931918 & -1.247226\end{array}$

$\begin{array}{llll}\text { O } & 1.934080 & 1.103029 & 0.000000\end{array}$

$\begin{array}{llll}\text { C } & -1.993043 & -1.137629 & 0.000000\end{array}$

$\begin{array}{llll}\text { C } & 0.895917 & 2.087932 & 0.000000\end{array}$

$\begin{array}{llll}\text { C } & -0.836911 & -2.148751 & 0.000000\end{array}$

$\begin{array}{llll}\text { C } & -0.570891 & 0.528625 & -1.256873\end{array}$

$\begin{array}{llll}\text { C } & -0.570891 & 0.528625 & 1.256873\end{array}$

$\begin{array}{llll}\text { C } & 0.577470 & -0.499569 & -1.255804\end{array}$

$\begin{array}{llll}\text { C } & 0.577470 & -0.499569 & 1.255804\end{array}$

$\begin{array}{llll}\text { C } & 1.425865 & -0.232438 & 0.000000\end{array}$

$\begin{array}{llll}\text { C } & -1.439683 & 0.293752 & 0.000000\end{array}$

$\begin{array}{llll}\mathrm{H} & 1.419345 & 3.050037 & 0.000000\end{array}$

$\begin{array}{llll}\mathrm{H} & -1.238144 & -3.170095 & 0.000000\end{array}$

$\mathrm{C}_{13} \mathrm{H}_{19} \mathrm{~B}$ RB3PW91\6-31G(d,p) E=-531.55185

H $\quad-1.335907 \quad-0.272623 \quad 2.148662$

H $\quad-1.335907 \quad-0.272623 \quad-2.148662$

$\begin{array}{llll}\mathrm{H} & 1.142690 & -0.048098 & 2.146760\end{array}$

$\begin{array}{llll}\mathrm{H} & 1.142690 & -0.048098 & -2.146760\end{array}$

H $\quad 2.390689-0.304764 \quad 0.000000$

$\begin{array}{llll}\mathrm{H} & -2.589742 & -0.084669 & 0.000000\end{array}$

$\begin{array}{llll}\mathrm{H} & -2.074228 & -2.354888 & 0.882094\end{array}$

H $\quad-2.074228 \quad-2.354888-0.882094$

$\begin{array}{llll}\mathrm{H} & 1.655557 & -2.329737 & 1.287367\end{array}$

$\begin{array}{lllll}\mathrm{H} & 1.655557 & -2.329737 & -1.287367\end{array}$

$\begin{array}{llll}\mathrm{H} & -1.955864 & 1.948474 & 1.304973\end{array}$

$\mathrm{H} \quad-1.955864 \quad 1.948474 \quad-1.304973$

H $\quad 0.121151-2.329470 \quad-2.158061$

$\mathrm{H} \quad 0.121151 \quad-2.329470 \quad 2.158061$

$\begin{array}{llll}\mathrm{H} & -0.407020 & 2.044827 & -2.152059\end{array}$

$\begin{array}{llll}\mathrm{H} & -0.407020 & 2.044827 & 2.152059\end{array}$

$\begin{array}{llll}\text { C } & -0.900487 & 1.641671 & 1.260812\end{array}$

$\begin{array}{llll}\text { C } & -0.900487 & 1.641671 & -1.260812\end{array}$

$\begin{array}{llll}\text { C } & 0.622505 & -1.958291 & 1.254079\end{array}$

$\begin{array}{llll}\text { C } & 0.622505 & -1.958291 & -1.254079\end{array}$

$\begin{array}{llll}\text { S } & 1.542535 & 1.901409 & 0.000000\end{array}$

$\begin{array}{llll}\text { C } & -1.540286 & -1.976314 & 0.000000\end{array}$

$\begin{array}{llll}\text { C } & -0.263574 & 2.234498 & 0.000000\end{array}$

$\begin{array}{llll}\text { C } & -0.091977 & -2.482710 & 0.000000\end{array}$

$\begin{array}{llll}\text { C } & -0.830913 & 0.107743 & -1.249019\end{array}$

$\begin{array}{llll}\text { C } & -0.830913 & 0.107743 & 1.249019\end{array}$

$\begin{array}{llll}\text { C } & 0.622505 & -0.420377 & -1.255426\end{array}$

$\begin{array}{llll}\text { C } & 0.622505 & -0.420377 & 1.255426\end{array}$

$\begin{array}{llll}\text { C } & 1.362318 & 0.077506 & 0.000000\end{array}$

$\begin{array}{llll}\text { C } & -1.550183 & -0.443746 & 0.000000\end{array}$

$\begin{array}{llll}\mathrm{H} & -0.354896 & 3.325710 & 0.000000\end{array}$

$\begin{array}{llll}\mathrm{H} & 2.150985 & 1.242374 & -0.166218\end{array}$

$\begin{array}{lllll}\mathrm{H} & 0.000435 & -2.483995 & -0.166218\end{array}$

$\begin{array}{llll}\mathrm{H} & -2.151420 & 1.241621 & -0.166218\end{array}$

$\begin{array}{llll}\mathrm{H} & 0.000223 & 2.483717 & 0.079987\end{array}$

$\begin{array}{llll}\mathrm{H} & 2.150850 & -1.242052 & 0.079987\end{array}$

$\begin{array}{llll}\mathrm{H} & -2.151074 & -1.241665 & 0.079987\end{array}$

$\begin{array}{llll}\mathrm{H} & 2.250854 & -0.268493 & -2.220969\end{array}$

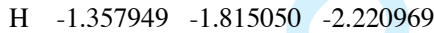

$\begin{array}{lllll}\mathrm{H} & -0.892905 & 2.083543 & -2.220969\end{array}$

$\begin{array}{llll}\mathrm{H} & -1.274689 & 1.755297 & 2.111810\end{array}$

$\begin{array}{llll}\mathrm{H} & 2.157476 & 0.226265 & 2.111810\end{array}$

$\begin{array}{llll}\mathrm{H} & -0.882787 & -1.981561 & 2.111810\end{array}$

$\begin{array}{llll}\mathrm{H} & 1.361881 & -1.811824 & -2.223288\end{array}$

H $\quad-2.250026 \quad-0.273511 \quad-2.223288$

$\begin{array}{llll}\mathrm{H} & 0.888145 & 2.085335 & -2.223288\end{array}$

$\begin{array}{llll}\mathrm{H} & -2.157171 & 0.228778 & 2.113337\end{array}$

$\begin{array}{llll}\mathrm{H} & 1.276713 & 1.753775 & 2.113337\end{array}$

H $\quad 0.880457 \quad-1.982554 \quad 2.113337$

$\begin{array}{llll}\text { C } & 1.252492 & 0.722505 & 1.736667\end{array}$

$\begin{array}{llll}\text { C } & -0.000538 & -1.445942 & 1.736667\end{array}$

$\begin{array}{llll}\text { C } & -1.251954 & 0.723437 & 1.736667\end{array}$

$\begin{array}{llll}\text { C } & -0.001447 & 1.538071 & -1.890945\end{array}$

$\begin{array}{llll}\text { C } & 1.332732 & -0.767782 & -1.890945\end{array}$

$\begin{array}{llll}\text { C } & -1.331285 & -0.770289 & -1.890945\end{array}$

$\begin{array}{llll}\text { C } & 0.000000 & 0.000000 & 2.253928\end{array}$

$\begin{array}{llll}\text { B } & 0.000000 & 0.000000 & -2.186192\end{array}$

$\begin{array}{llll}\text { C } & 0.000310 & -1.447908 & 0.200558\end{array}$

$\begin{array}{llll}\text { C } & -1.254080 & 0.723685 & 0.200558\end{array}$

$\begin{array}{llll}\text { C } & 1.253770 & 0.724222 & 0.200558\end{array}$

$\begin{array}{llll}\text { C } & -1.265312 & -0.730528 & -0.325617\end{array}$

$\begin{array}{llll}\text { C } & 0.000000 & 1.461056 & -0.325617\end{array}$ 
2

\begin{tabular}{|c|c|c|c|c|c|c|c|}
\hline $\mathrm{H}$ & -0.080496 & -3.580149 & 0.000000 & $\begin{array}{l}\mathrm{C} \\
\mathrm{H}\end{array}$ & $\begin{array}{l}1.265312 \\
0.000000\end{array}$ & $\begin{array}{r}-0.730528 \\
0.000000\end{array}$ & $\begin{array}{r}-0.325617 \\
3.351497\end{array}$ \\
\hline \multicolumn{4}{|c|}{$4,9-\mathrm{C}_{12} \mathrm{H}_{18} \mathrm{~B}_{2}$ RB3PW91\6-31G(d,p) E=-517.67973 } & \multicolumn{4}{|c|}{$4,9-\mathrm{C}_{12} \mathrm{H}_{18} \mathrm{~B}_{\mathrm{N}} \mathrm{RB} 3 \mathrm{PW} 91 \backslash 6-31 \mathrm{G}(\mathrm{d}, \mathrm{p}) \mathrm{E}=-547.56273$} \\
\hline $\mathrm{H}$ & 0.000000 & 2.483829 & 0.153075 & $\mathrm{H}$ & 0.114962 & 1.278171 & 2.136374 \\
\hline $\mathrm{H}$ & -2.151059 & 1.241914 & -0.153075 & $\mathrm{H}$ & -0.086461 & -1.208983 & 2.170883 \\
\hline $\mathrm{H}$ & -2.151059 & -1.241914 & 0.153075 & $\mathrm{H}$ & 0.118337 & -2.489407 & 0.038267 \\
\hline $\mathrm{H}$ & 2.151059 & 1.241914 & -0.153075 & $\mathrm{H}$ & -0.085644 & 2.485139 & -0.038284 \\
\hline $\mathrm{H}$ & 2.151059 & -1.241914 & 0.153075 & $\mathrm{H}$ & 0.118271 & 1.211936 & -2.174451 \\
\hline $\mathrm{H}$ & 0.000000 & -2.483829 & -0.153075 & $\mathrm{H}$ & -0.083801 & -1.274973 & -2.133239 \\
\hline $\mathrm{H}$ & -1.361934 & 1.813842 & 2.153576 & $\mathrm{H}$ & 2.213839 & -0.235565 & 2.255633 \\
\hline $\mathrm{H}$ & -0.889866 & 2.086390 & -2.153576 & $\mathrm{H}$ & -2.157081 & 0.214170 & 2.076086 \\
\hline $\mathrm{H}$ & -0.889866 & -2.086390 & 2.153576 & $\mathrm{H}$ & 2.215549 & -1.834747 & -1.330941 \\
\hline $\mathrm{H}$ & 2.251800 & -0.272548 & -2.153576 & $\mathrm{H}$ & -2.155030 & 1.691657 & -1.224411 \\
\hline $\mathrm{H}$ & 2.251800 & 0.272548 & 2.153576 & $\mathrm{H}$ & 2.215252 & 2.070863 & -0.922143 \\
\hline $\mathrm{H}$ & -1.361934 & -1.813842 & -2.153576 & $\mathrm{H}$ & -2.154919 & -1.908360 & -0.852042 \\
\hline $\mathrm{H}$ & 0.889866 & -2.086390 & 2.153576 & $\mathrm{H}$ & 2.215832 & -0.306564 & -2.246088 \\
\hline $\mathrm{H}$ & 1.361934 & -1.813842 & -2.153576 & $\mathrm{H}$ & -2.153065 & 0.148276 & -2.083642 \\
\hline $\mathrm{H}$ & 1.361934 & 1.813842 & 2.153576 & $\mathrm{H}$ & 2.214759 & 2.098503 & 0.859199 \\
\hline $\mathrm{H}$ & -2.251800 & -0.272548 & -2.153576 & $\mathrm{H}$ & -2.152747 & -1.877845 & 0.913970 \\
\hline $\mathrm{H}$ & -2.251800 & 0.272548 & 2.153576 & $\mathrm{H}$ & 2.214796 & -1.791932 & 1.389086 \\
\hline $\mathrm{H}$ & 0.889866 & 2.086390 & -2.153576 & $\mathrm{H}$ & -2.156585 & 1.729381 & 1.168266 \\
\hline $\mathrm{C}$ & 0.000000 & 1.540430 & -1.820602 & $\mathrm{C}$ & -1.752649 & 0.708700 & 1.183312 \\
\hline $\mathrm{C}$ & -1.334051 & 0.770215 & 1.820602 & $\mathrm{C}$ & 1.884161 & -0.747927 & 1.344475 \\
\hline $\mathrm{C}$ & -1.334051 & -0.770215 & -1.820602 & $\mathrm{C}$ & -1.750388 & -1.380879 & 0.021580 \\
\hline $\mathrm{C}$ & 1.334051 & 0.770215 & 1.820602 & $\mathrm{C}$ & 1.884859 & 1.538064 & -0.022993 \\
\hline $\mathrm{C}$ & 1.334051 & -0.770215 & -1.820602 & $\mathrm{C}$ & -1.750195 & 0.671401 & -1.206628 \\
\hline $\mathrm{C}$ & 0.000000 & -1.540430 & 1.820602 & $\mathrm{C}$ & 1.885477 & -0.789614 & -1.319338 \\
\hline B & 0.000000 & 0.000000 & -2.116158 & $\mathrm{~N}$ & -2.241846 & -0.000711 & -0.001089 \\
\hline B & 0.000000 & 0.000000 & 2.116158 & B & 2.180840 & 0.000151 & 0.000738 \\
\hline $\mathrm{C}$ & -1.267029 & -0.731520 & -0.255058 & $\mathrm{C}$ & -0.212289 & -1.441149 & 0.021756 \\
\hline $\mathrm{C}$ & 1.267029 & 0.731520 & 0.255058 & $\mathrm{C}$ & 0.319711 & 1.462099 & -0.022071 \\
\hline $\mathrm{C}$ & 1.267029 & -0.731520 & -0.255058 & $\mathrm{C}$ & -0.212289 & 0.701477 & -1.258744 \\
\hline $\mathrm{C}$ & 0.000000 & -1.463040 & 0.255058 & $\mathrm{C}$ & 0.320904 & -0.750026 & -1.254651 \\
\hline $\mathrm{C}$ & 0.000000 & 1.463040 & -0.255058 & $\mathrm{C}$ & -0.214484 & 0.739794 & 1.236389 \\
\hline $\mathrm{C}$ & -1.267029 & 0.731520 & 0.255058 & $\mathrm{C}$ & 0.319259 & -0.711190 & 1.277146 \\
\hline \multicolumn{4}{|c|}{ 1,6-C12H18BN RB3PW91\6-31G(d,p) E=-547.56671 } & \multicolumn{4}{|c|}{ C18H24 RB3PW91\6-31G(d,p) E=-700.23078 } \\
\hline $\mathrm{H}$ & 1.241362 & -0.053483 & 2.240387 & $\mathrm{C}$ & 1.250555 & 1.274410 & 0.695332 \\
\hline $\mathrm{H}$ & 1.241362 & -0.053483 & -2.240387 & $\mathrm{C}$ & -1.250555 & 1.274410 & 0.695332 \\
\hline $\mathrm{H}$ & -1.245257 & 0.149724 & 2.069746 & $\mathrm{C}$ & -1.250555 & -1.274410 & 0.695332 \\
\hline $\mathrm{H}$ & -1.245257 & 0.149724 & -2.069746 & $\mathrm{C}$ & 1.250555 & -1.274410 & 0.695332 \\
\hline $\mathrm{H}$ & -0.212287 & -2.126927 & 2.156759 & $\mathrm{C}$ & 0.000000 & 0.000000 & 2.476548 \\
\hline $\mathrm{H}$ & -0.212287 & -2.126927 & -2.156759 & $\mathrm{C}$ & 0.000000 & 1.254099 & 1.597543 \\
\hline $\mathrm{H}$ & 0.232228 & 2.235662 & 2.154029 & $\mathrm{C}$ & 0.000000 & -1.254099 & 1.597543 \\
\hline $\mathrm{H}$ & 0.232228 & 2.235662 & -2.154029 & $\mathrm{C}$ & 1.249379 & 0.000000 & -0.170742 \\
\hline $\mathrm{H}$ & -1.746728 & -2.116586 & -1.275157 & $\mathrm{C}$ & -1.249379 & 0.000000 & -0.170742 \\
\hline $\mathrm{H}$ & -1.746728 & -2.116586 & 1.275157 & $\mathrm{C}$ & 1.251795 & -2.524304 & -0.193173 \\
\hline $\mathrm{H}$ & 1.767128 & 2.268246 & -1.293004 & $\mathrm{C}$ & -1.251795 & -2.524304 & -0.193173 \\
\hline $\mathrm{H}$ & 1.767128 & 2.268246 & 1.293004 & $\mathrm{C}$ & -1.251795 & 2.524304 & -0.193173 \\
\hline $\mathrm{H}$ & 1.996164 & -2.353413 & -0.889774 & $\mathrm{C}$ & 1.251795 & 2.524304 & -0.193173 \\
\hline $\mathrm{H}$ & 1.996164 & -2.353413 & 0.889774 & $\mathrm{H}$ & 0.881867 & -1.261850 & -2.614905 \\
\hline $\mathrm{H}$ & -1.956057 & 2.037783 & -0.883838 & $\mathrm{H}$ & -0.881867 & -1.261850 & -2.614905 \\
\hline $\mathrm{H}$ & -1.956057 & 2.037783 & 0.883838 & $\mathrm{H}$ & -0.881867 & 1.261850 & -2.614905 \\
\hline $\mathrm{C}$ & 0.753425 & 1.847723 & 1.265319 & $\mathrm{H}$ & 0.881867 & 1.261850 & -2.614905 \\
\hline $\mathrm{C}$ & 0.753425 & 1.847723 & -1.265319 & $\mathrm{C}$ & 0.000000 & 0.000000 & -1.091810 \\
\hline $\mathrm{C}$ & -0.705225 & -1.776280 & 1.241858 & $\mathrm{H}$ & -0.881853 & 0.000000 & 3.132148 \\
\hline $\mathrm{C}$ & -0.705225 & -1.776280 & -1.241858 & $\mathrm{H}$ & 0.881853 & 0.000000 & 3.132148 \\
\hline $\mathrm{C}$ & -1.393154 & 1.709508 & 0.000000 & $\mathrm{H}$ & 0.000000 & -2.151495 & 2.233319 \\
\hline $\mathrm{C}$ & 1.510521 & -1.936117 & 0.000000 & $\mathrm{H}$ & 0.000000 & 2.151495 & 2.233319 \\
\hline $\mathrm{C}$ & 0.018138 & 2.316259 & 0.000000 & $\mathrm{C}$ & 0.000000 & -2.526660 & -1.081910 \\
\hline $\mathrm{C}$ & -0.005225 & -2.347315 & 0.000000 & $\mathrm{C}$ & 0.000000 & 2.526660 & -1.081910 \\
\hline $\mathrm{N}$ & -1.354359 & 0.244531 & 0.000000 & $\mathrm{C}$ & 0.000000 & -1.266781 & -1.957825 \\
\hline B & 1.331645 & -0.378224 & 0.000000 & $\mathrm{C}$ & 0.000000 & 1.266781 & -1.957825 \\
\hline $\mathrm{C}$ & 0.805016 & 0.313368 & -1.302657 & $\mathrm{H}$ & -1.275454 & -3.428644 & 0.429881 \\
\hline $\mathrm{C}$ & 0.805016 & 0.313368 & 1.302657 & $\mathrm{H}$ & 1.275454 & -3.428644 & 0.429881 \\
\hline $\mathrm{C}$ & -0.683873 & -0.245325 & -1.210402 & $\mathrm{H}$ & 1.275454 & 3.428644 & 0.429881 \\
\hline $\mathrm{C}$ & -0.683873 & -0.245325 & 1.210402 & $\mathrm{H}$ & -1.275454 & 3.428644 & 0.429881 \\
\hline $\mathrm{H}$ & -0.059352 & 3.411731 & 0.000000 & $\mathrm{H}$ & -2.157076 & -2.541838 & -0.815111 \\
\hline $\mathrm{H}$ & -0.085250 & -3.442167 & 0.000000 & $\mathrm{H}$ & 2.157076 & -2.541838 & -0.815111 \\
\hline & & & & $\mathrm{H}$ & 2.157076 & 2.541838 & -0.815111 \\
\hline
\end{tabular}




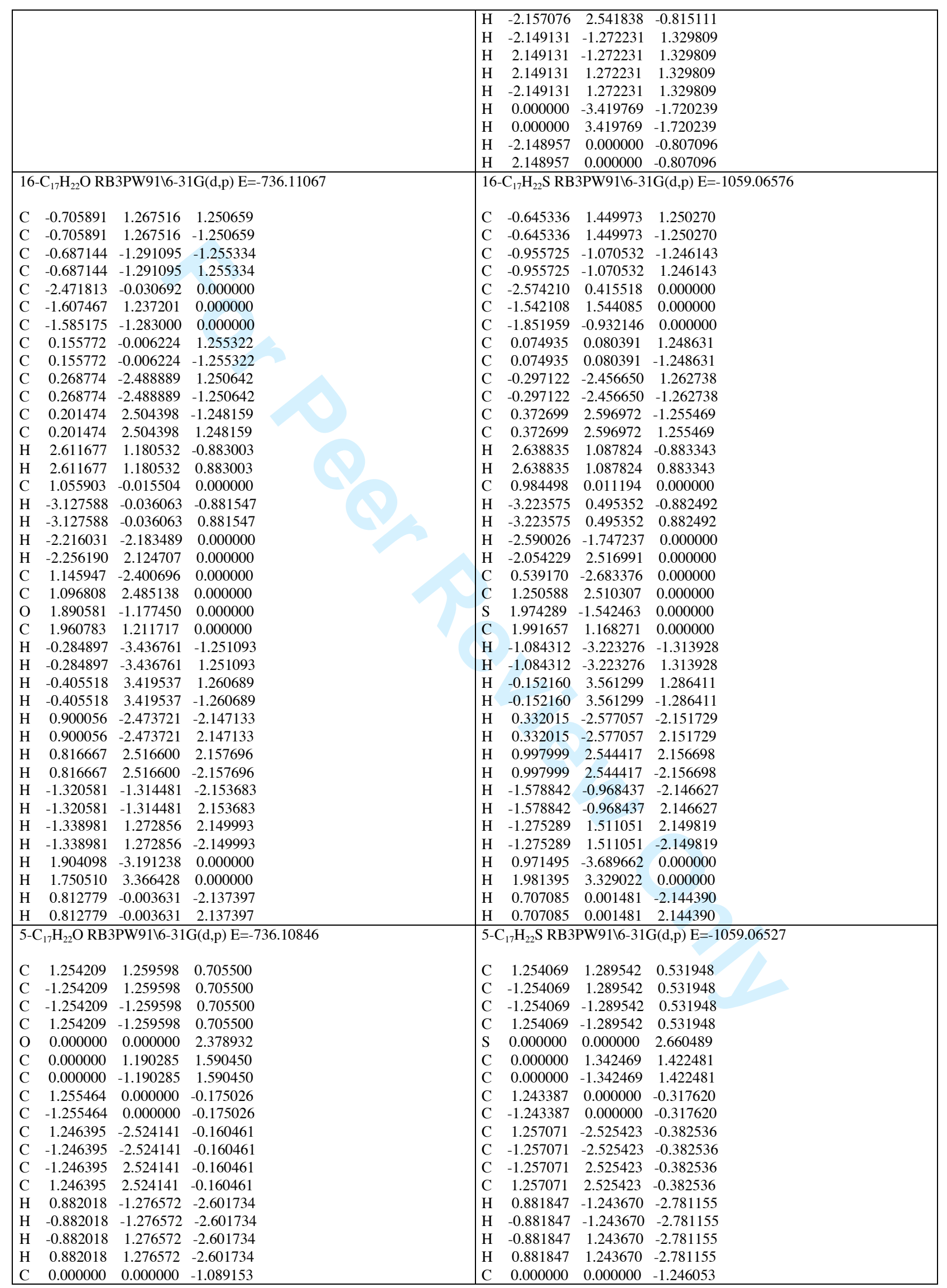


2

3

4

5

6

7

8

9

10

11

12

13

14

15

16

17

18

19

20

21

22

23

24

25

26

27

28

29

30

31

32

33

34

35

36

37

38

39

40

41

42

43

44

45

46

47

48

49

50

51

52

53

54

55

56

57

58

59

60

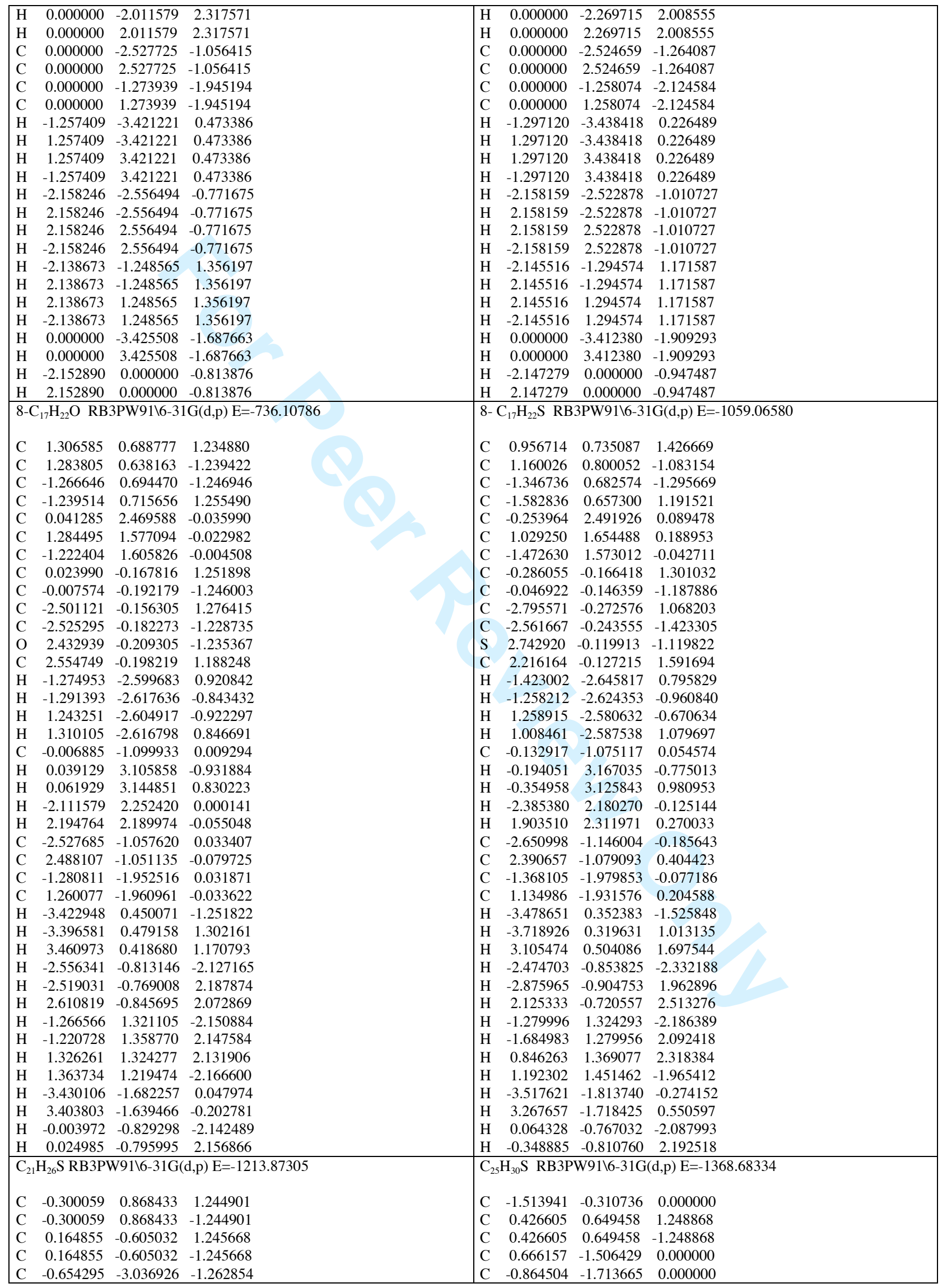

URL: http://mc.manuscriptcentral.com/tandf/tmph 


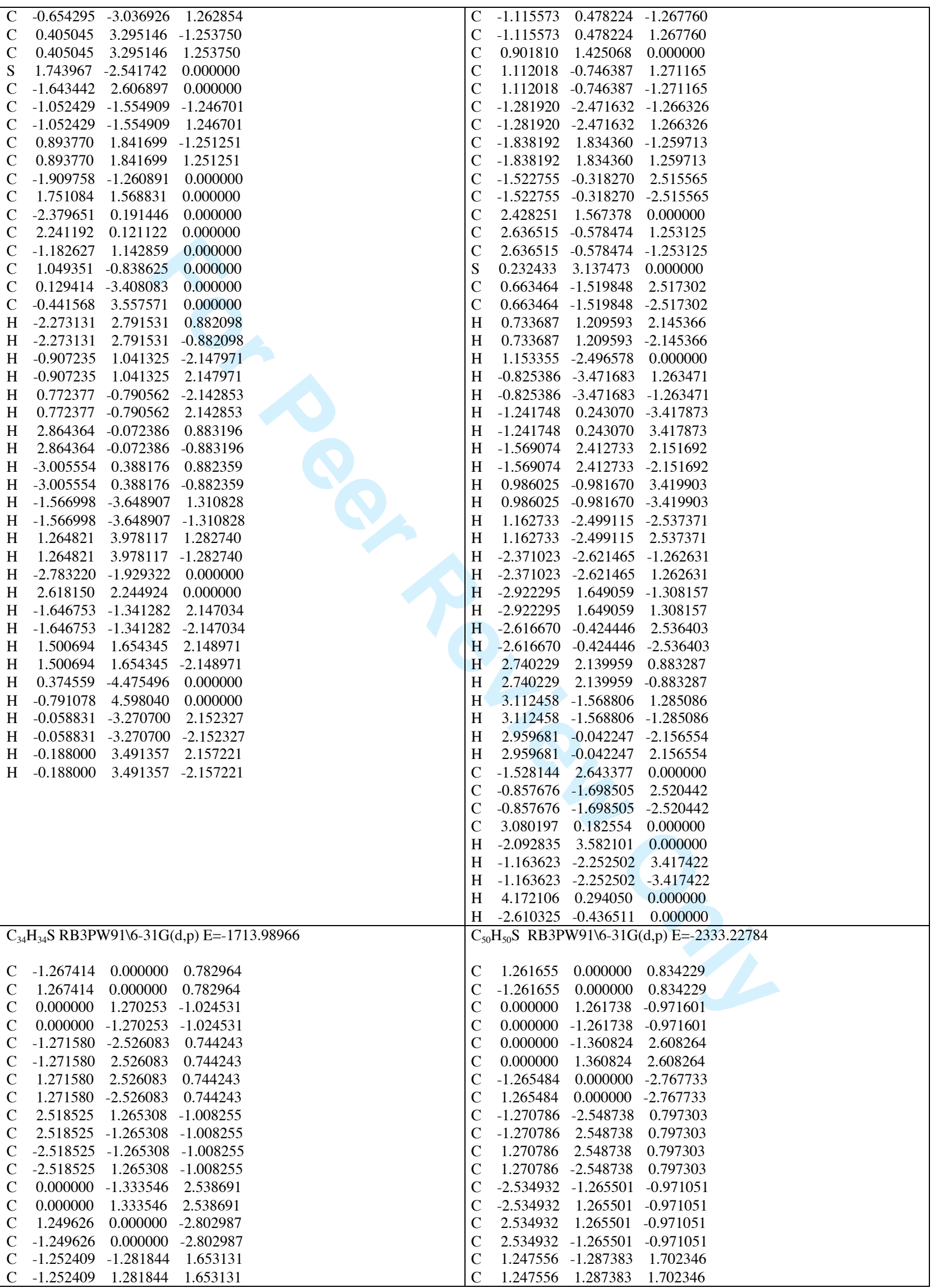


$\begin{array}{llll}\mathrm{C} & -1.247556 & 1.287383 & 1.702346 \\ \mathrm{C} & -1.247556 & -1.287383 & 1.702346\end{array}$

$\begin{array}{llll}\text { C } & -1.247556 & -1.287383 & 1.702346\end{array}$

$\begin{array}{llll}\text { C } & -1.264657 & 1.240970 & -1.850271\end{array}$

$\begin{array}{llll}\text { C } & -1.264657 & -1.240970 & -1.850271\end{array}$

$\begin{array}{llll}\text { C } & 1.264657 & -1.240970 & -1.850271\end{array}$

$\begin{array}{llll}\text { C } & 1.264657 & 1.240970 & -1.850271\end{array}$

$\begin{array}{llll}\text { C } & 0.000000 & -2.511637 & -0.079727\end{array}$

$\begin{array}{llll}\text { C } & 0.000000 & 2.511637 & -0.079727\end{array}$

$\begin{array}{llll}\text { C } & -2.508396 & 0.000000 & -0.082409\end{array}$

$\begin{array}{llll}\text { C } & 2.508396 & 0.000000 & -0.082409\end{array}$

$\begin{array}{lllll}\text { C } & 0.000000 & 0.000000 & -0.067084\end{array}$

$\begin{array}{llll}\text { C } & -2.514230 & -2.512452 & -0.090225\end{array}$

$\begin{array}{llll}\text { C } & -2.514230 & 2.512452 & -0.090225\end{array}$

$\begin{array}{llll}\text { C } & 2.514230 & 2.512452 & -0.090225\end{array}$

$\begin{array}{llll}\text { C } & 2.514230 & -2.512452 & -0.090225\end{array}$

$\begin{array}{llll}\text { S } & 0.000000 & 0.000000 & 3.820789\end{array}$

$\begin{array}{llll}\text { C } & 0.000000 & 0.000000 & -3.628150\end{array}$

$\begin{array}{llll}\mathrm{H} & -3.409450 & 0.000000 & 0.554571\end{array}$

$\begin{array}{llll}\mathrm{H} & 3.409450 & 0.000000 & 0.554571\end{array}$

$\begin{array}{lllll}\mathrm{H} & 0.000000 & -3.402958 & -0.730580\end{array}$

$\begin{array}{llll}\mathrm{H} & 0.000000 & 3.402958 & -0.730580\end{array}$

$\begin{array}{llll}\text { H } & 1.261006 & -2.144789 & -2.483922\end{array}$

$\begin{array}{llll}\mathrm{H} & 1.261006 & 2.144789 & -2.483922\end{array}$

$\begin{array}{llll}\mathrm{H} & -1.261006 & 2.144789 & -2.483922\end{array}$

$\begin{array}{llll}\mathrm{H} & -1.261006 & -2.144789 & -2.483922\end{array}$

$\begin{array}{llll}\mathrm{H} & -2.143285 & 1.291032 & 2.342486\end{array}$

$\begin{array}{llll}\mathrm{H} & -2.143285 & -1.291032 & 2.342486\end{array}$

$\begin{array}{llll}\mathrm{H} & 2.143285 & -1.291032 & 2.342486\end{array}$

H $\quad 2.143285 \quad 1.291032 \quad 2.342486$

$\begin{array}{llll}\text { C } & 1.255056 & -3.823549 & 1.651150\end{array}$

$\begin{array}{llll}\text { C } & 1.255056 & 3.823549 & 1.651150\end{array}$

$\begin{array}{llll}\text { C } & -1.255056 & 3.823549 & 1.651150\end{array}$

$\begin{array}{llll}\text { C } & -1.255056 & -3.823549 & 1.651150\end{array}$

$\begin{array}{llll}\text { C } & -3.781036 & 1.247290 & -1.866137\end{array}$

$\begin{array}{llll}\text { C } & -3.781036 & -1.247290 & -1.866137\end{array}$

$\begin{array}{llll}\text { C } & 3.781036 & -1.247290 & -1.866137\end{array}$

$\begin{array}{llll}\text { C } & 3.781036 & 1.247290 & -1.866137\end{array}$

$\begin{array}{llll}\text { C } & 0.000000 & -2.648092 & 3.443064\end{array}$

$\begin{array}{llll}\text { C } & 0.000000 & 2.648092 & 3.443064\end{array}$

$\begin{array}{llll}\text { C } & -2.530117 & 0.000000 & -3.635521\end{array}$

$\begin{array}{llll}\text { C } & 2.530117 & 0.000000 & -3.635521\end{array}$

$\begin{array}{llll}\mathrm{H} & -2.542610 & -3.412458 & -0.722760\end{array}$

$\begin{array}{lllll}\mathrm{H} & -2.542610 & 3.412458 & -0.722760\end{array}$

$\begin{array}{llll}\mathrm{H} & 2.542610 & 3.412458 & -0.722760\end{array}$

$\begin{array}{llll}\mathrm{H} & 2.542610 & -3.412458 & -0.722760\end{array}$

$\begin{array}{llll}\mathrm{H} & -3.417177 & -2.540775 & 0.537947\end{array}$

$\begin{array}{llll}\mathrm{H} & -3.417177 & 2.540775 & 0.537947\end{array}$

$\begin{array}{llll}\mathrm{H} & 3.417177 & 2.540775 & 0.537947\end{array}$

$\begin{array}{lllll}\mathrm{H} & 3.417177 & -2.540775 & 0.537947\end{array}$

H $\quad 0.000000 \quad-0.882153 \quad-4.286242$

$\begin{array}{llll}\mathrm{H} & 0.000000 & 0.882153 & -4.286242\end{array}$

$\begin{array}{llll}\mathrm{H} & -2.156134 & -3.860026 & 2.279548\end{array}$

$\begin{array}{llll}\mathrm{H} & -2.156134 & 3.860026 & 2.279548\end{array}$

$\begin{array}{llll}\mathrm{H} & 2.156134 & 3.860026 & 2.279548\end{array}$

$\begin{array}{llll}\mathrm{H} & 2.156134 & -3.860026 & 2.279548\end{array}$

H $\quad-3.799108-2.156328-2.484075$

$\begin{array}{llll}\mathrm{H} & -3.799108 & 2.156328 & -2.484075\end{array}$

H $3.799108 \quad 2.156328-2.484075$

$\begin{array}{llll}\mathrm{H} & 3.799108 & -2.156328 & -2.484075\end{array}$

$\begin{array}{llll}\mathrm{H} & -1.293831 & -4.700080 & 0.989206\end{array}$

$\begin{array}{llll}\mathrm{H} & -1.293831 & 4.700080 & 0.989206\end{array}$

H $\quad 1.293831 \quad 4.700080 \quad 0.989206$

H $\quad 1.293831 \quad-4.700080 \quad 0.989206$

H $\quad-4.684032-1.271724-1.239735$

H $\quad-4.684032 \quad 1.271724 \quad-1.239735$

H $4.684032 \quad 1.271724 \quad-1.239735$

H $\quad 4.684032-1.271724 \quad-1.239735$

$\mathrm{H} \quad-0.882992 \quad-2.654109 \quad 4.095824$

$\begin{array}{llll}\mathrm{H} & -0.882992 & 2.654109 & 4.095824\end{array}$

$\begin{array}{llll}\mathrm{H} & 0.882992 & 2.654109 & 4.095824\end{array}$

$\begin{array}{llll}\mathrm{H} & 0.882992 & -2.654109 & 4.095824\end{array}$ 


\section{Page 43 of 43}

Molecular Physics

1

S30

2

3

4

5

6

7

8

9

10

11

12

13

14

15

16

17

18

19

20

21

22

23

24

25

26

27

28

29

30

31

32

33

34

35

36

37

38

39

40

41

42

43

44

45

46

47

48

49

50

51

52

53

54

55

56

57

58

59

60

URL: http://mc.manuscriptcentral.com/tandf/tmph 